\title{
A Survey of Standards for the U.S. Fiber/Textile/Apparel Industry
}

Craig G. Pawlak

U.S. DEPARTMENT OF COMMERCE Technology Administration National Institute of Standards and Technology

Manufacturing Systems Integration Division Gaithersburg, MD 20899

QC

100

.056

N0.5823

1996 



\section{A Survey of Standards for the U.S. Fiber/Textile/Apparel Industry}

Craig G. Pawlak

U.S. DEPARTMENT OF COMMERCE

Technology Administration

National Institute of Standards

and Technology

Manufacturing Systems Integration Division

Gaithersburg, MD 20899

April 1996

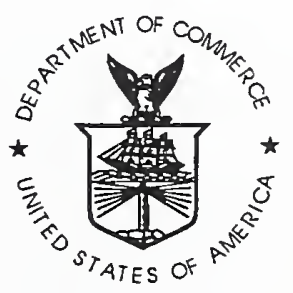

U.S. DEPARTMENT OF COMMERCE

Michael Kantor, Secretary

TECHNOLOGY ADMINISTRATION

Mary L. Good, Under Secretary for Technology

NATIONAL INSTITUTE OF STANDARDS

AND TECHNOLOGY

Arati Prabhakar, Director 


\section{DISCLAIMER}

No approval or endorsement of any commercial product, organization, or company by the National Institute of Standards and Technology is intended or implied. Certain commercial equipment, instruments, or materials may be identified in this report in order to facilitate understanding. Such identification does not imply recommendation or endorsement by the National Institute of Standards and Technology, nor does it imply that the materials or equipment identified are necessarily the best available for the purpose.

This publication was prepared by United States Government employees as part of their official duties and is, therefore, a work of the U.S. Government and not subject to copyright. 


\title{
A SURVEY of STANDARDS for the U.S. FIBER/TEXTILE/APPAREL INDUSTRY
}

\author{
Craig G. Pawlak \\ Manufacturing Systems Integration Division \\ Manufacturing Engineering Laboratory \\ National Institute of Standards and Technology \\ Gaithersburg, MD
}

\begin{abstract}
This report documents a survey of standards relevant to the U.S. Fiber/Textile/Apparel (FTA) industry. The standards are discussed in four main groups-integration standards, test methods, quality standards, and standard reference data and materials. The Appendix of the report lists the titles of all standards found, grouped together by the organization responsible for them. Those organizations are also listed along with contact information for them. The report attempts to bring together useful information concerning FTA standards as a starting point to support the industry in intelligently planning future standards' development efforts.
\end{abstract}

\section{KEYWORDS}

apparel, fiber, integration, quality, specifications, standards, test methods, textile 


\section{ACKNOWLEDGMENTS}

This work is supported by the government initiative on High Performance Computing and Communications (HPCC), which is primarily being supported through the Systems Integration for Manufacturing Applications (SIMA) initiative. The work is being done by the Manufacturing Systems Integration Division of the Manufacturing Engineering Laboratory of the National Institute of Standards and Technology.

I would like to thank my supervisor, Jeane Ford, for her support of this project, and Howard Moncarz for his editing and other help. 


\section{TABLE OF CONTENTS}

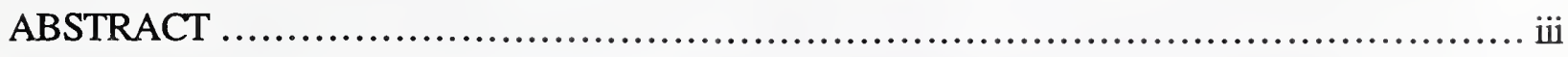

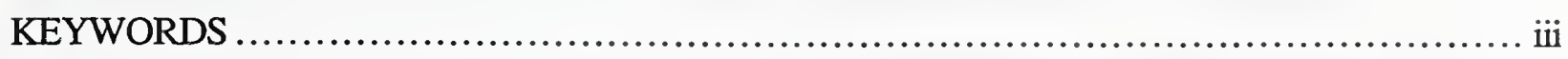

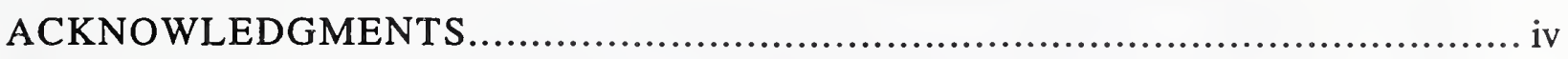

TABLE OF CONTENTS.....................................................................

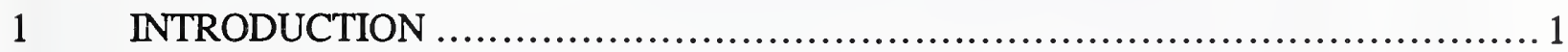

1.1 Purpose

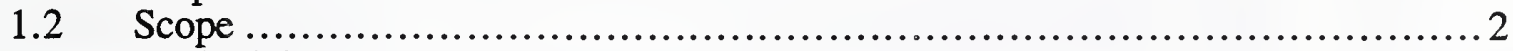

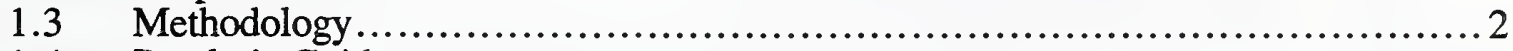

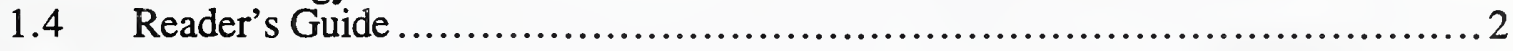

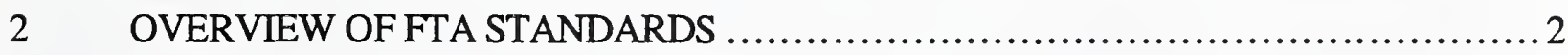

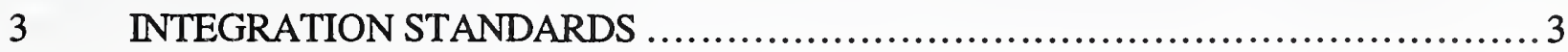

3.1 Business/EDI Transactions............................................................. 4

3.2 Manufacturing Automation ..................................................... 6

3.3 Product Data Exchange ....................................................... 7

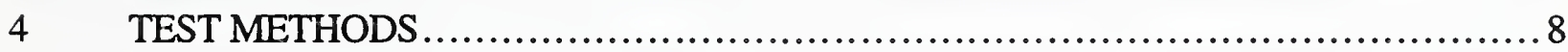

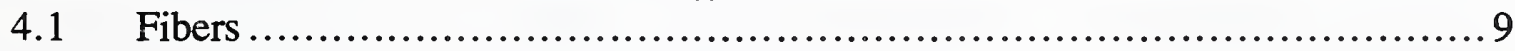

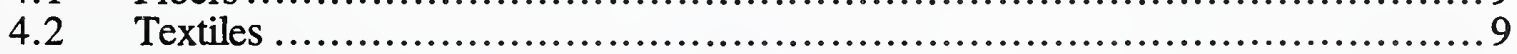

5 QUALITY STANDARDS............................................................. 10

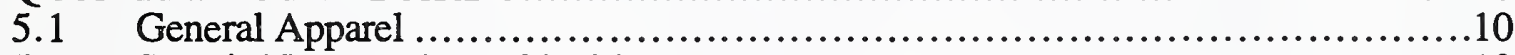

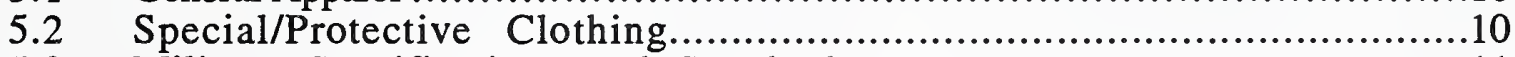

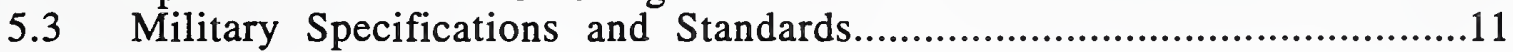

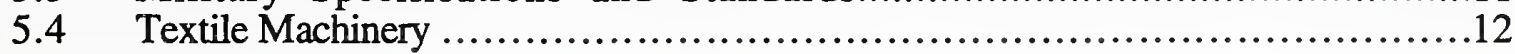

6 STANDARD REFERENCE INFORMATION .......................................13

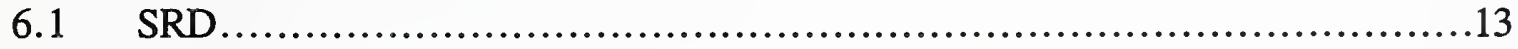

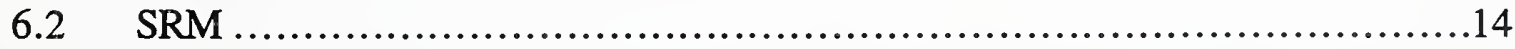

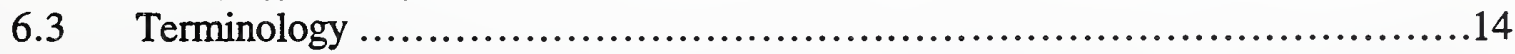

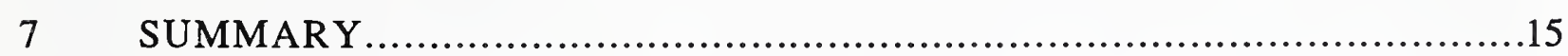




\section{APPENDICES}

A REFERENCES .............................................................. 17

B FTA STANDARDS ORGANIZATIONS ......................................... 19

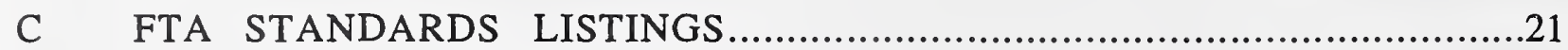

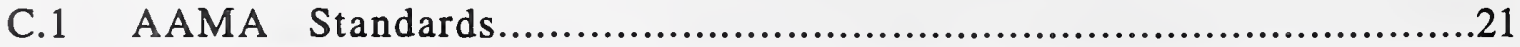

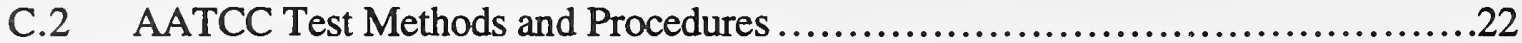

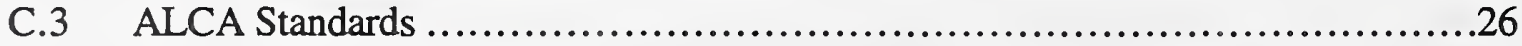

C. 4 ASTM Textile Standards ...................................................30

C.5 Government/Military Standards and Specifications .............................40

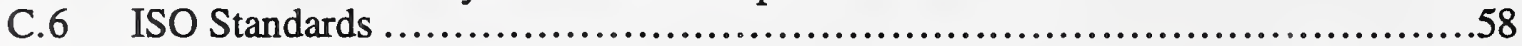

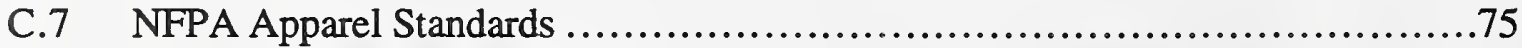

C.8 SAE AMS Textile Specifications ............................................76

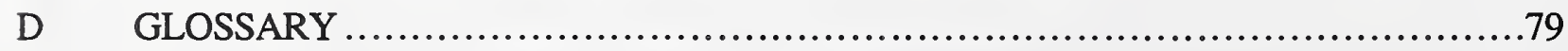

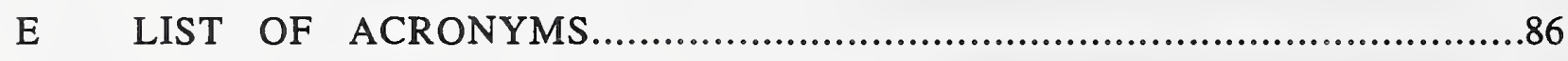

\section{FIGURE}

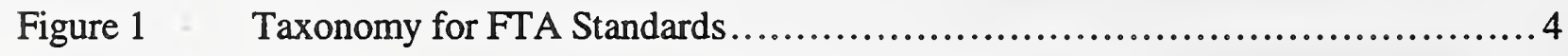

\section{TABLES}

Table 1

Table 2

Table 3

Cotton Staple Qualities...........................................................80

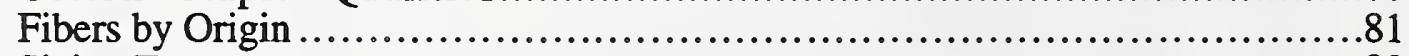

Sizing Types 


\title{
A SURVEY of STANDARDS for the U.S. FIBER/TEXTILE/APPAREL INDUSTRY
}

\author{
Craig G. Pawlak \\ Manufacturing Systems Integration Division \\ Manufacturing Engineering Laboratory \\ National Institute of Standards and Technology \\ Gaithersburg, MD
}

\section{INTRODUCTION}

The Fiber/Textile/Apparel (or FTA) industry is one of the largest manufacturing industries in the United States. It employs over one and a half million people, accounting for ten percent of all jobs in the U.S. manufacturing sector. Apparel and textile products shipped each year are worth well over one hundred billion dollars. The success of the FTA industry in the United States is critical to the economic well-being of our country.

However, in the last decade, the FTA industry's domestic markets, which are key, have been seriously eroded by foreign imports. As a result, hundreds of thousands of jobs have been lost over the past ten years and new job opportunities have been missed as well.

The American Textile Partnership (AMTEX), initiated in mid-1992, is a collaboration of industry research consortia and academia working in conjunction with the U.S. Department of Energy (DOE) national laboratories, to provide assistance to the U.S. FTA industry to recover its domestic market share and enhance its global competitiveness. In June 1995, the National Institute of Standards and Technology (NIST) in the U.S. Department of Commerce (DoC) officially joined the AMTEX collaboration. The survey described in this report is the first effort undertaken by NIST in the AMTEX effort. The goal of the survey is to help identify the standards that apply to the entire FTA industry. 1

\subsection{Purpose}

The survey is intended to benefit the Demand Activated Manufacturing Architecture (DAMA) Project, one of the key AMTEX projects. The main goal of DAMA is to reduce the long cycle time that it takes for a product to ultimately work its way through the "apparel pipeline"-from fiber production to an apparel product on the retail shelf. The long cycle time costs the industry an estimated $\$ 25$ billion a year due to stockouts, inventory, and distressed pricing. The goal of DAMA is to greatly reduce that loss by improving the efficiency of information exchange throughout the pipeline and enabling effective action as a result of that information. Understanding what standards apply throughout the pipeline should be useful to that effort.

This paper reports on the results of that survey. It identifies standards related to the FTA industry, identifies and describes the organizations responsible for approving those standards, and directs the reader to the appropriate sources for further information.

\footnotetext{
${ }^{1}$ AMTEX identifies the FTA industry by the term "integrated textile complex," and has coined the acronym, "ITC."
} 


\subsection{Scope}

The survey covers both national and international standards and standards organizations involved with and relating to the fiber, textile, and apparel industries. This includes industry standards, which make up the majority of the standards found, as well as any specifications issued by the government (such as the MIL-series). This report focuses on standards that are currently in effect, although past standards and current work may be mentioned to provide additional background and understanding.

There are many products of the fiber and textile sectors of the FTA industry that do not go through the entire life-cycle apparel pipeline (from fiber to textile to apparel to customer). Standards relating to fiber products that do not end up as textiles, but are rather used for industrial purposes, are included within the scope of this survey. Also included are fiber products such as rope or webbing, which do not eventually become part of a garment. In addition, any products that are fabricated from fiber and textile products are included. However, the main focus of this survey concentrates on standards used in the apparel pipeline.

\subsection{Methodology}

A general search of standards for the industry was accomplished through database searches as well as the use of other reference material. The sources used are listed in Appendix A. The approach was centered on determining the standards organizations for different sectors of the industry. The standards organizations are listed in Appendix B. After identifying the organizations, lists of their standards were obtained. The listings are transcribed for use in Appendix C. For the purposes of discussing the standards in the text, they were organized into four main groups.

\subsection{Reader's Guide}

Section 2 provides an overview of the different groups for the standards that were found. Sections 3 through 6 describe each group of standards in greater detail. Section 7 concludes the main text of the paper with a brief summary.

Following the text are five appendices-A, B, C, D, and E. Appendix A contains a list of references that are referred to in the text as well as additional references that are useful for further information. Appendix B identifies standards' organizations relevant to the FTA industry with a brief description of each and contact information. Appendix $C$ lists the titles of the standards found over the course of this survey. The documents are grouped according to the standards organization responsible for each. Appendix D contains a glossary of terms related to the FTA industry. Appendix $\mathrm{E}$ is a brief list of acronyms used in the paper, intended for quick reference.

\section{OVERVIEW OF FTA STANDARDS}

Based on an examination of the types of FTA standards found (determined by looking at titles, content, and usage), standards were divided into four broad types. The standards are divided into integration standards, test methods, quality standards, and standard reference data and materials. Although divided for the purposes of discussion, standards listed in Appendix C are grouped by publishing organization.

The first group contains integration standards. These are standards which allow one system or activity to communicate with another. The types of communications may range from one machine 
communicating with another on a shop floor to one company ordering materials or products from a supplier. Integration standards are covered in more detail in Section 3.

The second, and largest, group of standards found contains test methods and procedures. These are methods for testing properties of anything from raw fibers, to yarns, to woven fabrics, or even the machinery used to make textiles. The standards themselves are arranged in the form of an experiment format, with sections on materials, procedures, and observations. Properties determined by this group of tests range from the tensile strength of raw cotton fiber to a fabric's ability to resist fading or running (colorfastness). More detail about test methods can be found in Section 4.

The third group is quality standards. These deal with more functional properties of a finished fabric or apparel product. Quality standards specify how to determine if certain products are suitable for the application intended. The specification might cover the protective ability of a fireproof jacket, or the stitch spacing of a dress. The bulk of these standards are military specifications for combat apparel, but there are many important standards which apply to other areas. Section 5 discusses quality standards in greater detail.

The remaining group of standards consists of standard reference materials (SRM), standard reference data (SRD), and terminology. An SRM is used to rate by direct comparison other data or materials for different applications. An SRD is a collection of numerical information accepted as accurate within a domain. For example, anthropometric data (body measurements of different types of people), is used by companies for apparel sizing (an example of SRD), and standard color or color change charts or samples are used in the apparel industry for direct comparison tests relating to colorfastness (an example of SRM). More detail can be found in Section 6.

Figure 1 (on page 4) shows the grouping used in this discussion. In the figure, the four broad groups of standards are each decomposed further to show the types of standards contained.

\section{INTEGRATION STANDARDS}

Integration is the process of unifying separate items, that is to make multiple objects (whether they be machines, computers, or entire sectors) act as if they were one unit. This is contingent upon accurate communication of ideas and information between the (different) parties involved. For this to occur, both parties must agree upon and use the same protocol, or "language." There are many accepted standards of communication for the multitudes of different interfaces existing in the FTA industry. These interfaces exist from one end of the FTA life cycle to the other, and the efficiency and effectiveness of the communication across these junctions has a major effect on the efficiency of the industry.

Since the area of integration is so important, special attention is needed here. Time delays between the different phases in the life cycle are due most directly to ineffective communication. These delays (manifested in the need to keep large inventory) are responsible for $\$ 25$ billion being lost annually. The loss occurs through markdowns, stockouts, and inventory maintenance. The other result of lack of integration is that the FTA industry as a whole cannot respond to demand directly, but rather must anticipate it, a less desirable situation.

This section will discuss integration standards that are in existence now, as well as describe some standards that are under development. Some of the most important work is still underway, so special attention will be given to these up-and-coming protocols. Division of the integration-related standards is done by what type of interface is affected. The three processes discussed are business transactions, automated manufacturing, and product data exchange. 


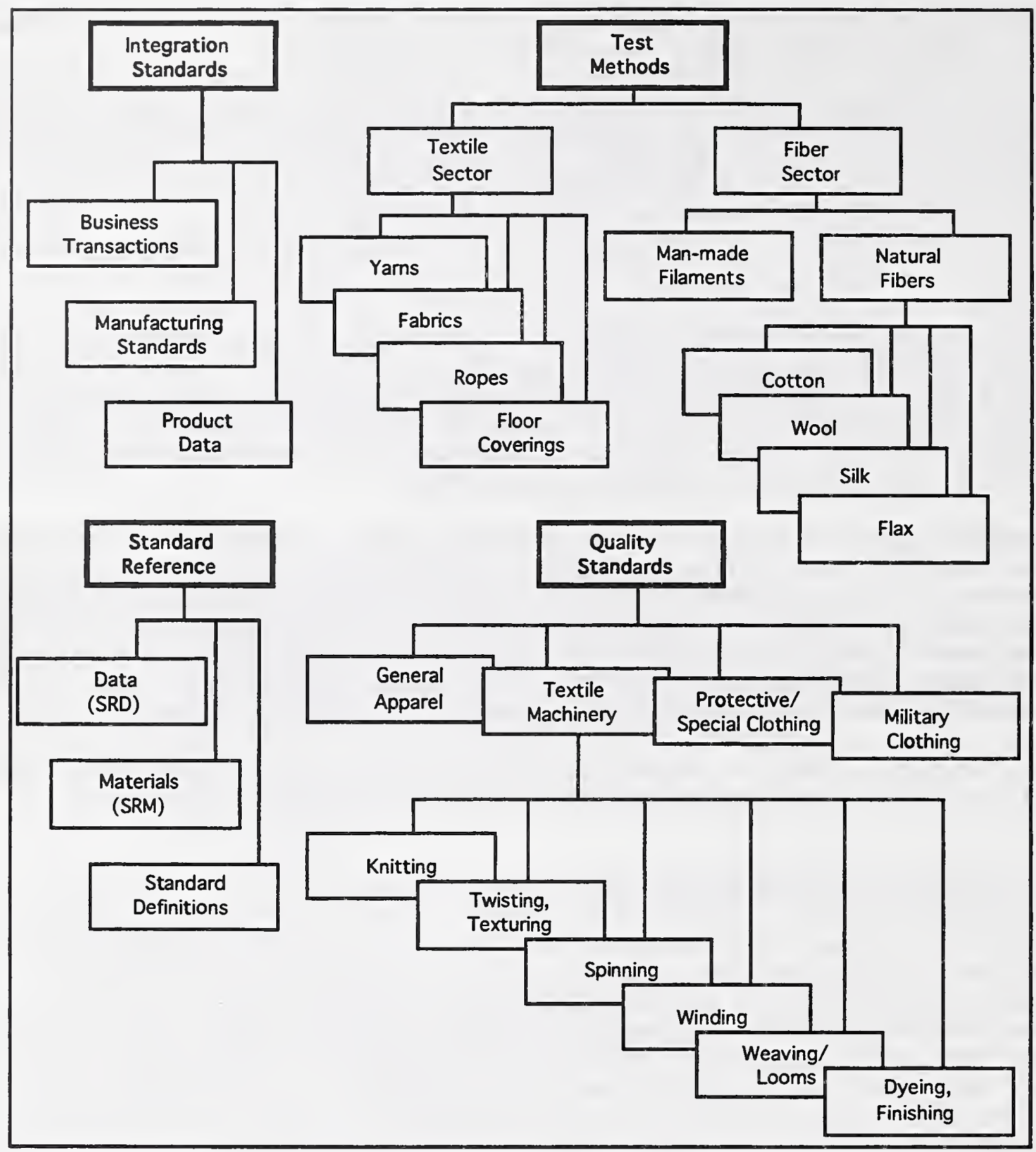

\section{Figure 1: Taxonomy for FTA Standards}

\subsection{Business/EDI Transactions}

Put simply, electronic data interchange (EDI) is the process of conducting business electronically, rather than by paper. Communication occurs between two computers, rather than between two people $^{2}$. This includes many different types of transactions, such as placing orders, transferring funds (payment), and confirming receipt of goods. Although the task of creating electronic protocols to replace all the different (paper) forms used in various kinds of businesses is daunting, the benefits in terms of efficiency, accuracy, and ability to trace make it more than worthwhile.

2 Shaw, p. 5. 1994. 
Because the information is transmitted rather than mailed, EDI is faster than the traditional paper method. Since the "forms" don't have to pass through as many different sets of hands, danger of an order being miswritten, misplaced, or permanently lost is almost completely eliminated. And lastly, electronic transmission allows one to trace the history of a form, a feature not always available with normal mail.

Although EDI began in the 1960s, standards development didn't begin until 1978, when the American National Standards Institute (ANSI) founded the Accredited Standards Committee (ASC) $\mathrm{X} 12^{3}$. This organization was chartered with the responsibility of creating transaction sets (protocol for a specific business exchange) for electronic commerce. X12 grew over time and has established over two hundred different transaction sets through more than a dozen subcommittees. Although these standards have been very widely used in North America, most industries have found it necessary or desirable to modify the basic transaction sets in different ways to better suit their business.

The international EC/EDI effort is known as the United Nations Electronic Data Interchange for Administration, Commerce, and Transport (UN/EDIFACT or just EDIFACT) standard. UN/EDIFACT came about with the merger of the original EDIFACT with the United Nations Trade Data Interchange (UN/TDI). This standard has often been seen as a competitor to X12, though in fact their methods of organization and design rules are quite similar ${ }^{4}$. By the very nature of EDI only one protocol can be used, unless the two are somehow made compatible. For this reason, ASC X12 announced a deadline for converting to the international standard. This was initially set for 1997, but was later extended to at least 1999, pending a poll of EDI users that will be conducted the same year as the original deadline ${ }^{5}$. As part of the EDIFACT initiative, the International Organization for Standardization's (ISO) Technical Committee (TC) 154 developed some syntax rules, which were first published in $1988^{6}$. They have since been revised.

In the FTA industry, EDI standards work can be divided into three domains: textile, apparel, and retail. The Fabric and Supplier Linkage Council (FASLINC) was established to adapt X12 standards to the specific needs of textile companies and their suppliers (in the fiber sector). This is accomplished mainly by inserting textile-specific codes into existing transaction sets, but a few new transaction sets were actually developed by FASLINC and approved by ASC X12. FASLINC as an entity was discontinued and its standards and responsibilities were transferred to the Apparel Textile Manufacturers Institute (ATMI).

The Textile Apparel Linkage Council (TALC) and the Sundries and Findings Linkage Council (SAFLINC) promote and develop electronic commerce standards for clothing manufacture. Founded in 1986, TALC is responsible for interactions between fabric suppliers and apparel companies. SAFLINC handles business with the suppliers of non-textile materials needed for garments, such as zippers and buttons. These two organizations were merged to form TALC/SAFLINC, which is now part of the American Apparel Manufacturers Association (AAMA).

There are several EDI standards used in the apparel-retail sector. The Uniform Communication Standards (UCS) began development in the early 1980's for use by the grocery industry, but has

3 McCarthy, p. 94. 1995.

4 Arnoff \& Hsing, p. 5. 1995.

5 Gaffin, p. 31. 1994.

ASC X12. 1995.

6 ISO 9735:1988 : "Electronic Data Interchange for Administration, Commerce and Transport (EDIFACT) Application level syntax rules (Amended and reprinted 1990)." Amendment 1 added in 1992. 
since been expanded in its scope and application ${ }^{7}$. It consists of about thirty transaction sets, as well as the stated protocol of using the public phone system with a modem speed of 4800 or 9600 baud. The Warehouse Information Network Standard (WINS) consists of seven transaction sets for that aspect of retail. The emerging standards for use by all types of merchandising industries is the Voluntary Interindustry Communication Standard (VICS). VICS is a subset of ANSI X12 pertaining specially to retail. The domains for UCS and VICS overlap. For more information on any of these standards, contact the Uniform Code Council at the address and number listed in Appendix B.

\subsection{Manufacturing Automation}

Much of the manufacturing of textiles is automated. Monitoring and control of the many different machines present on the shop floor can require a lot of people, in the worst case one per machine. Although most of the machines don't require constant monitoring or input, setting them up or changing a weaving pattern, for example, can take a lot of time. Even shutting down a machine often requires a long process, and can be dangerous if there are personnel in the wrong places on the shop floor. This is important because utility companies commonly offer textile manufacturers significant saving on their electricity if they can shut down power in a short span of time (this reduces the peak load and keeps the power company from switching to less efficient back-up generators).

Integrating the manufacturing process allows an entire shop floor to be run from the convenience (and safety) of one central control booth. This can only take place if all the machines are compatible with the controller and each other. Presently, companies making textile machinery use proprietary methods of storing and communicating information such as speed of a process or error warnings. As a result, these machines can only be integrated with others made by the same company - an inconvenience for textile manufacturers who may already have other equipment. If the makers of textile machinery adopted voluntary standards for shop floor data, CIM for textiles would be much easier to achieve.

One of the major proponents of computer-integrated manufacturing (CIM) for the FTA industry is ATMI. To aid in the development of voluntary standards, ATMI is working on a dictionary of data elements for control and monitoring of textile processes. The rationale is that in order to come up with a universal data set for a certain process, one must first identify all the different variables involved. This is being done in conjunction with ISO TC 72, and will be published in parts as ISO 10782. The first part covers spinning and related processes. At the present time, the dictionary contains over 100 variables that require attention, as well as definitions and a method of organization. It is currently in the draft stage and under committee review.

Also, the Apparel Research Committee (ARC) of AAMA has been developing standards related to CIM (as well as product data, which will be discussed in the next section), for apparel manufacturing. The first AAMA standard published and approved by ANSI is a modified version of Gerber Garment Technology, Inc.'s protocol for automated cutting machines ${ }^{8}$. A second standard of AAMA deals with pattern data interchange (PDI) ${ }^{9}$. The PDI standard also pertains to interfacing computer-aided design (CAD) systems with computer-aided manufacturing (CAM) systems. Work is in progress on a standard for NC stitching machines and a CIM architecture standard 10 .

\footnotetext{
7 Uniform Code Council, p. 2. 1994.

8 ANSI/AAMA-001-1992: "Standard for Numerically Controlled Cutting Machines."

9 ANSUAAMA-292-1993 : "Standard for Pattern Data Interchange - Data Format."

10 AAMA. 1995.
} 


\subsection{Product Data Exchange}

Product data includes information from every stage in the life cycle of a product. This extends from initial design through manufacturing, shipping, and even recycling of the product. A standard for product data has as its goal the accommodation of all the computer interfaces a product will encounter, thus integrating the life cycle. The benefits include independence from any particular software tools (such as a certain CAD system); continuity of data (same format of information can follow the product through the different stages of its life); and the ability to communicate a neutral data format between different departments, sectors, and even industries.

The international standard for development of total product data is called STEP (standard for the exchange of product model data). It is being developed in conjunction with ISO by TC 184/SC 4 . STEP is being published (in many parts) as ISO 10303 . Parts of STEP that have already finished the approval process include standards relating to drafting and design. At the present time, there are over forty more ISO 10303 parts in some stage of planning, development, or approval.

STEP is an open methodology and framework for the development of product data models and specifications. STEP uses a language for modeling information that is known as EXPRESS ${ }^{11}$. Within STEP, Application Protocols (APs) are created that specify the product information requirements within the scope of particular applications. In addition to these APs, a large amount of generic information, applicable to various kinds of products and applications, is used. This saves the AP developers from redundant effort. Each AP contains a number of important elements, including a scope for the AP, application reference model (ARM) which describes the information requirements and constraints in the terminology of that particular domain, application interpreted model (AIM) that is a representation of the ARM in terms of STEP constructs, ${ }^{12}$ and methods for testing conformance of an implementation of the standard (conformance testing, abbreviated as CT).

The effort to extend STEP to apparel product data has been undertaken by the Apparel Product Data Exchange Standard (APDES) project at NIST. This project is funded by the Defense Logistics Agency (DLA) which is interested in streamlining the process of contracting uniform design and manufacture through adoption of integration standards; and improving garment fit by replacing the traditional ready-to-wear sizing with a made-to-measure system.

A prototype AP (to be used as a straw man for an official ISO STEP AP and containing all the parts of an STEP AP except for the AIM) for ready-to-wear pattern making has been under development at NIST. The AP covers ready-to-wear pattern making, focusing on the "representation of two-dimensional (flat) patterns generated by the traditional ready-to-wear pattern making and grading method."13 A prototype AP for made-to-measure pattern making is also under development. The ultimate goal, of course, is to incorporate all information that describes an apparel product in terms of STEP.

Other work related to apparel product data is being done by AAMA/ARC. As mentioned earlier, ARC has published an apparel pattern data interchange standard approved by ANSI. This standard is based largely upon the Drawing Interchange file format (DXF) developed by AutoDesk ${ }^{\mathrm{TM}}$, Inc. for their AutoCAD® product ${ }^{14}$. In addition to continuing research, ARC is tasked with promoting

\footnotetext{
${ }^{11}$ Schenck. 1994.

12 Lee \& Moncarz, p. vi. 1994.

13 Lee \& Moncarz, p. iii. 1994.

14 ANSI/AAMA-001-1992 : "Standard for Pattern Data Interchange - Data Format."
} 
the move towards CIM standards within the apparel community and identifying technologies that will enable the U.S. apparel sector to become more competitive globally ${ }^{15}$. Current product data work includes developing implementation guidelines for the pattern data interchange standard, a standard for grade rule table exchange to support the pattern data exchange standard, and a plotter data exchange standard ${ }^{16}$.

The area of integration standards is one that seems to warrant special attention by those in the FTA industry, especially in the apparel sector, where losses to foreign competition are greatest (due to intensive labor requirements). A large portion of the apparel sector is made up of small and medium-sized companies who lack the resources to develop their own standards and protocols. Potential exists for increasing efficiency through integration and automation standards development (and implementation).

\section{TEST METHODS}

In order for an industry with hundreds of suppliers selling (what is supposed to be) the same product to hundreds (or even thousands) of buyers, standards are needed to insure that products of the same type are uniform (and to rate goods based on their quality). FTA is such an industry, where multitudes of cotton growers and wool farms sell tens of thousands of tons of raw fiber to the fabric manufacturers who, in turn, sell many bolts of colored fabric to the apparel manufacturers. It is absolutely essential that the apparel sewers, who produce the end product, have materials to work with that are of high and consistent quality.

At virtually every step in the transformation of raw fibers to finished apparel, inspections are made and tests are done. Specific physical (and sometimes chemical) properties of the fiber, or textile, or apparel are tested to insure that they meet the requirements of the manufacturer and its buyers. It is important that each company uses the same tests for the same property, so that the results can be interpreted consistently by those working with the manufacturer and their customers. To this end, standards organizations are formed and standard test methods and procedures created and published.

Most test methods consist of three main sections: purpose and scope, procedure, and evaluation method. The purpose and scope describe exactly what property is to be tested by the method and to what type or types of fibers or fabrics or yarns it pertains. The procedure section is at the heart of the test, and explicitly describes what steps to take in order to perform the test. The procedure details what supplies, chemicals, or special equipment to use and how to use them. Lastly, the evaluation section tells the tester what exactly to look for in rating the particular property being observed and very often refers to a control sample or a standard reference system, such as the American Association of Textile Chemists and Colorists (AATCC) Chromatic Transference Scale ${ }^{17}$.

Test methods apply to the fiber and textile segments of the industry, but in general not the apparel sector, as the physical properties that can be tested completely objectively have already been taken care of. Evaluation of finished apparel garments are done by means of quality standards and specifications, which are covered in a later section. Test methods relating to the fiber and textile sectors of the FTA industry are described in turn below.

15 Moncarz \& Lee 1. 1994.

16 AAMA. 1995.

17 AATCC Evaluation Procedure 3. "Chromatic Transference Scale." p. 351 of AATCC Technical Manual. 


\subsection{Fibers}

The fiber sector harvests raw natural fibers (or produces raw man-made filaments) and sells these fibers to the textile sector. The most basic properties of these fibers (and filaments) need to be known by both sectors. To this end, many tests are performed and their results recorded. The main properties of interest include length and length distribution, strength and elongation, maturity, and adhesion to other materials, such as steel or rubber. These properties are important because they directly relate to how the fibers will act during the spinning process.

The tests used for fibers and textiles are created and published by two main organizations. They are the American Society for Testing and Materials (ASTM), and ISO TC 38. With respect to leather goods, the American Leather Chemists Association (ALCA) publishes standards, as does ISO. Approximately 90 of ALCA's 140 or so test methods have been adopted by ASTM. These organizations play a key role in the development of standards. Since they are independent of any particular company, their standards are used throughout the sector. Having external standards also saves each grower or distributor from having to develop and adopt its own standards, which wouldn't be universal anyway.

Although some of the test methods apply to all types of fibers, most are specifically targeted at one type each. This is due to the intrinsic differences between man-made and natural fibers, and the further differences between cotton and wool (the natural fibers used most often). So, although the properties being tested are limited in number, the number of test methods are proliferated by the variety of fiber types.

\subsection{Textiles}

The business of the textile sector is to take raw fibers and filaments and convert them into fabrics which can then be sewn into garments. This process involves three main steps. In the first, the yarn manufacturer prepares the fibers or filaments (through carding, drawing, and roving), spins it into cones of yarn, and then winds the yarn onto spools. During the second stage, the slashing plant chemically treats the yarn, preparing it for the next step. The last, and most involved process is accomplished at the weaving plant. The yarn is woven (or knitted) into fabric first. After that, the fabric is prepared, dyed, and finished. Lastly, the fabric is cut for shipping to the garment sewing plants.

Throughout this process numerous checks are made. After every major step of the fabric manufacturing process, at least a visual inspection is done. Test methods applying to textiles are concerned with a wide range of features. These include strength, flammability, creasing, and dimensional change due to different environmental factors. The property that is most thoroughly tested is colorfastness. The importance of that particular behavior of a textile is shown in that almost half of ISO's 114 standards related to fabrics deal with colorfastness.

There are a few organizations that publish test methods for textiles. At the national level, AATCC and ASTM both make standards. ISO TC 38 publishes standards, including test methods, on the international level. As its name implies, AATCC is most concerned with chemical and biological properties of fabrics and colorfastness, though some physical properties are covered. ASTM tests are very physical in nature, dealing with aspects such as abrasion resistance, moisture, and mass. There are many more tests applying to textiles made by AATCC than by ASTM. ISO tests are dominated by tests for colorfastness, since many of the other textile-related standards are reference information and not test methods. 
A major part of the body of standards which affect the FTA industry are general quality standards or specifications. Unlike the integration standards and the test methods, quality standards are concrete expectations for a finished product of a certain type. The expectations conveyed through the document vary in content from flammability (such as a fireproof coat) to appearance, and vary in detail from a general durability specification to a military standard for a uniform specifying every design particular.

Quality standards are used for many different products in the FTA industry. In most cases, the standard applies to high-level concepts in a finished product, rather than minute details. For instance, there are not many quality standards applying to raw fibers, since examining most of the properties of those fibers require specific tests. In keeping with the organization philosophy, the quality standards have been sectioned on the basis of their area of application. The major areas of interest are general apparel, special and protective clothing, military specifications, and textile manufacturing machinery.

\subsection{General Apparel}

The majority of garments which are manufactured are sold to retailers who in turn sell them through stores. The success of the apparel sector, and to a large part the whole FTA industry, is determined by whether people, especially those in the United States, buy the clothes that the garment companies sew. It is of paramount importance that the garments put on the shelf be of consistently high quality, and it is in the interests of the manufacturers to minimize the number or seconds that cannot be sold for full price.

There are many standards used to ensure that garments sold to a customer satisfy minimum quality as defined by those standards. Most manufacturing and also retail companies have their own inspections, but national and international specifications do exist. These are written by ASTM on the national level. ASTM has about fifty performance specifications, each applying to a different type of apparel, such as knitted overcoat fabrics for men and women ${ }^{18}$, or swim wear ${ }^{19}$ fabrics. In addition, some smaller apparel manufacturers and retailers adopt the inspection criteria of large, established companies such as J.C. Penny, Inc., making such procedures de facto standards.

It is important to note that most of these specifications are standards of quality for the fabrics used to sew the garments. They insure that the clothing made will meet some basic standards of durability and, in some cases, fit. ASTM publishes several standards relating to fit, as opposed to the fifty or so fabric-related performance specifications mentioned earlier. The manufacturers and designers of clothing who use these standards still have the ability to make whatever they want, provided the material it is made from meets the specifications they have voluntarily adopted. In the end, it is beneficial for fabric manufacturers to use these quality standards so potential customers in the apparel sector will know that they are not buying shoddy materials.

\subsection{Special/Protective Clothing}

The area of special and protective clothing is one of the most sensitive to quality. This is for the obvious reason that the consequence of product failure is often injury to the wearer of the garment.

${ }^{18}$ D 3562 - 92 (ASTM) : "Performance Specification for Men's and Women's Sliver Knitted Overcoat and Jacket Fabrics."

${ }^{19}$ D 3994 - 94 (ASTM) : "Performance Specification for Men's, Women's, and Children's Woven Swimwear Fabrics." 
(This is much worse than merely inconveniencing or alienating a customer, the result of general apparel defects.) For this reason, quality standards must be more demanding and much less tolerant of deviations. As a result, the field of protective clothing has a relatively large number of fairly specific quality standards and specifications associated with it.

Standards relating to protective and other special clothing can be differentiated on the basis of what exactly the garment they relate to is intended to do (or in most cases prevent against). Special clothing is needed for use in a variety of hazardous environments; it may protect against electricity, chemicals, fire, or even cold. Because of the large number of fires and firefighters, fire-protective clothing is probably the most common protective clothing, though electrically insulated and chemical-protective clothing are very important in their respective industries.

Specifications for special clothing are published by ISO TC 94 on an international level. The National Fire Protection Association (NFPA) writes national requirements for protective clothing for fighting fires. There are many other standards that relate to protective clothing which are not quality standards, but rather test methods applied to the fabric from which these garments are made. These standards are published primarily by ASTM and ISO.

\subsection{Military Specifications and Standards}

The U.S. Armed Forces are probably the largest single customer for apparel made in the United States. The Department of Defense (DoD) spends hundreds of millions of dollars every year purchasing uniforms and other textile-based equipment. The consistent quality of garments purchased is highly valued by the military, more so than in the civilian market. In addition to the uniforms looking the same, they must meet strict requirements for durability and reliability, since many of them are ultimately intended for combat. It is also important that the clothing is functional and easy to wear under a wide variety of conditions. To insure the consistency, toughness, and utility of their uniforms, DoD publishes specifications generally referred to as the "MIL-" standards or specifications.

There are over $600 \mathrm{MIL}$-specifications that detail the requirements of specific apparel and textilerelated products and a dozen or so MIL standards that detail the requirements of a category of apparel and textile-related products. These specifications vary greatly in content. On one side of the spectrum, quality standards exist that cover all uses of certain fabrics or textiles in military equipment $^{20}$. At the other extreme, some ML-specifications are detailed requirements for the making of a certain garment ${ }^{21}$. There are also a substantial number of standards that involve textile products other than apparel. Examples of this would be fabric hoses and life preservers. Since these are still products of the FTA industry as a whole, they have been included within the scope of this survey.

Military specifications follow a specific format. Each has six sections-scope, applicable documents, requirements, quality assurance provisions, packaging, and notes. The scope section specifies exactly what the document applies to, for instance a polyester/cotton broadcloth durable press shirt. 22 The next section lists other documents that the manufacturer must adhere to in making the garment. These include federal and other military specifications and standards, as well as test methods published by private organizations such as AATCC and the American Iron and Steel Institute (AISI)-for steel rings, zippers, and fasteners. The third section details expectations, while the fourth section explains how those requirements are to be verified. The

\footnotetext{
20 MIL-C-429A : "Cloth, Twill, Nylon."

21 MIL-C-1509H : "Coat, Food Handler's (Steward)."

22 MIL-44041C(GL) : "Shirt, Man's, Short Sleeve, Polyester Cotton, Army Green 415, Durable Press."
} 
packaging section is self-explanatory. The last part of every MIL-specification contains information of a general or explanatory nature that may be helpful, but is not mandatory.

The current system of military specifications is designed to insure total uniformity. Every detail of the sewing process is dictated. There are typically a dozen or more other documents referenced in each $\mathrm{MLL}$-specification. The reference to each consists only of the name and number of the standard. No indication is given to the manufacturer of where to find the information that pertains directly to the making of the garment. Unless the scope of the item referred to is very narrow, this can make it difficult for the contractor to comply. Companies are left to search a possibly very large document from cover to cover to find what might be a very small section applicable to their product.

At the present time, proposals are being made to use commercial specifications because they are simpler. The format for the new series is known as a commercial item description (CID). The main difference is that the new format will specify what is desired, and allow the contractor to make it in the most efficient method availible. Previously, the MIL-documents gave exact instructions for making the item, which placed sometimes unnecessary demands on the companies contracted to do the job. In addition, some of the specifications will be given in terms of performance, rather than requiring a certain material, giving the maker leeway in choosing the most desirable way to meet the requirements. This will make the process of procuring uniforms faster and more efficient.

\subsection{Textile Machinery}

The process of making textiles from fibers and filaments is almost completely done by machine. Setting up and loading the equipment is still often done manually, but the actual spinning, weaving, etc. is done automatically. Therefore, the sector depends on these devices consistently working in the proper manner. Standards are used to insure the safety and reliability of textile machinery.

The primary publishers of specifications for textile machinery are ISO TC 72 and ASTM. Most of these documents apply to key pieces of the machines, such as the rings and travelers on ring spinning machines ${ }^{23}$, or the cones for yarn winding 24 . There are also a good number of standards which give definitions and terminology relating to different types of textile equipment. These will be discussed in the next section.

\section{STANDARD REFERENCE INFORMATION}

Standard reference information is necessary in any field where uniformity and consistency is important. This information makes repeatability possible by providing accepted standards that can be used for comparison purposes and computation purposes. For example, AATCC has a standard table for gray-scale color change ${ }^{25}$. This table is intended for use with the test methods they developed. Use of that table insures that the evaluation given to the textile will not depend on the tester, but rather be objective (with respect to the AATCC standard). The test results will also be reproducible.

\footnotetext{
23 ISO 96-1:1992 : "Textile Machinery and Accessories - Rings and Travellers for Ring Spinning and Ring Doubling Frames - Part 1: T-rings and Their Appropriate Travellers."

24 ISO 111:1978 : "Textile Machinery and Accessories - Cones for Yarn Winding (Cross Wound) - Half Angle of the Cone 4 Degress 20'."

25 AATCC Evaluation Procedure 1 : "Gray Scale for Color Change." p. 348 of AATCC Technical Manual.
} 
Standard reference information can be divided into three categories: standard reference data (SRD), standard reference materials (SRM), and terminology. These are described below.

\subsection{SRD}

Standard reference data (SRD) refers to a collection of scientific or technical measurements, values, or facts that can be represented quantitatively. SRD is accepted as correct within a particular domain of expertise to be used as the basis of further calculations or decisions. A very simple example from the field of engineering is the assignment of the value for the constant $\pi$. $\Pi$ is the ratio of a circle's circumference to its diameter, and its value can only be estimated to a specified level of precision. For the purpose of taking a test, students may be told to use the value of 3.14 for $\pi$. Therefore, they should all get the same answer, and their answers should conform with the professor's solutions. Although simple and far removed from the FTA industry, this analogy illustrates both the nature of SRD and its significance.

It is easy to see the importance of these accepted values when the opposite scenario is considered. If there was no accepted value for $\pi$, each student would make an independent best guess, or use whatever approximation the student felt appropriate. Some might use 3.14, others might extend it to five or six places, while a handful might just truncate the fraction and go with 3 . More ambitious students might use string and ruler to measure the constant directly from a circular object. (Others might forget entirely and just guess 7.) Depending on what is being done with the number, the end results could be drastically different (and in some cases drastically wrong).

In the apparel industry, an important set of standard reference data are the different dimensions that make up size. To achieve a good fit, the apparel manufacturer needs accurate measurements of the human body. This is called anthropometric data. The first standard set of body dimensions was compiled by the National Bureau of Standards (NBS, now NIST) in the 1950's. In 1983, the Department of Commerce withdrew these voluntary standards. ASTM took over responsibility. The D-13.55 Body Measurement for Apparel Sizing sub-committee of ASTM has published standard tables of measurements for ladies ${ }^{26}$, infants ${ }^{27}$, and women over fifty-five ${ }^{28}$. Sizing standards for children, men, and large women are in different stages of committee review. With the exception of the sizing for women over 55, all of these standard tables are based on the original anthropometric survey conducted by NBS. D-13.55 is currently trying to rally industry support to update the anthropometric survey to reflect the changing population of the country. Internationally, ISO TC 133 has an international standard of anthropometric data 29 and sizing.

From surveys of body measurements, standards for actual sizing of garments are derived. NBS had developed close to twenty voluntary apparel sizing standards which it published in the late 1960s. These covered all the most common types of apparel, from shirts to gloves. Although girls and women were part of the anthropometric survey, there were no voluntary standards relating specifically to women's clothing. The NBS-sizing standards were withdrawn in 1983. On an international level, ISO TC 133 publishes ten standards relating to clothing size for both sexes. A bibliography dealing with apparel sizing was published by NIST in $1994^{30}$.

26 ASTM D 5585 - 93. "Standard Table of Body Measurements for Adult Female Misses Figure Type Size 2-20."

27 ASTM D 4910 - 89. "Standard Table of Body Measurements for Infants, Ages 0 to 18 Months."

28 ASTM D 5586 - 94. "Standard Tables of Body Measurements for Women Aged 55 and Older (All Figure Types)."

29 ISO 8559:1989. "Garment Construction and Anthropometric Surveys — Body Dimensions."

30 Lee $1,1994$. 
Although the NBS anthropometric data and sizing recommendations were valuable, some larger manufacturers have done work to improve the fit of their garments for their customer population. Of the companies in the U.S., Sears, Roebuck and Company, Inc. has the distinction of doing the most body size and clothing fit research. ${ }^{31}$ With the knowledge they have gained, they publish pages of details on sizing and fitting of garments. Although Sears has placed special emphasis on this in the past, reducing returns and increasing customer satisfaction through improving the way apparel fits remains a goal of all clothing manufacturers.

\subsection{SRM}

Standard reference materials are physical artifacts that are used for direct comparison with the sample being evaluated. The reference material is accepted as a standard for the property it exemplifies. SRMs are often used when dealing with qualitative aspects of an item, such as color or texture. ("Qualitative aspects," as used here, refers to those properties that are generally not measured by the industry directly due to technology limitations. For example, as technology advances, measurements of texture may be more scientifically conducted than by a comparison with known textures, as it is generally done in the textile industry today.) In order to have some degree of consistency and control over properties, the properties must be converted to a quantitative base. This is done by selecting an arbitrary point of reference which the property of a particular physical artifact exudes. Then samples may be measured relative to the "standard," consequently providing an objective measurement of the "qualitative" property.

Many, if not most, of the pertinent properties of fibers, textiles, and apparel are qualitative. However, many of these properties can be quantified through a certain method of testing. A few can not. As mentioned earlier, a large percentage of the test methods relating to the fiber and textile sectors relate to colorfastness. AATCC has developed scales for evaluating color change (mentioned before), as well as transference ${ }^{32}$ and staining ${ }^{33}$ reference standards. These SRMs are directly compared with the sample that has been through the test procedure (and also a control sample in tests relating to color change).

Another type of SRM which warrants mention is the model form. Model forms are actual molds of the human body used to check sizing for apparel. NBS made standard model forms for girls', boys', and toddlers' apparel of different sizes. These were developed in conjunction with the anthropometric survey discussed above in Section 6.1. Although these may be used for reference, apparel manufacturers have their own model forms for all types of people and sizes.

\subsection{Terminology}

The largest number of reference standards developed for the FTA industry relate to vocabulary and definitions. Standard terminology is very important because it facilitates communication. Since some words have multiple meanings, and there are many ways to describe or designate a certain object, discussion can often become obfuscated. Having precise definitions for key items and ideas in a field has always been the responsibility of that area's standards organizations. The FTA industry is no exception.

The task of publishing definitions and vocabulary on an international level has been undertaken by ISO. There are approximately forty-five ISO standards which define terminology for everything

31 Hudson, pp. 121-122. 1983.

32 AATCC Evaluation Procedure 3. "Chromatic Transference Scale." p. 351 of AATCC Technical Manual.

33 AATCC Evaluation Procedure 2. "Gray Scale for Staining." p. 350 of AATCC Technical Manual. 
from stitches ${ }^{34}$ to fibers ${ }^{35}$. Some of the standards which fall into this category deal with words, while a slightly smaller number define some physical aspect of a piece of equipment, such as which side is left and which is right ${ }^{36}$. ISO's terminology standards are most heavily concentrated in the area of textile machinery, where there are many different types of machines, each with a plethora of parts that may need definitions to refer to them.

ASTM has written roughly fifteen standards defining terminology for the FTA industry. Over half of these standards deal with textiles (yarns and fabrics) and textile properties, while a smaller number deal with the textile manufacturing and apparel sewing processes. A few of the documents apply to labeling of apparel. There is a terminology specifically for wool ${ }^{37}$, but not for the other fibers. This may be because wool requires a lot of processing before it can be spun into yarn. Dealing with plant fibers such as cotton and flax, is simpler. One standard of special interest to the apparel sector defines terminology for apparel sizing 38 . Overall, these documents seem to cover a good portion of the industry.

\section{SUMMARY}

The primary purpose of this survey was to identify the standards that apply to the U.S. FTA industry. To compete effectively in the global marketplace, the FTA industry must operate as efficiently as possible. By developing and adopting new standards where they are needed, and improving existing standards where possible, many benefits in terms of reduced wait time and elimination of unnecessary effort can be realized by the industry as a whole.

As can be seen by a perusal of the appendix, the number of standards related to the FTA industry is voluminous. The intent of this paper was to bring together in one document a listing of the standards and standards' organizations associated with the FTA industry. That compilation represents a first step to determine where to concentrate resources on further standards' development.

Industry feedback is necessary to draw conclusions concerning the prioritization of future standards' efforts. For example, in what parts of the FTA manufacturing process are the current standards effective? What is it about those standards and the way they are implemented that makes them effective? Where does there seem to be a lack of unity in standards-where different standards are used by different people for the same purpose? The answers to these and other questions can provide insight into where standards are helping and where they are holding back the FTA industry, and how improvement of the standards can make the FTA industry more competitive.

34 ISO 4915:1991. "Textiles - Stitch Types - Classification and Terminology." Bilingual Edition.

35 ISO 8159:1987. "Textiles - Morphology of Fibers and Yarns - Vocabulary." Bilingual Edition.

36 ISO 92:1976. "Textile Machinery and Accessories - Spinning Machinery - Definition of Side (Left or Right)."

37 ASTM D 4845 - 89. "Terminology Relating to Wool."

38 ASTM D 5219-94. "Terminology Relating to Body Dimensions for Apparel Sizing." 



\section{APPENDICES}

\section{A REFERENCES}

Accredited Standards Committee X12. The ASC X12 Plan for Technical Migration to and Administrative Alignment with UN/EDIFACT (Amended). May, 1995.

American Apparel Manufacturers Association. Minutes for November 1995 Meeting. Arlington, VA.

American Association of Textile Chemists and Colorists. AATCC Technical Manual. Research Triangle Park, NC : 1994.

American Leather Chemists' Association. Journal of the American Leather Chemists' Association. Vol. 89, June 1994. Cincinnati, OH.

American Leather Chemists' Association. Methods of Sampling and Analysis. Cincinnati, $\mathrm{OH}$ : 1994.

American Society for Testing and Materials. 1994 Annual Book of ASTM Standards. Section 7, Textiles. Vol. 07.01 and 07.02. Philadelphia, PA : 1994.

Arnoff, Robert and Hsing, Karen. An Analysis of ANSI ASC X12 and UN/EDIFACT Electronic Data Interchange (EDI) Standards. NISTIR 5631, National Institute of Standards and Technology. Gaithersburg, MD : $1995^{39}$.

Fowler, Julian. STEP Development Methods. Australian STEP Conference : Sydney, 17-18 March 1995.

Gaffin, Adam. "EDI Standards Migration Effort Delayed Till 1999; Group to Help X12 Users Ease Into EDIFACT." Network World. Oct. 10, 1994, v11, n41.

Hudson, Peyton B. Guide to Apparel Manufacturing. MEDIApparel, Inc. Greensboro, NC : 1983.

Information Handling Services, Inc. Government/Military Standards and Specifications Service Locator Index. Englewood, CO : 1994.

Information Handling Services, Inc. VSMF Product Subject Index. Englewood, CO : 1994.

International Organization for Standardization. ISO Catalogue 1994. Geneva, Switzerland : 1994.

Lee, Y. Tina. A Bibliography on Apparel Sizing and Related Issues. NISTIR 5365, National Institute of Standards and Technology. Gaithersburg, MD : 1994.

Lee, Y. Tina. Body Dimensions for Apparel. NISTIR 5411, National Institute of Standards and Technology. Gaithersburg, MD : 1994.

${ }^{39}$ Reports from the National Institute of Standards and Technology are availible from the National Technical Information Service, Springfield, VA 22161. 
Lee, Y. Tina and Moncarz, Howard T. A Prototype Application Protocol for Ready-to-Wear Pattern Making. NISTIR 5115, National Institute of Standard and Technology. Gaithersburg. MD : 1993.

Link, Paul. Textile Encyclopedia. Taleres Graficos Jorman. Buenos Aires : 1954.

McCarthy, Shawn P. "Why Agencies Are So Slow to Embrace EDI," Government Computer News. March 20, 1995, v14, n6. Cahners Publishing Associates.

Markovitz, Paul. Electronic Data Interchange in Message Handling Systems. NISTIR 4608, National Institute of Standards and Technology. Gaithersburg, MD : 1991.

Moncarz, Howard T. Information Technology Vision for the U.S. Fiber/Textile/Apparel Industry. NISTIR 4986, National Institute of Standards and Technology. Gaithersburg, MD : 1992.

Moncarz, Howard T. and Lee, Y. Tina. "CALS Technologies Applied to the Fiber/Textile/Apparel Industry." CALS/Enterprise Integration Journal. Summer 1994.

Moncarz, Howard T. and Lee, Y. Tina. Report on Scoping the Apparel Manufacturing Enterprise. NISTIR 5106, National Institute of Standards and Technology. Gaithersburg, MD : 1993.

National Fire Protection Association. NFPA Catalog. The. Quincy, MA : 1995.

Riverside Publishing Company, The. Webster's II : New Riverside University Dictionary. Houghton Mifflin Company : Boston, MA : 1984

Shaw, Jack and Olson, Paula. "An EDI Review (1994 PC Expo Show)." The Computer Conference Analysis Newsletter. July 6, 1994, n345. Guidelines.

Schenck, Douglas and Wilson, Peter, Information Modeling: The EXPRESS Way, Oxford University Press, 1994.

Society of Automotive Engineers, Inc. SAE AMS Index. Warrendale, PA : 1994.

Textile-Apparel Linkage Council/Sundries and Findings Linkage Council. TALC/SAFLINC Conventions and Implementation Guidelines. American Apparel Manufacturer's Association. Arlington, VA : 1994.

Textile Clothing Technology Corp. Process Steps for Men's Cotton Slacks. EMS95-108, Version 1.1.1994. For detailed information contact Dr. J.F. Macfarlane, Lawrence Berkeley National Laboratory.

U.S. Allied-Textile Industry Standards Coalition. International Standards Issues and the U.S. Allied-Textile Industry. American Textile Manufacturer's Insatiate. Washington, DC : 1995.

U.S. Department of Commerce/National Bureau of Standards. List of Product Standards, Commercial Standards, and Simplified Practice Recommendations. Washington, DC : 1968.

Uniform Code Council, Inc. What is Electronic Data Interchange: UCS VICS EDI Wins. Dayton, $\mathrm{OH}: 1994$. 


\section{B FTA STANDARDS ORGANIZATIONS}

The following is a list of organizations publishing and/or developing standards and specifications related to the FTA industry. This listing of organizations is intended to save time by bringing them together in one place. In addition to the contact information, a short description and sometimes notes are included beside each listing.

American Apparel Manufacturers Association (AAMA) 2500 Wilson Blvd., Suite 301

Arlington, VA 2201

(703) 524-1864

FAX: (703) 522-6741

American Association of Textile Chemists and Colorists (AATCC)

One Davis Drive

P.O. Box 12215

Research Triangle Park, North Carolina 27709

(919) 549-8141

FAX: (919) 549-8933

American Leather Chemists Association (ALCA)

Tanners Bldg.

University of Cincinnati-Loc. 14

Cincinnati, Ohio 45221

(513) 556-1197

FAX: (513) 556-2377

American National Standards Institute (ANSI)

11 W. 42nd Street, 13th Floor

New York, New York 10036

(212) $642-4900$

FAX: (212) 398-0023

American Society for Testing and Materials (ASTM)

1916 Race Street

Philadelphia, Pennsylvania 19103-1187

(215) 299-5585

FAX: (215) 977-9679

American Textile Manufacturers Institute. Inc. (ATMT)

1801 K Street, NW, Suite 900

Washington, D.C. 20006

(202) 862-0500

FAX: (202) 862-0570
Sanctioned by ANSI ${ }^{40}$ to create standards for the apparel sector of the FTA Industry. Responsible for TALC/SAFLINC voluntary integration standards.

Responsible for test methods and procedures relating to physical and chemical properties of textiles. Sanctioned by ANSI.

Publishes test methods for evaluating raw leather and leather products. Most standards adopted by ASTM.

Sanctions standards from industry organizations in all fields for use on a national level.

Publishes standards covering many different materials. D-13 Committee responsible for textiles. Uses ALCA standards for leather.

Responsible for FASLINC standards.

40 "Sanctioned by ANSI" means that many or most of the standards they publish are approved and adopted by ANSI as U.S. national standards. 
International Organization for Standardization (ISO)

1 , rue de Varembé

Case postale 56

$\mathrm{CH}-1211$ Genéve 20

Switzerland

$+41227490111$

FAX: + 41227333430

National Fire Protection Association

One Batterymarch Park

P.O. Box 9101

Quincy, Massachusetts 02269-9101

(617) $770-3000$

FAX: (617) 770-0700

SAE International (SAE)

400 Commonwealth Drive

Warrendale, Pennsylvania 15096-0001

(412) 776-4841

FAX: (412) 776-4026

Uniform Code Council

8163 Old Yankee Road, Suite J

Dayton, Ohio 45458

(513) $435-3870$
Standards relating to almost all fields. Members from 100

countries. 182 technical committees (TCs), 630 subcommittees. ${ }^{41}$ TCs of interest include 38 - Textiles, 72Textile Machinery, 94 - Protective Clothing, and 133 - Sizing Systems.

Responsible for standards and codes relating to fire safety. These include specifications for protective clothing (primarily for fire fighting).

Publishes specifications for highperformance textiles such as aramidfiber.

Responsible for UCS and VICS retail EDI standards.

The following organizations are not directly involved in writing standards, but serve other important capacities related to FTA standards.

American Textile Partnership (AMTEX)

Laboratory Program Office

Pacific Northwest Laboratory

P.O. Box 999

Richland, WA 99352

(509) $375-2306$

Industry Program Office

P.O. Box 4670

Wilmington, DE 19807

(302) 999-6733

FAX: (302) 999-6736

National Institute of Standards and Technology (NIST)

Manufacturing Systems Integration Division

Room A127, Bldg. 220

Gaithersburg, Maryland 20899

(301) 975-3508

Collaboration of FTA industry and

DOE. Develops technologies to address industry needs. Helps industry to optimize product quality and market responsiveness while minimizing costs and environmental impacts.

FAX: (301) 258-9749

AMTEX projects are coordinated through the Laboratory Program office (of DOE) and the Industry Program Office.

Current efforts include the Apparel Product Data Exchange Standard (APDES) project.

${ }^{41}$ ISO Catalogue 1994. p. 7. 


\section{FTA STANDARDS LISTINGS}

The following is a listing of FTA standards obtained from the organizations listed in Appendix B. The listings were obtained when possible from the organizations that issue the standards. The listings have been reformatted so that they will be consistent across the standards organizations. For the most recent information or to purchase any of these standards, contact the appropriate organization directly. Information for contacting any of these organizations can be found in Appendix B: FTA Standards Organizations.

\section{C.1 AAMA Standards}

The American Apparel Manufacturers Association is in the process of creating and publishing a number of standards which are important to the integration of apparel manufacturing. The standards which have been generated thus far are the following:

ANSI/AAMA-001-1992

ANSI/AAMA-292-1993
Standard for Numerically Controlled Cutting Machines.

Standard for Pattern Data Interchange - Data Format. 


\section{C.2 AATCC Test Methods and Procedures ${ }^{42}$}

The standards listed are organized first according to the following categories:

\section{BIOLOGICAL PROPERTIES \\ COLORFASTNESS \\ DYEING PROPERTIES \\ EVALUATION PROCEDURES \\ IDENTIFICATION AND ANALYSIS \\ PHYSICAL PROPERTIES}

Within each category standards are listed in numerical order, according to their identification number in the left column. All standards are test methods unless otherwise noted.

\section{BIOLOGICAL PROPERTIES}

24-1993

28-1994

30-1993

100-1993

103-1994

147-1993

174-1993

2-1989

3-1989

6-1994

8-1989

8-1989

9-1989

11-1989

15-1994

16-1993

16-1993

23-1994

61-1994

101-1994

104-1994

106-1991

107-1991

109-1992

116-1994

117-1994
Insects, Resistance of Textiles to, p. 75.

Insect Pest Deterrents on Textiles, p. 83.

Antifungal Activity, Assessment of Textile Materials: Mildew and Rot Resistance of Textiles, p. 85.

Antibacterial Finishes of Textile Materials, Assessment of, p. 148. Bacterial Alpha-Amylase Enzymes Used in Desizing, Assay of, p. 154.

Antibacterial Activity of Fabrics, Assessment of Textile Materials: Parallel Streak Method, p. 261.

Antimicrobial Activity Assessment of Carpets, p. 328.

\section{COLORFASTNESS}

Colorfastness to Fulling, p. 174.

Colorfastness to Bleaching with Chlorine, p. 19.

Colorfastness to Acids and Alkalis, p. 21.

Colorfastness to Crocking: AATCC Crockmeter Method, p. 23.

Colorfastness to Crocking: Rotary Vertical Crockmeter Method, p.23.

Colorfastness to Stoving, p. 26.

Colorfastness to Carbonizing, p. 28.

Colorfastness to Perspiration, p. 30.

Colorfastness to Light, p. 33.

Colorfastness to Light, p. 241.

Colorfastness to Burnt Gas Fumes, p. 72.

Colorfastness to Laundering, Home and Commercial; Accelerated, p. 94.

Colorfastness to Bleaching with Hydrogen Peroxide, p. 150.

Colorfastness to Water Spotting, p. 1565.

Colorfastness to Water: Sea, p. 157.

Colorfastness to Water; , p. 159.

Colorfastness to Ozone in the Atmosphere under Low Humidities, p. 161.

Colorfastness to Degumming, p. 192.

Colorfastness to Heat: Dry (Excluding Pressing), p. 194.

42 AATCC Technical Manual. pp. 5-14. 1995. All page numbers in this section refer to this document. 
119-1994

120-1994

125-1991

126-1991

129-1990

131-1990

132-1993

133-1994

139-1989

145-1985

153-1985

$157-1990$

$162-1991$

163-1992

164-1992

165-1993

$172-1990$

173-1992

177-1993

140-1992

141-1994

146-1994

154-1991

155-1991

156-1991

159-1994

161-1992

166-1993

167-1993

170-1989

176-1993

Evaluation Procedure 1 Evaluation Procedure 2 Evaluation Procedure 3 Evaluation Procedure 4 Evaluation Procedure 5
Color Change Due to Flat Abrasion (Frosting) Screen Wire Method, p. 202.

Color Change Due to Flat Abrasion (Frosting) Emery Method, p. 202.

Colorfastness to Water and Light: Alternate Exposure, p. 214. Colorfastness to Water (High Humidity) and Light: Alternate Exposure, p. 215.

Colorfastness to Ozone in the Atmosphere under High Humidities, p. 219.

Colorfastness to Pleating; Steam Pleating, p. 30.

Colorfastness to Dry-cleaning, p. 225.

Colorfastness to Heat; Hot Pressing, p. 228.

Colorfastness to Light; Detection of Photochromism, p. 241.

Color Measurement of the Blue Wool Lightfastness Standards:

Instrumental, p. 256.

Color Measurement of Textiles: Instrumental, p. 272.

Colorfastness to Solvent Spotting: Perchloroethelyene, p. 284.

Colorfastness to Water: Chlorinated Pool, p. 297.

Colorfastness : Dye Transfer in Storage; Fabric-to-Fabric, p. 299.

Colorfastness to Oxides of Nitrogen the Atmosphere Under High

Humidities, p. 301.

Colorfastness to Crocking: Carpets - AATCC Crockmeter

Method, p. 303.

Colorfastness to Non-Chlorine Bleach in Home Laundering, p.

321.

CMC: Calculation of Small Color Differences for Acceptability, $p$. 324.

Colorfastness to Light at Elevated Temperature and Humidity;

Water Cooled Xenon Lamp Apparatus, p. 336.

\section{DYEING PROPERTIES}

Disperse and Vat Dye Migration: Evaluation of, p. 243.

Compatibility of Basic Dyes for Acrylic Fibers, p. 245.

Dispersibility of Disperse Dyes: Filter Test, p. 258.

Thermal Fixation Properties of Disperse Dyes, p. 278.

Transfer of Disperse Dyes on Polyester, p. 280.

Transfer of Basic Dyes of Acrylics, p. 282.

Transfer of Acid and Premetallized Acid Dyes on Nylon, p. 288.

Chelating Agents: Disperse Dye Shade Change Caused by Metals;

Control of, p. 262.

Dispersion Stability of Disperse Dyes at High Temperature, p. 305.

Foaming Propensity of Disperse Dyes, p. 307.

Dusting Propensity of Powder Dyes: Evaluation of, p. 317.

Speckiness of Liquid Colorant Dispersions: Evaluation of, p. 335.

\section{EVALUATION PROCEDURES}

Gray Scale for Color Change, p. 348.

Gray Scale for Staining, p. 350.

Chromatic Transference Scale, p. 351.

Standards Depth Scales for Depth Determination, p. 352.

Fabric Hand: Subjective Evaluation of, p. 353. 


\section{IDENTIFICATION AND ANALYSIS}

20-1990

20A-1989

78-1989

81-1989

82-1989

89-1989

94-1992

97-1989

98-1989

102-1992

110-1989

$112-1993$

144-1992

149-1992

168-1992

173-1992

$17-1989$

22-1989

26-1989

27-1989

35-1989

42-1989

43-1989

62-1989

66-1990

$70-1989$

76-1989

79-1992

84-1989

86-1989

88B-1992

88B-1992

92-1989

93-1989

96-1993

99-1993
Fiber Analysis: Qualitative, p. 47.

Fiber Analysis: Quantitative, p.63.

Ash Content of Bleach Celluloid Textiles, p. 105.

$\mathrm{pH}$ of the Water-Extract from Bleached Textiles, p. 107.

Fluidity of Dispersion of Cellulose from Bleached Cotton Cloth,

p. 108.

Mercerization in Cotton, p. 123.

Finishes in Textiles; Identification, p. 130.

Extractable Content of Greige and/or Prepared Textiles, p. 141.

Alkali in Bleach Baths Containing Hydrogen Peroxide, p. 142.

Hydrogen Peroxide by Potassium Permanganate Titration:

Determination of, p. 1552.

Whiteness of Textiles, p. 163.

Formaldehyde Release from Fabric, Determination of; Sealed Jar

Method, p. 183.

Alkali in We Processed Textiles: Total, p. 254.

Chelating Agents: Chelation Value of Aminopolycarboxylic Acids and Their Salts, Calcium Oxalate Method, p. 265.

Chelating Agents: Active Ingredient Content of Poly amino polycarboxylic Acids and Their Salts; Copper PAN Method, p.

311.

CMC: Calculation of Small Color Differences for Acceptability, $p$. 326.

\section{PHYSICAL PROPERTIES}

Wetting Agents, Evaluation of, p. 45.

Water Repellency: Spray Test, p. 70.

Aging of Sulfur-Dyed Textiles: Accelerated, p. 80.

Wetting Agents, Evaluation of Rewetting Agents, p. 82.

Water Resistance: Rain Test, p. 89.

Water Resistance: Impact Penetration Test, p. 91.

Wetting Agents for Mercerization, p. 93.

Oils, Wool: Oxidation in Storage, p. 98.

Wrinkle Recovery of Fabrics: Recovery Angle Method, p. 99.

Water Repellency: Tumble Jar Dynamic Absorption Test, p. 101.

Electrical Resistivity of Fabrics, p. 103.

Absorbency of Bleached Textiles, p. 106.

Electrical Resistivity of Yarns, p. 111.

Dry-cleaning: Durability of Applies Designs and Finishes, p. 113.

Appearance of Seams in Durable Press Items after Repeated Home Laundering, p. 115.

Smoothness of Seams in Fabrics after Repeated Home

Laundering, p. 218.

Chlorine, Retained, Tensile Loss: Single Sample Method, p. 125.

Abrasion Resistance of Fabric: Accelerator Method, p. 127.

Dimensional Changes in Commercial Laundering of Woven and Knitted Fabrics Except Wool, p. 137.

Dimensional Changes of Woven Knitted Wool Textiles:

Relaxation, Consolidation and Felting, p. 144. 
111A-1990

111B-1990

111C-1990

111D-1990

115-1989

118-1992

121-1989

122-989

123-1989

124-1992

127-1989

128-1989

128-1989

130-1990

134-1991

135-1992

136-1989

137-1989

138-1992

142-1989

143-1992

150-1992

151-1990

152-1990

158-1990

169-1990

171-1989

175-1993

178-1992

1114-1989

1600-1992

1 88C-1992
Weather Resistance: Sunshine Arc Lamp Exposure with Wetting, p. 175.

Weather Resistance: Exposure to Natural Light and Weather, p. 171.

Weather Resistance: Sunshine Arc Lamp Exposure without Wetting, p. 175.

Weather Resistance: Exposure to Natural Light and Weather through Glass, p. 165.

Electrostatic Clinging of Fabrics: Fabric to Metal Test, p. 188.

Oil Repellency: Hydrocarbon Resistance Test, p. 198.

Carpet Soiling: Visual Rating Method, p. 206.

Carpet Soiling: Service Coiling Method, p. 206.

Carpet Soiling: Accelerated Soiling Method, p. 208.

Appearance of Fabrics after Repeated Home Laundering, p. 210.

Water Resistance: Hydrostatic Pressure Test, p. 216.

Wrinkle Recover of Fabrics: Appearance Method, p. 217.

Wrinkle Recovery of Fabrics: Appearance Method, p. 217.

Soil Release: Oily Stain Release Method, p. 221.

Electrostatic Propensity of Carpets, p. 230.

Dimensional Changes in Automatic Home Laundering of Woven on Kit Fabrics, p. 233.

Bond Strength of Bonded and Laminated Fabrics, p. 236.

Rug Back Staining of Vinyl Tile, p. 239.

Shampooing: Washing of Textile Floor Covering, p. 240.

Appearance of Flocked Fabric after Repeated Home Laundering and/or Coin-Op Dry-Cleaning, p. 247.

Appearance of Apparel and Other Textile End Products After

Repeated Home Laundering; Text, p. 249.

Dimensional Changes in Automatic Home Laundering of

Garments.

Soil Redeposition, Resistance to: Launder-Ometer Method, p. 270.

Soil Redeposition, Resistance to: Terg O-Tomoeter Method, p. 272.

Dimensional Changes on Dry-cleaning in Perchloroethylene:

Machine Method, p. 287.

Weather Resistance of Textiles: Xenon Lamp Exposure, p. 165.

Carpets: Cleaning of; Hot Water (Steam) Extracting Method, p. 321.

Satin Resistance: Pile Floor Coverings, p 334.

Barre: Visual Assessment and Grading, p. 345.

Chlorine, Retained, Tensile Loss: Multiple Sample Method, p.

186.

Dimensional Restoration of Knitted and Woven Fabrics after

Laundering, p. 292.

Retention of Creases in Fabrics after Repeated Home Laundering, p. 119. 


\section{C.3 ALCA Standards 43}

This standards listing contains the names and numbers of all ALCA's test methods and definitions. Most of them related to leather in general, and a few specifically apply to leather for footwear purposes. Some of these standards have been adopted and re-published by ASTM. The names of ALCA/ASTM standards are followed by their ASTM document number (in parentheses).

$\mathrm{A} 1$

A5

A6

$\mathrm{A} 10$

A11

$\mathrm{A} 12$

A13

A20

A21

A22

A25

A30

A31

A 40

A50

A60

B1

B2

B3

B4

B5

B8

B9

B10

B11

B12

B15

B16

B20

B30

C1

C5

C10

C11

D1

D5

D10

Analysis of Vegetable Tanning Materials - General (ASTM D4899)

Extraction of Raw and Spent Materials

Moisture in Raw and Spent Materials

Preparation of Solution of Liquid Extracts (ASTM D4901)

Preparation of Solution of Solid, Pasty and Powdered Extracts

(ASTM 4905)

Cooling of Analytical Solutions (ASTM D4904)

Evaporation and Drying of Analytical Solutions (ASTM 4902)

Total Solids and Water (ASTM D4903)

Soluble Solids and Insolubles

Nontannins and Tannin

Analysis of Tannery Liquors

Sugar in Tanning Materials

Method for Copper and Iron in Tanning Materials

Color Tests with Sheepskin Skiver

Lignosulfonates (Sulfite Cellulose) (ASTM D4900)

Official Certification

Analysis of Vegetable-Tanned Leathers - General

Preparation of Sample for Analysis (ASTM D2813)

Moisture (ASTM D3790)

Hexane Extract of Leather (ASTM D2876)

Nitrogen Content (Kjeldahl) and Hide Substance (ASTM D2868)

Water-Soluble Matter of Vegetable-Tanned Leather (ASTM D2876)

Soluble Non Tannin and Uncombined Tannin

Glucose

Insoluble Ash of Vegetable-Tanned Leather (ASTM D2875)

Combined Tannin and Degree of Tannage

Total Ash in Leather (ASTM D2617)

Magnesium as Epsom Salts

$\mathrm{pH}$ of Water (ASTM 2810)

Official Certification

Determination of Chromium in Chrome Tanning Liquors (ASTM D3898)

Determination of Acidity of Chrome Tanning Liquors (ASTM D3813)

Calculation Basicity of Chrome Tanning Liquors (ASTM D3897)

Determination of $\mathrm{pH}$ of Chrome Tanning Liquors (ASTM D2815)

Preparation of Composite Sample for Chemical Tests (ASTM D2813)

Mineral Leathers - General

Chromic Oxide in Leather (Perchloric Acid Oxidation) (ASTM 2807)

43 Methods of Sampling and Analysis. 1994. 
D20

D21

D30

D35

E1

E2

E3

E4

E5

E10

E11

E12

E13

E14

E15

E16

E17

E30

E32

E40

E41

$\mathrm{E} 42$

E43

E44

E45

E46

E50

E52

E53

E54

E55

E56

E57

E58

E59

E60

E61

E62

E63

E64

F1

F3

F5

F10

Sulfates (Total, Neutral and Combined Acid) (ASTM D1655)

Total Chlorides (D4563)

Sulfate Basicity (ASTM D4654)

Acidity (pH) (ASTM D2810)

Conditioning Leather and Leather Products for Testing (ASTM

D1610)

Measuring Area of Leather Test Specimens (ASTM D2346)

Measuring Thickness of Leather Units (ASTM D1814)

Measuring Thickness of Leather Test Specimens (ASTM D1813)

Width of Leather (ASTM D1516)

Tongue Tear Strength (ASTM D1704)

Buckle Tear Strength (ASTM D1813)

Stitch Tear Strength, Single Hole (ASTM D4786)

Stitch Tear Strength, Double Hole (ASTM D1705)

Bursting Strength of Leather by the Ball Method (ASTM D2207)

Tensile Strength of Leather (ASTM D2209)

Breaking Strength of Leather by the Grab Method (ASTM 2208)

Elongation of Leather (ASTM 2211)

Water Absorption (Static) of Leather (ASTM D1815)

Permeability to Water Vapor (ASTM D5052)

Piping

Grain Cracking

Cold-Crack Resistance of Upholstery Leather (ASTM D1912)

Stiffness

Staining

Compressibility of Leather (ASTM 2213)

Crocking (ASTM D5053)

Fire Resistance of Leather

Corrosion Produced by Leather in Contact with Metal (ASTM

D1611)

Colorfastness and Transfer of Color in the Washing of Leather (ASTM D2096)

Flex Testing of Finish on Upholstery Leather (ASTM 2097)

Dynamic Water Resistance of Shoe Upper Leather by the Dow

Corning Leather Tester (ASTM 2098)

Dynamic Water Resistance of Shoe Upper Leather by the Maeser

Water Penetration Tester (ASTM 2099)

Resistance to Wetting of Garment-Type Leathers (Spray Test)

(ASTM D1913)

Grain Crack and Extension of Leather by the Mullen Test (ASTM 2210)

Slit Tear Resistance of Leather (ASTM D2212)

Estimating the Thermal Conductivity of Leather with the Cenco-

Fitch Apparatus (ASTM D2211)

Resistance of Chrome-Tanned White Shoe Upper Leather to

Artificial Perspiration (ASTM D2211)

Apparent Density of Leather (ASTM D2346)

Measuring the Relative Stiffness of Leather by Means of a

Torsional Wire Apparatus (ASTM D2821)

Measuring Break Pattern of Leather (Break Scale) (ASTM D2941)

Soak Waters - General

Lime Liquors - General

Bate Waters - General

Solids and Ash of Beamhouse Liquors 
F20

F21

F30

F35

F40

F50

F51

F52

F60

G1

G3

G4

G5

G6

H1

$\mathrm{H} 2$

H3

H4

H5

H6

H7

H8

H10

H15

H16

H17

H18

H20

H21

H22

$\mathrm{H} 23$

H30

H31

H32

$\mathrm{H} 40$

$\mathrm{H} 41$

H42

H43

H44

H45

H46

H47

H48

H49

H50

H52

H53

Total Volatile Nitrogen

Total Volatile Amine Nitrogen an Free Ammonia Analysis

Ammonia in Bate Waters

Total Caustic Alkalinity

Calcium in Beamhouse Liquors

Chlorides in Beamhouse Liquors

Sulfides in Lime Liquors

Sulfates in Beamhouse Liquors

$\mathrm{pH}$ Values of Beamhouse Liquors

Miscellaneous Tannery Materials - General

Egg Yolk

Lactic Acid

Oxalic Acid

Tannery Sugars

Fats, and Oils of Animal, Vegetable and Marine Origin - General

Hard Greases - General

Moellon - General

Compounded Oils - General

Sulfonated and Sulfated Oils (ASTM D500)

Commercial Soap and Soap Products

Sponging Compounds - General

Mineral Oil - General

Specifications for Reagents and Equipment

Specific Gravity of Oils and Liquid Fats (ASTM D5355)

Melting Point

Titer Test (ASTM 5565)

Cloud and Pout Point (ASTM D5551 and D5346)

Moisture and Volatile Matter (ASTM D5556)

Insoluble Impurities (ASTM D5557)

Ash (ASTM D5347)

Sediment in Moellon

Free Fatty Acids (ASTM D5555)

Saponification Value (ASTM D5558)

Iodine Value - Wijs Method (ASTM D5554)

Moisture (ASTM D5348)

Moisture and Volatile Matter (ASTM D5349)

Organically Combined Sulfuric Anhydride Titration Test (ASTM D5350)

Organically Combined Sulfuric Anhydride Extraction-Titration

Test (for Sulfated Oils) (ASTM D5351)

Organically Combined Sulfuric Anhydride Ash-Gravimetric Test

(in the Presence of True Sulfonates) (ASTM D5352)

Total Desulfated Fatty Matter (for Sulfated Oils) (ASTM D5353)

Total Active Ingredients (ASTM D5354)

Unsaponifiable Nonvolatile Matter (for Sulfated Oils) (ASTM D5553)

Inorganic Salts (H48) (ASTM D5566)

Total Alkalinity and Total Ammonia (ASTM D5564)

Acidity as Free Fatty Acids of Acid Number in the Presence of

Dark Colored Oils but in the Absence of Ammonium or

Triethanolamine Soaps (Brine Method) (ASTM 5559)

Acidity as Free Fatty Acids or Acid Number in the Presence of Ammonium or Triethanolamine Soaps (ASTM 5562)

Neutral Fatty Matter (ASTM D5560) 
Sampling Leather for Physical and Chemical Tests (ASTM

$\mathrm{J} 2$

$\mathrm{J} 10$

$\mathrm{J} 15$

$\mathrm{J} 25$

J30

$\mathrm{J} 40$

J50

$\mathrm{K} 1$

K5

$\mathrm{K} 10$

K11

$\mathrm{K} 12$

L1

$\mathrm{X} 1$
D2813)

Sampling Heavy Leather for Physical Tests (ASTM D2813)

Sampling of Vegetable Materials Containing Tannin

Sampling of Vegetable-Tanned Leathers (ASTM D2813)

Sampling of Mineral Tanned Leather for Chemical Tests (ASTM D2813)

Sampling of Beamhouse Liquors

Sampling of Tannery Chemicals

Sampling of Fats and Oils and Their Products

Total Solids and Ash in Leather Finish (ASTM D4906)

Nitrocellulose in Finish on Leather (ASTM D4907)

Flexibility and Adhesion of Finish on Leather

Tackiness of Finish on Leather (ASTM 4908)

Method for Testing Resistance of Colored Leather to Bleeding

(ASTM D5552)

The Resistance of Leather to the Growth of Fungi

Standards Definitions of Terms Relating to Leather 


\section{C.4 ASTM Textile Standards 44}

The following standards are categorized by the ASTM volume they appear in (either 07.01 or 07.02) and are listed numerically.

\section{VOLUME 07.01}
D $76-93$
D $123-93 a$
D $204-93$
D 276 - 87 (1993)
D $418-93$
D $434-75$
D $461-93$
D $519-90$
D $541-87$
D $578-90$
D $579-90$
D $580-89 a$
D $581-89$
D $584-90$
D $629-88$
D $681-87(1993)$
D 737-75 (1980)
D $861-89$
D 885 - 85 (1992)
D $885 \mathrm{M}-85$

D 1059 - 87 (1992)

D 1060 - 85 (1991)

D $1113-90 \mathrm{a}$

D $1117-80$

D $1230-94$

D 1233 - 88 (1993)

D 1234 - 85 (1990)

D 1244 - 81 (1991)

D 1282 - 89a

D 1283 - 85 (1990)

D $1284-87$

D 1294 - 94

D 1334 - 91
Specification for Tensile Testing Machines for Textiles.

Terminology Related to Textiles.

Methods of Testing Sewing Threads.

Test Methods for Identification of Fibers in Textiles.

Methods of Testing Pile Yarn Floor Covering Construction.

Test Method for Resistance to Slippage of Yarns in Woven

Fabrics Using a Standard Seam.

Test Methods for Felt.

Test Methods for Length of Fiber in Wool Top.

Specifications for Single Jute Yarn.

Specification for Glass Fiber Strands.

Specification for Greige Woven Glass Fabrics.

Specification for Greige Woven Glass Tapes and Webbing.

Specification for Glass Fiber Greige Braided Tubular Sleeving.

Test Method for Wool Content of Raw Wool - Laboratory Scale.

Test Methods for Quantitative Analysis of Textiles.

Specification for Jute Rove and Plied Yarn for Electrical and Packing Purposes.

Test Method for Air Permeability of Textile Fabrics.

Practice for Use of the Tex System to Designate Linear Density of

Fibers, Yarn Intermediates, and Organic-Base Fibers.

Methods of Testing Tire Cords, Tire Cords Fabrics, and Industrial

Filament Yarns Made from Man-Made, and Organic-Base Fibers.

Methods of Testing Tire Cords, Tire Cord Fabrics, and Industrial

Filament Yarns Made from Man-Made, and Organic-Base Fibers

[Metric].

Test Method for Yarn Number Based on Short-Length Specimens.

Practice for Core Sampling of Raw Wool in Packages for

Determination of Percentage of Clean Wool Present.

Test Method for Vegetable Matter and Other Alkali-Insoluble

Impurities in Scoured Wool.

Methods of Testing Non-woven Fabrics.

Test Method for Flammability of Apparel Textiles.

Specification for Twine Made from Bast and Leaf Fibers.

Method of Sampling and Testing Staple Length of Grease Wool.

Practice for Designation of Yarn Construction.

Test Method for Resistance to Airflow as an Indication of Average

Fiber Diameter of Wool Top, Cam, and Scoured Wool.

Test Method for Alkali-Solubility of Wool.

Test Methods for Relaxation and Consolidation Dimensional

Changes of Stabilized Knit Wool Fabrics.

Test Method for Tensile Strength and Breaking Tenacity of Wool

Fiber Bundles - 1-in. (25.4 mm Length).

Test Method for Wool Content of Raw Wool - Commercial Scale.

441994 Annual Book of ASTM Standards. pp. x-xiv. 1994. 
D 1335 - 67 (1972)

D 1336 - 72 (1977)

D 1388 - 64 (1975)

D $1422-92$

D $1423-92$

D $1424-83$

D $1425-89$

D $1440-90$

D 1441 - 87 (1993)

D $1442-93$

D $1445-90$

D 1447 - 89 (1994)

D $1448-90$

D $1464-90$

D $1518-85$

D $1574-87 a$

D 1575 - 90

D $1576-90$

D $1577-90$

D 1578 - 93

D 1683 - 90a

D $1684-90$

D 1770 - 88 (1993)

D $1774-93$

D $1775-90$

D 1776 - 90

D 1777 - 64 (1975)

D $1871-94$

D $1907-89$

D $1908-89$

D 1909 - 86 (1990)

D 2050 - 87 (1992)

D $2051-86(1991)$

D 2052 - 85 (1990)

D 2053 - 86 (1991)

D 2054 - 86 (1991)

D $2057-90$

D 2058 - 87 (1992)

D 2059 - 87 (1992)

D 2060 - 90

D $2061-93$

D 2062 - 87 (1992)
Test Method for Tuft Bind of Pile Floor Coverings.

Test Method for Distortion of Yam in Woven Fabrics.

Test Methods for Stiffness of Fabrics.

Test Method for Twist in Single Spun Yarns by the Untwist-

Retwist Method.

Test Method for Twist in Yarns by the Direct-Counting Method.

Test Method for Tear Resistance of Woven Fabrics by Falling

Pendulum (Elmendorf) Apparatus.

Test Method for Unevenness of Textile Strands Using Capacitance

Testing Equipment.

Test Method for Length and Length Distribution of Cotton Fibers

(Array Method).

Practice for Sampling Cotton Fibers for Testing.

Test Method for Maturity of Cotton Fibers (Sodium Hydroxide

Swelling and Polarized Light Procedures).

Test Method for Breaking Strength and Elongation of Cotton

Fibers (Flat Bundle Method).

Test Method for Length and Length Uniformity of Cotton Fibers

by Fibrograph Measurement.

Test Method for Micronaire Reading of Cotton Fibers.

Test Method for Differential Dyeing Behavior of Cotton.

Test Method for Thermal Transmittance of Textile Materials.

Test Method for Extractable Matter in Wool and Other Fibers.

Test Method for Fiber Length of Wool in Scoured Wool and in

Card Silver.

Test Method for Moisture in Wool by Oven-Drying.

Test Methods for Linear Density of Textile Fibers.

Test Method for Breaking Load of Skeins.

Test Method for Failure in Sewn Seams of Woven Fabrics.

Practice for Lighting Cotton Classing Rooms for Color Grading.

Test Method for Neps, Vegetable Matter, and Colored Fiber in

Wool Top.

Test Method for Elastic Properties of Textile Fibers.

Test Methods for Tension and Elongation of Wide Elastic Fabrics.

Practice for Conditioning Textile for Testing.

Method for Measuring Thickness of Textile Materials.

Test Methods for Adhesion of Single-Filament Steel Wire to

Rubber.

Test Method for Yarn Number by the Skein Method.

Test Method for Needle-Related Damage Due to Sewing in Woven Fabric.

Table of Commercial Moisture Regains for Textile Fibers.

Terminology Relating to Zippers.

Test Method for Durability of Finish of Zippers to Laundering.

Test Method for Colorfastness of Zippers to Dry-cleaning.

Test Method for Colorfastness of Zippers to Light.

Test Method for Colorfastness of Zipper Tapes to Crocking.

Test Method for Colorfastness of Zipper Tapes to Laundering.

Test Method for Durability of Finish of Zippers to Dry-cleaning.

Test Method for Resistance of Zippers to Salt Spray (Fog).

Methods for Measuring Zipper Dimensions.

Test Methods for Strength Tests for Zippers.

Test Methods for Operability of Zippers. 
D $2101-94$

D $2102-90$

D 2118 - 84 (1990)

D 2130 - 90

D 2165 - 90

D 2229 - $93 \mathrm{a}$

D 2252 - 85 (1991)

D $2253-88$

D $2255-90$

D 2256-90

D $2257-89$

D 2258 - 94

D $2259-91$

D 2260 - 89

D $2261-83$

D $2262-83$

D $2401-67$ (1972)

D $2402-90$

D $2462-90$

D 2475 - 88 (1993)

D $2494-94$

D 2495 - 87 (1993)

D $2497-80$

D 2524-91

D $2525-90$

D $2594-87$

D 2612 - 93a

D 2644 - 81 (1991)

D 2645 - 85 (1990)

D $2646-87$

D 2654 - 89a

D $2692-89$

D $2720-90$

D $2724-87$

D $2812-88$

D 2816 - 91
Test Methods for Tensile Properties of Single Man-Made Textile Fibers Taken from Yarns and Tows.

Test Method for Shrinkage of Textile Fibers.

Practice for Assigning a Standards Commercial Moisture Content for Wool and Its Products.

Test Method for Diameter of Wool and Other Animal Fibers by Microprojection.

Test Method for $\mathrm{pH}$ of Aqueous Extracts of Wool and Similar Animal Fibers.

Test Method for Rubber Property - Adhesion to Steel Cord.

Specification for Fineness of Types of Alpacea.

Test Method for Color of Raw Cotton Using the NickersonHunter Cotton Colorimeter.

Test Method for Grading Cotton Yarns for Appearance.

Test Method for Tensile Properties of Yarns by the Single Strand Method.

Test Method for Extractable Matter in Textiles.

Practice for Sampling Yarn for Testing.

Test Method for Shrinkage of Yarns in Boiling Water or Dry Heat.

Tables of Conversion Factors and Equivalent Yarn Numbers

Measured in Various Numbering Systems.

Test Method for Tearing Strength of Woven Fabrics by the

Tongue (Single Rip) Method (Constant-Rate-of-Extension Tensile

Testing Machine).

Test Method for Tearing Strength of Woven Fabrics by the

Tongue (Single Rip) Method (Constant-Rate-of-Extension Tensile

Testing Machine).

Test Method for Service Change of Appearance of Pile Floor

Coverings.

Test Method for Water Retention of Fibers (Centrifuge Method).

Test Method for Moisture in Wool by Distillation with Toluene.

Specification for Wool Felt.

Test Method for Commercial Mass of a Shipment of Yarn or ManMade Staple Fiber or Tow.

Test Method for Moisture in Cotton by Oven-Drying.

Tolerances for Man-Made Organic-Base Filament Single Yarns.

Test Method for Breaking Tenacity of Wool Fibers, Fl;at Bundle

Method - 1/8-in. (3.2 mm) Gage Length.

Practice for Sampling Wool for Moisture.

Test Methods for Stretch Properties of Knitted Fabrics Having

Low Power.

Test Method for Fiber Cohesion in Sliver and Top Static Tests.

Tolerances for Yarns Spun on the Woolen Systern.

Tolerances for Yarns Spun on the Cotton or Worsted Systems.

Test Methods for Backing Fabrics.

Test Methods for Moisture in Textiles.

Test Method for Air Wicking of Tire Fabrics, Tire Cord Fabrics,

Tire Cord, and Yarns.

Recommended Practice for Calculation of Commercial Weight and Yield of Scoured Wool, Top, and Notch for Various Commercial Compositions.

Test Methods for Bonded, Fused, and Laminated Apparel Fabrics. Test Method for Non-Lint Content of Cotton.

Test Method for Cashmere Coarse-Hair Content in Cashmere. 
D 2817 - 91

D 2859 - 93a

D 2904 - 91

D 2905 - 91

D 2906 - 91

D 2968 - 89

D 2969 - 92

D 2970 - 80

D 2970M - 80

D 3025 - 86

D $3106-89$

D 3107 - 75 (1980)

D $3108-89$

D 3135 - 87

D 3136 - 94

D $3181-89$

D 3217 - 94

D 3218 - 93

D $3333-90 a$

D $3374-89$

D 3412 - 89

D 3477 - 92

D $3511-82$

D 3512 - 82

D 3513 - 90

D 3514 - 81

D 3562 - 92

D 3597 - 94

D 3655 - 93
Specification for Maximum Cashmere Coarse-Hair Content in Cashmere.

Test Method for Flammability of Finished Textile Floor Covering Materials.

Practice for Inter-laboratory Testing of a Textile Test Method that Produces Normally Distributed Data.

Practice for Statements on Number of Specimens for Textiles.

Practice for Statements of Precision and Bias for Textiles.

Test Method for Med and Kemp Fiber in Wool and Other Animal

Fibers by Micro-projection.

Test Methods for Steel Tire Cords.

Method of Testing Tire Cords, Tire Cord Fabrics, and Industrial Yarns Made from Glass Filaments.

Method of Testing Tire Cords, Tire Cord Fabrics, and Industrial Yarns Made from Glass Filaments [Metric].

Practice for Standardizing Cotton Fiber Test Results by Use of Calibration Cotton Standards.

Test Method for Permanent Deformation of Elastomeric Yarns.

Test Method for Stretch Properties of Fabrics Woven from Stretch Yarns.

Test Method for Coefficient of Friction, Yarn to Solid Material. Specification for Performance of Bonded, Fused, and Laminated Apparel Fabrics.

Terminology for Permanent Care Labels for Consumer Textile and Leather Products Other Than Carpet and Upholstery.

Practice for Conducting Wear Testing on Textile Garments.

Test Methods for Breaking Tenacity of Man-Made Textile Fibers in Loop or Knot Configurations.

Specification for Polyolefin Monofilaments.

\section{VOLUME 07.02}

Practice for Sampling Man-Made Staple Fibers.

Specification for Vinyl-Coated Glass Yarns.

Test Method for Coefficient of Friction, Yarn to Yarn.

Performance Specification for Men's and Boy's Woven Dress

Shirt Fabrics.

Test Method for Pilling Resistance and Other Related Surface

Changes of Textile Fabrics : Brush Pilling Tester Method.

Test Method for Pilling Resistance and Other Related Surface

Changes of Textile Fabrics : Random Tumble Pilling Tester

Method.

Test Method for Overlength Fiber Content of Man-Made Staple

Fiber.

Test Method for Resistance of Apparel Fabrics to Pilling

(Elastomeric Pad Method).

Performance Specification for Men's and Women's Sliver Knitted

Overcoat and Jacket Fabrics.

Specification for Woven Upholstery Fabrics - Plain, Tufted, or Flocked.

Performance Specification for Men's and Women's Sliver Knitted Overcoat and Jacket Fabrics. 

D $3656-89$
D 3657 - 88 (1993)
D 3659 - 80 (1993)
D 3660 - 90
D $3661-90$
D 3690 - 78 (1990)
D 3691 - 78 (1990)
D 3692 - 89
D $3693-91$
D $3773-90$
D $3774-89$
D 3775 - 85 (1990)
D 3776 - 85 (1990)
D $3777-91$
D 3778 - 94
D 3779 - 81 (1990)
D 3780 - 94
D 3781 - 79 (1990)
D 3782 - 79 (1990)

D $3783-94$

D 3784 - 93

D $3785-92$

D $3786-87$

D $3817-89$

D 3818 - 92

D 3819 - 94

D 3820 - 94

D 3821 - 81 (1993)

D 3822 - 94

D $3823-94$

D 3882 - 90

D $3883-90$
Specification for Insect Screening and Louver Cloth Woven from Vinyl-Coated Glass Yarns.

Specification for Zipper Dimensions.

Test Method for Flammability of Apparel Fabrics by Semi-

Restraint Method.

Test Method for Staple Length of Man-Made Fibers, Average and Distribution (Fiber Array Method).

Test Method for Staple Length of Man-Made Fibers, Average and Distribution (Single-Fiber Length Machine Method).

Performance Specification for Vinyl-Coated and Urethane-Coated Upholstery Fabrics - Indoor.

Performance Specification for Woven, Lace, and Knit Household Curtain and Drapery Fabrics.

Practice for Selection of Zippers for Care-Labeled Apparel and Household Furnishings.

Specification for Labeled Length per Holder of Sewing Thread.

Test Methods for Length of Woven Fabric.

Test Methods for Width of Woven Fabric.

Test Method for Fabric Count of Woven Fabric.

Test Methods for Mass per Unit Area (Weight) of Woven Fabric.

Practice for Writing Specifications for Textile.

Performance Specification for Women's and Girls' Drycleanable

Woven Dress Coat Fabrics.

Performance Specification for Women's and Girls' Woven

Rainwear and All-Purpose Water-Repellent Coat Fabrics.

Performance Specification for Men's and Boy's Woven Dress Suit Fabric and Woven Sportswear Jacket, Slack, and Trouser Fabrics. Performance Specification for Men's and Boy's Knitted Rainwear and All-Purpose, Water-Repellent Coat Fabrics.

Performance Specification for Men's and Boy's Knitted Dress

Suit Fabrics and knitted Sportswear Jacket, Slack, and Trouser Fabrics.

Performance Specification for Woven Flat Lining Fabrics for Men's and Boy's Apparel.

Performance Specification for Woven Necktie and Scarf Fabrics. Test Method for Hydraulic Bursting Strength of Knitted Goods and Non-Woven Fabrics - Diaphragm Bursting Strength Tester Method.

Test Method for Bursting Strength of Knitted Goods - ConstantRate-of-Traverse (CRT) Ball Burst Test.

Test Method for Maturity Index of Cotton Fibers by Fibrograph. Test Method for Linear Density and Maturity Indices of Cotton Fibers (IIC-Shirley Fineness/Maturity Test).

Performance Specification for Men's and Boys' Woven Pajama Fabrics.

Performance Specification for Men's and Boy's Woven Underwear Fabrics.

Performance Specification for Woven Terry Household Kitchen and Bath Towel Fabrics.

Test Method for Tensile Properties of Single Textile Fibers.

Practice for Determining Ticket Numbers for Sewing Threads.

Test Method for Bow and Skewness in Woven and Knitted

Fabrics.

Test Method for Yarn Crimp or Yarn Take-up in Woven Fabrics. 
D 3884 - 92

D 3885 - 92

D 3886 - 92

D 3887 - 94

D 3888 - 90

D 3936 - 80

D 3937 - 90

D 3938 - 93

D 3939 - 93

D 3940 - 83

D 3990 - 93

D 3991 - 85 (1991)

D 3992 - 85 (1991)

D 3993 - 81 (1990)

D 3994 - 94

D 3995 - 92

D 3996 - 92

D 4028 - 84

D 4029 - 90

D 4030 - 89

D 4031 - 81 (1987)

D 4032 - 94

D 4033-92

D 4034 - 92

D 4035 - 92

D 4036 - 81 (1990)

D 4037 - 81 (1990)

D 4038 - 94

D 4109 - 92

D $4110-92$

D $4111-92$
Test Method for Abrasion Resistance of Textile Fabrics (Rotary Platform, Double-Head Method).

Test Method for Abrasion Resistance of Textile Fabrics (Flexing and Abrasion Method).

Test Method for Abrasion Resistance of Textile Fabrics (Inflated Diaphragm Method).

Specification for Knitted Fabrics.

Definition of Terms Relating to Open-End Spinning.

Test Method for Delamination of Strength of Secondary Backing of Pile Floor Coverings.

Test Method for Crimp Frequency of Man-Made Staple Fibers.

Guide for Evaluation of Textile Products in Relation to

Refurbishing Described on Care labels.

Test Method for Snagging Resistance of fabrics (Mace Test Method).

Test Method for Bursting Strength (Load) and Elongation of Sewn Seams of Knit or Woven Stretch Textile Fabrics.

Terminology Relating to Fabric Defects.

Specifications for Fineness of Wool or Mohair and Assignment of Grade.

Specifications for Fineness of Wool Top or Mohair Top and Assignment of Grade.

Performance Specification for Woven, Thermal, Flocked, Nonwoven, and Knitted Household Blanket Fabric.

Performance Specification for Men's, Women's, and Children's

Woven Swimwear Fabrics.

Performance Specification for Men's and Women's Knitted Career Apparel Fabrics: Dress and Vocational.

Performance Specification for Men's, Women's, and Children's

Knit Swimwear Fabrics.

Specification for Solar Screening Woven from Vinyl-Coated Fiber Glass Yarn.

Specification for Finished Woven Glass Fabrics.

Specification for Glass Fiber Cord and Sewing Thread.

Test Method for Bulk Properties of Textured Yarns.

Test Method for Stiffness of Fabric by the Circular Bend

Procedure.

Test Method for Determining Yarn Slippage in Sewn Seams Made from Upholstery Fabrics - Plain, Tufted, or Flocked.

Test Method for Determining Yarn Slippage in Sewn Seam in Woven Upholstery Fabrics - Plain, Tufted, or Flocked.

Performance Specification for Knitted Necktie and Scarf Fabrics.

Performance Specification for Woven and Knit Household

Pillowcase, Bed Sheet and Crib Sheet Fabrics.

Performance Specification for Woven, Knitted, or Flocked,

Bedspread Fabrics.

Performance Specification for Women's and Girl's Woven Dress and Blouse Fabrics.

Performance Specification for Men's and Boy's Woven Coverall, Dungaree, Overall, and Shop Coat Fabrics.

Performance Specification for Men's and Boys' Knitted Bathrobe, Dressing Gown, and Pajama Fabrics.

Performance specification for Woven Napery and Tablecloth

Fabrics: Household and Institutional. 

D 4112 - 92
D 4113 - 92
D 4114 - 92 a
D 4115 - 92
D $4116-92$
D 4117 - 92
D $4118-92$
D 4119 - 92
D $4120-93$
D 4151 - 92
D 4152 - 82
D $4153-82$
D 4154- 92
D 4155-92
D $4156-92$
D 4157- 92
D 4158- 92
D 4231 - 83 (1989)
D $4232-92$
D $4233-92$
D $4234-92$
D 4235 - 92
D 4238 - 90
D 4268 - 83
D 4270 - 90
D 4271 - 88 (1993)
D 4272 - 93
D 4389 - 89
D 4390 - 93
D $4391-93 a$

Performance Specification for Woven Umbrella Fabrics.

Performance Specification for Woven Slipcover Fabrics.

Performance Specification for Woven Flat Lining Fabrics for

Women's and Girls' Apparel.

Performance Specification for Women's and Girls' Knitted and

Woven Dress Glove Fabrics.

Performance Specification for Women's and Girls' Knitted and

Woven Corset-Girdle-Combination Fabrics.

Performance Specification for Women's and Girls' Woven Robe

Negligee, Nightgown, Pajama, Slip, and Lingerie Fabrics.

Performance Specification for Women's Woven Coverall,

Dungaree, Overall and Shop Coat Fabrics.

Performance Specification for Men's and Boys' Knitted Dress

Shirt Fabrics.

Test Method for Fiber Cohesion in Roving, Sliver, and Top

(Dynamic Tests).

Test Method for Flammability of Blankets.

Performance Specification for Woven Institutional Dish, Huck, and Terry Bath Towel Fabrics.

Performance Specification for Men's, Women's and Children's

Woven Handkerchief Fabrics.

Performance Specification for Men's and Boy's Knitted and

Woven Beachwear and Sport Shirt Fabrics.

Performance Specification for Women's and Girls' Woven

Sportswear, Shorts, Slacks, and Suiting Fabrics.

Performance Specification for Women's and Girls' Knitted

Sportswear Fabrics.

Test Method for Abrasion Resistance of Textile Fabrics

(Oscillatory Cylinder Method).

Test Method for Abrasion of Textile Fabrics (Uniform Abrasion

Method).

Practice for Evaluation of Men's and Boys' Home Launderable

Woven Dress Shirts and Sport Shirts.

Performance Specification for Men's and Women's Dress and

Vocational Career Apparel Fabrics.

Performance Specification for Women's and girl's Knitted and

Woven Brassiere Fabrics.

Performance Specification for Wornen's and Girls' Knitted Robe, Negligee, Nightgown, Pajama, Slip, and Lingerie Fabrics.

Performance Specification for Women's and Girls' Knitted Blouse and Dress Fabrics.

Test Method for Electrostatic Propensity of Textiles.

Methods of Testing Fiber Ropes.

Guide for Using Existing Practices in Developing Test Methods for Textiles.

Practice for Writing Statements on Sampling in Test Methods for Textiles.

Specification for Flame-Resistant Materials Used in Camping

Tentage.

Specification for Finished Glass Fabrics Woven from Rovings.

Practice for the Evaluation of the Performance of Terry Bathroom

Products for Household Use.

Terminology Relating to the Burning Behavior of Textiles. 

D 4393 - 94
D 4465 - 85 (1990)
D $4466-85$
D 4467 - 94
D 4510 - 93
D 4522 - 86 (1993)
D 4523 - 85 (1993)
D 4524 - 86 (1993)
D 4604 - 86
D 4605 - 86
D 4685 - 87
D 4686 - 91
D 4697 - 91
D 4720 - 87 (1994)
D 4721 - 89 (1994)
D 4723 - 90 (1993)
D 4724 - 87 (1992)
D 4769 - 88 (1994)
D 4770 - 88
D 4771 - 94
D 4772 - 88
D 4776 - 88
D 4777 - 88
D 4845 - 89
D $4846-88$
D 4847 - 88
D 4848 - 94a
D 4850 - 91
D 4851 - 88
D 4852 - 88 (1994)
D 4853 - 91
D 4854 - 91
D 4855 - 91
Test Method for Strap Peel Adhesion of Reinforcing Cords or Fabrics to Rubber Compounds.
Performance Specification for Zippers for Denim Dungarees.
Terminology for Multicomponent Textile Fibers.
Practice for Inter-laboratory Testing of a Textile Test Method That Produces Non-Normally Distributed Data.
Test Method for Counting Partial Cleavages in Wool and Other Animal Fibers.
Performance Specification for Feather-Filled and Down-Filled
Products.
Terminology Relating to Feather-Filled and Down-Filled
Products.
Test Method for Composition of Plumage.
Test Methods for Measurement of Cotton Fibers by High Volume Instruments (HVI) (Motion Control Fiber Information System).
Test Methods for Measurement of Cotton Fibers by High Volume Instruments (HVI) (Special Instrument Laboratory System).
Test Method for Pile Retention of Corduroy Fabrics.
Guide for Identification of Frequency Distributions.
Guide for Maintaining Test Methods in the User's Laboratory.
Practice for Evaluation of the Performance of Soft Window
Coverings.
Practice for Evaluation of the Performance of Machine Washable and Drycleanable Bed Coverings and Accessories.
Index and Descriptions of Textile Heat and Flammability Test
Methods and Performance Specifications .
Test Methods for Degree of Filament Yarn Entanglement by
Needle Insertion Methods.
Performance Specification for Woven and Warp Knitted
Comforter Fabrics.
Test Method for Evaluation of Man-made Fiber Batting Used as
Filling in Outerwear Apparel.
Performance Specification for Knitted Upholstery Fabrics for Indoor Furniture.
Test Method for Surface Water Absorption of Terry Fabrics
(Water-Flow Test Method).
Test Method for Adhesion of Tire Cords and Other Reinforcing
Cords to Rubber Compounds by H-Test Procedure.
Test Method for Adhesion of Tire Cords and Other Reinforcing
Cords to Rubber Compounds by Hot U-Test Procedure.
Terminology Relating to Wool.
Test Method for Resistance to Unsnapping of Snap Fasteners.
Performance Specification for Woven Awning and Canopy
Fabrics.
Terminology Relating to Tensile Properties of Textiles.
Terminology Relating to Fabric and Related Terms.
Test Method for Coated and Laminated Fabrics for Architectural
Use.
Practice for Evaluation of Attached Upholstery Fabrics.
Guide for Reducing Test Variability.
Guide for Estimating the Magnitude of Variability from Expected
Sources in Sampling Plans.
Practice for Comparing Test Methods. 

D $4909-89$
D 4910 - 89
D 4911 - 94
D $4912-89$
D $4920-89$
D $4963-89$
D $4964-94$
D $4965-89 b$
D 4966 - 89
D 4970 - 89
D 4974 - 93
D 4975 - 93
D $5034-90$
D 5035 - 90
D 5038 - 90
D $5103-90$
D $5104-90$
D $5169-91$
D 5170-91
D $5171-91$
D $5219-94 b$
D $5328-92$
D $5251-92$
D 5252 - 92
D $5253-92$
D 5278 - 92
D 5332- 92
D 5344 - 93
D 5362 - 93
D 5478 - 93
D 5417 - 93
Test Method for Color Stability of Vinyl-Coated Glass Textiles to Accelerated Weathering.
Standard Table of Body Measurements for Infants, Ages 0 to 18 Months.
Tolerances for Man-Made Yarns Spun on the Parallel Worsted or Modified Worsted System.
Test Method for Fabric Stability of Vinyl-Coated Glass Yarn
Insect Screening and Louver Cloth.
Terminology Relating to Moisture in Textiles.
Test Method for Ignition Loss of Glass Strands and Fabrics.
Test method for Tension and Elongation of Elastic Fabrics
(Constant-Rate-of-Expansion Type Tension Testing Machine).
Terminology of Seam Finishes in Home Sewing.
Test Method for Abrasion Resistance of Textile Fabrics
(Martindale Abrasion Tester Method).
Test Method for Pilling Resistance and Other Related Surface
Changes of Textile Fabrics (Martindale Pressure Tester Method).
Test Method for Thermal Shrinkage of Yarn and Cord Using the
Textile Thermal Shrinkage Oven.
Test Methods for Single-Filament Tire Bead Wire Made from
Steel.
Test Method for Breaking Force and Elongation of Textile Fabrics (Grab Test).
Test Method for Breaking Force and Elongation of Textiles
Fabrics (Strip Test).
Terminology of Textile Conversation.
Test Method for Length and Length Distribution of Man-Made
Staple Fibers (Single-Fiber Test).
Test Method for Shrinkage of Textile Fibers (Single-Fiber Test).
Test Method for Shear Strength (Dynamic Method) of Hook and
Loop Touch Fasteners.
Test Method for Peel Strength ("T" Method) of Hook and Loop
Touch Fasteners.
Test Method for Impact Resistance of Plastic Sew-Through Flange
Buttons.
Terminology Relating to Body Dimensions for Apparel Sizing.
Test Method for Smoldering Combustion Potential of Cotton-
Based Batting.
Practice for the Operation of the Tetrapod Walker Drum Tester.
Practice for the Operation of the Hexapod Drum Tester.
Terminology of Writing Care Instructions and General
Refurbishing Procedure for Textile Floor Coverings and Textile Upholstered.
Test Method for Elongation of Narrow Elastic Fabrics (Bean Bag Test Method).
Test Method for Fiber Length and Length Distribution of Cotton and Man-Made Staple Fibers.
Test Method for Extension Force of Partially Oriented Yarn.
Test Method for Snagging Resistance of Fabrics (Bean Bag Test
Method).
Performance Specification for Woven and Knitted Shower
Curtains for Institutional and Household Use.
Practice for the Operation of the Vettermnan Drum Tester. 
D 5426 - 93

D $5427-93$

D $5428-93$

D $5429-93 a$

D $5430-93$

D $5431-93$

D 5432 - 93

D 5433 - 93

D 5446 - 93

D $5489-93$

D $5497-94$

D 5585 - 93

D 5586 - 94
Practice for the Visual Inspection and Grading of Fabrics Used for Inflatable Restraints.

Practice for the Accelerated Aging of Inflatable Restraint Fabrics. Practice for Evaluating the Performance of Inflatable Restraint Modules.

Practice for the Pre-treatment of Backing Fabrics Used in Textile Conservation Research.

Test Methods for Visually Inspecting and Grading Fabrics.

Performance Specification for Woven and Knitted Sheeting Products for Institutional and Household Use.

Performance Specification for Blanket Products for Institutional and Household Use.

Performance Specification for Towel Products for Institutional and Household Use.

Test Methods for Determining Physical Properties of Fabrics Used in Inflatable Restraints.

Guide for Care Symbols for Permanent Care Labels On Consumer Textile Products.

Terminology Relating to Buttons.

Standard Table of Body Measurements for Adult Female Misses Figure Type Size 2-20.

Standard Tables of Body Measurements for Women Aged 55 and Older (All Figure Types). 


\section{C.5 Government/Military Standards and Specifications ${ }^{45}$}

Because of its size and diversity of content, the military and federal standards and specifications are organized into two levels of subjects. Standards within each subgroup are listed numerically. This section is broken into the following groups and subgroups:

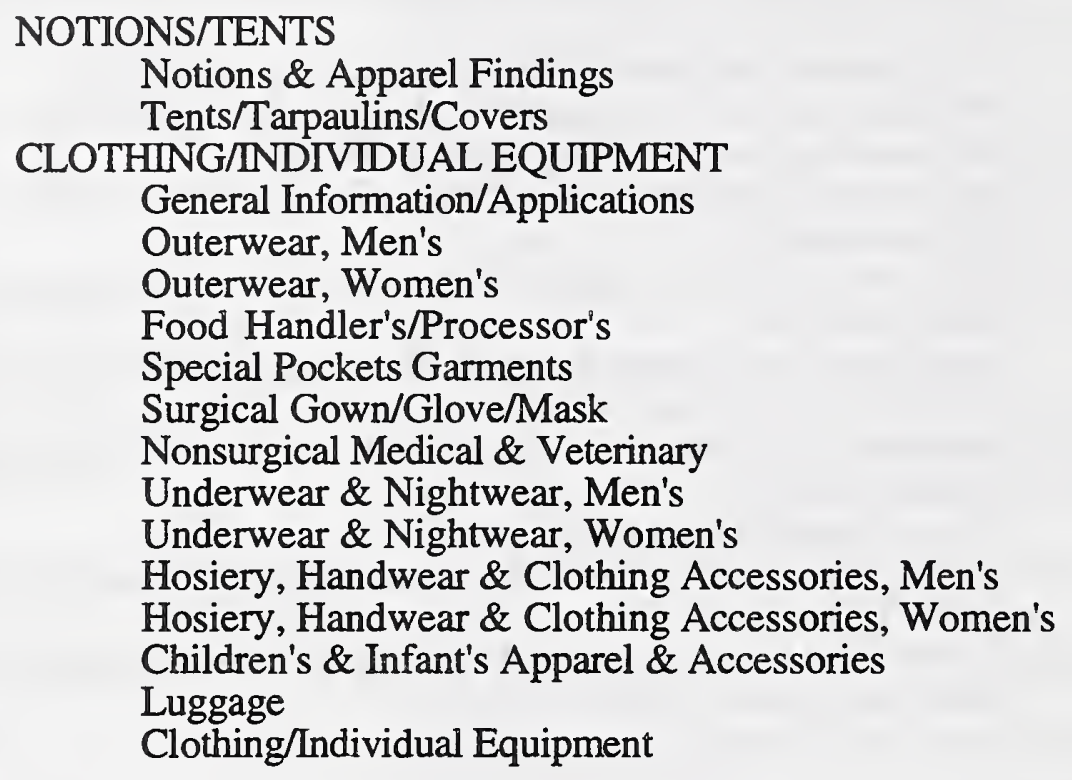

\section{NOTIONS/TENTS}

Notions \& Apparel Findings KSC-SPEC-P-0016 REV A

DDD-L-20F (1)

A-A-119B
MIL-HDBK-150B
FF-N-180A INT AMD 1
GGG-N-202C Valid Notice 1
MIL-B-286D
V-B-871F
MIL-STD-1394B
A-A-1749
MIL-B-1860E
MIL-B-1963J (1)
MIL-S-3276H
MIL-S-3577G
MIL-B-14656
MIL-L-15040F Valid Notice 1
MIL-P-15064G (1)
MIL-C-15065J
MIL-L-17507F (1)
MIL-E-17568C

Minimum Requirements for Garment Snap Fastener, Specification for. FSC PACK

Label: for Clothing, Equipage, and Tentage, (General Use). FSC 8315

Pin, Safety. FSC 8315

Clothing Components for Military Uniforms. FSC 8315

Needles, Except Surgical, Hand. FSC 8315

Needle, Sailmaker. FSC 8315

Buttons, Tack; and Tack, Button. FSC 8315

Button, Sewing Hole, and Button, Staple, (Plastic). FSC 8315

Provisions for Evaluating Quality of Cap Crowns. FSC 8405

Headband, Sweat. FSC 4240

Buckle, Slide, Plastic. FSC 8315

Buckles; and Clips, End, Strap (for Belt, Trousers). FSC 8315

Sewing Kits. FSC 8315

Sweatband, Headwear, Leather. FSC 8405

Buckle and Catch, Ceremonial, Army. FSC 8315

Label, Garment (Woven, Rayon). FSC 8315

Pads, Shoulder and Sleeve-Head. FSC 8315

Coat Fronts. FSC 8315

Lace, Ornamental. FSC 8315

Embroidery Materials, Metallic and Synthetic Metallic. FSC 8315

45 Information Handling Services. pp. 327-335, 340-434. 1994. 


$\begin{array}{ll}\text { MLL-F-17619E (1) } & \text { Frame, Service Cap (Man's). FSC 8405 } \\ \text { MLL-C-17620F } & \text { Crown, Service Cap (Man's). FSC 8405 } \\ \text { MLL-B-17910D } & \text { Buckle, Brass: for Belt, Coat, Man's. FSC 8315 } \\ \text { MIL-C-18186D (1) } & \text { Crowns, Service Cap. FSC 8405. } \\ \text { MIL-F-20268G } & \text { Frame, Cap, Man's. FSC 8405 } \\ \text { MLL-B-20269E } & \text { Buckles: Insignia and Plain. FSC 8315 } \\ \text { MIL-L-20271C Valid Notice } 1 & \text { Lace, Gold: Ornamental. FSC 8315 } \\ \text { MIL-B-20588F } & \text { Buckle, Center Bar (Military Police Belt). FSC 8315 } \\ \text { ML-S-22760C } & \text { Support Crown, Service Cap; and Support Holder. FSC 8405 } \\ \text { MIL-C-23486B } & \text { Collar, Coat, Man's: Polyester/Wool, Gabardine, Blue. FSC } \\ & \text { 8315 } \\ \text { MS35901 } & \text { Notions and Apparel Findings FSC Class 8315. FSC 8315 } \\ \text { MIL-B-40006D } & \text { Buckle, General Officers' Belt, Gold Plated. FSC 8315 } \\ \text { MIL-B-40092D } & \text { Braid, Textile, Cord-Edge, Polyester. FSC 8315 } \\ \text { MIL-F-43514B } & \text { Fastener, Plastic, for Equipage Items. FSC 8465 } \\ \text { MIL-S-43993C } & \text { Sweatband, Headwear: Artificial Leather. FSC 8405 } \\ \text { A-A-52067 } & \text { Binding, Textile, Cotton, Bias-Cut. FSC 8315 } \\ \text { A-A-55066 } & \text { Needles, Except Surgical, Hand. FSC 8315 } \\ \text { A-A-55187 } & \text { Braid, Textile (Flat). FSC 8315 } \\ \text { A-A-55190 } & \text { Sewing Kit. FSC 8315 } \\ \text { MIL-C-82114A } & \text { Coat Front: for Coats, Musicians. FSC 8315 } \\ \text { MIL-N-87224 Valid Notice 1 } & \text { Neck Tab, Women's, Shirts. FSC 8445 }\end{array}$

Tents/Tarpaulins/Covers

K-P-146E INT AMD 1

MIIL-P-500H

MII-P-501P

MIL-P-549K

MIL-P-608K

MIL-T-1110F

MII-T-1111G (2)

MIL-F-1461H

MIL-S-1484E

MIL-T-1712T

MIL-P-1716H (1)

MIL-S-1743H

MIL-T-1926G

MIL-T-1956D (1)

MIL-P-2383H

MIL-S-3725E Valid Notice 1

MUL-T-7249B Valid Notice 1

MIL-T-10009H

MUL-T-10035K

MIL-T-10069G (2)

MIL-T-10168J

MIL-I-10901H Valid Notice 1 MIL-U-11224E (1)
Frame, Service Cap (Man's). FSC 8405

Crown, Service Cap (Man's). FSC 8405

Buckle, Brass: for Belt, Coat, Man's. FSC 8315

Frame, Cap, Man's. FSC 8405

Lace, Gold: Oria and Plain. FSC 8315

Buckle, Center Bar (Military Police Belt). FSC 8315

Support Crown, Service Cap; and Support Holder. FSC 8405

Collar, Coat, Man's: Polyester/Wool, Gabardine, Blue. FSC

Buckle, General Officers' Belt, Gold Plated. FSC 8315

Braid, Textile, Cord-Edge, Polyester. FSC 8315

Fastener, Plastic, for Equipage Items. FSC 8465

Binding, Textile, Cotton, Bias-Cut. FSC 8315

Needles, Except Surgical, Hand. FSC 8315

Braid, Textile (Flat). FSC 8315

Coat Front: for Coats, Musicians. FSC 8315

Neck Tab, Women's, Shirts. FSC 8445

Tarpaulins, Cotton Duck, FWW/MR. FSC 8340

Plates, Tent, Peak and Ridge. FSC 8340

Pin, Tent, Metal. FSC 8340

Poles, Tent, Upright and Ridge. FSC 8340

Pole Section, Tent: Upright and Adapter, Tent Pole. FSC 8340

Tent, Assembly, M-1942. FSC 8340

Tent, Command Post, M-1945, Fire, Water, Weather and Mildew

Resistant, Olive Drab, Complete. FSC 8340

Frame Sections, Tent, Maintenance. FSC 8340

Shields, Stovepipe, Tent. FSC 8340

Tent, General Purpose, Medium. FSC 8340

Pole, Tent, Telescopic, Adjustable 5 Feet to 9 Feet, Magnesium.

FSC 8340

Slips, Tent Line. FSC 8340

Tent, Mountain, Two-Man, Complete with Pins and Poles. FSC 8340

Tarpaulins, Waterproof, Special Purpose, 10 Feet Long by 8 Feet Wide. FSC 8340

Pins, Tent, Wood. FSC 8340

Shelter Half, Tent. FSC 8305

Tarpaulin, Light Weight. FSC 8340

Tent, Kitchen, Flyproof, M-1948. FSC 8340

Tent, Hexagonal, Light Weight, M-1950. FSC 8340

Tent, Maintenance Shelter, Fire, Water, Weather, and Mildew

Resistant, Olive Drab. FSC 8340

Tent, Frame-Type, Insulated, Sectional, with Floor, 16 Feet

Wide, M1948, Complete. FSC 8340

Insect Bar: Field Type, Nylon Netting. FSC 7210

Umbrella, Surveyor's (Six-Rib). FSC 8340
Crowns, Service Cap. FSC 8405. 
MIL-T-12354F (1)

MIL-T-1219F

MIL-C-13489D

MIL-T-14038K

MII-T-14056G

MIL-C-18680C

MIL-C-22043

MIL-T-40001E

MU-T-40031E

MIIL-F-40132G

MII-P-40148F

MIL-T-41810K

MIL-T-41812H

MIL-T-41813F

MIL-T-41830E

MIL-S-43176C

MIL-T-43182D

MIL-T-43309C Valid Notice 1 MIL-T-43333C

MIL-T-43389 (1)

MIL-P-43413D

MIL-T-43416C

MII-T-43492C

MIL-T-43512B (1)

MIL-F-43695B

MIL-T-43764A

MIL-T-44222A

ML-T-44243A (1)

MIL-F-44251A

ML-T-44271A

MIL-F-44397 (1)

MIL-T-44400 (1)

MII-P-44403

MIL-C-44404

MIL-C-44413

MIIL-F-44425

MIL-T-44427
Tent, Arctic, 10 Man. FSC 8340

Tent Liner, General Purpose, Medium. FSC 8340

Cover and End Curtains; Cargo Body (for Military Vehicles). FSC 2540

Tent, General Purpose, Large. FSC 8340

Tent Liner, General Purpose, Large, Fire, Water, and Mildew

Resistant. FSC 8340

Fly, Tent: Fire, Water, Weather and Mildew Resistant. FSC 8340

Covers, Coated, Nylon (for Naval Ordnance Equipment). FSC

10GP

Tent, Observing, Triangulation, Ground Type, Complete with

Frame. FSC 8340

Tent, Observing, Astronomic, Complete with Fly and Frame.

FSC 8340

Frame Sections, Tent, Maintenance, Medium, Light Metal. FSC 8340

Poles, Tent, Telescopic, Adjustable, Aluminum. FSC 8340

Tent, General Purpose, Small. FSC 8340

Tent, Liner Sections, Frame-Type, Maintenance, Medium. FSC 8340

Tent Sections, Frame Type, Maintenance, Medium. FSC 8340

Tent, Vehicle Maintenance, Complete with A-Frame. FSC 8340

Screen, Latrine, Fire, Water, Weather, and Mildew Resistant

Treated, O.D. FSC 8340

Tent, Missile System Equipment Console (HAWK). FSC 8340

Tarpaulin: Cotton Duck for Wind Measuring Set. FSC 8340

Tent Liner, General Purpose, Small and Arctic, 10 Man. FSC 8340

Tarpaulin, Cotton Duck, Olive Drab No. 7; 20 Feet by 20 Inches.

FSC 8340

Poles, Tent, Light Metal, Special. FSC 8340

Tent, Sunshield, Theodolite. FSC 8340

Tent Sections, Frame Type, Expandable. FSC 8340

Tents, Missile System Equipment Console, (High-Power

Illuminator HAWK). FSC 8340

Frame Sections, Tent, Frame Type, Expandable. FSC 8340

Tents, Cable Splicer. FSC 8340

Tent, Liner Sections; Insulated (Temper). FSC 8340

Tent Sections, Tent, Extendable, Modular, Personnel (Temper).

FSC 8340

Frame Sections, Tent, Extendable, Modular, Personnel (Temper). FSC 8340

Tents, Extendable, Modular, Personnel (Temper), Assembly

Components. FSC 8340

Frame, Tent, (SICPS). FSC 8340

Tent, Fabric Assemblies, Standardized Integrated Command Post System. FSC 8340

Passageway, Complexing Kit. FSC 8340

Command Post, Tent, Standardized Integrated Command Post

System. FSC 5410

Cover, Nuclear, Biological, and Chemical Protective (NBC-CP).

FSC 8340

Frame Section, Tent, Five Soldier Crew. FSC 8340

Tent, Five Soldier Crew. FSC 8340 
MS5123 REV C Valid Notice 1 Cover, Fitted, Vehicular Body - Top. FSC 2540

A-A-55235

MIL-S-55507E (2)

MIL-S-55557A Notice 2

MIIL-T-82120A (1)

MIL-T-82152B

MIL-T-82288B

MIL-T-83788

MIL-C-83991A
Tarpaulin; Cotton Duck, Camouflage Green 483; 20 Feet by 20 Inches. FSC 8340

Shelter, Electrical Equipment (With or Without Equipment), Packaging of. FSC PACK

Shelter, Electrical Equipment S-330()/TRC-117(V). FSC 5410 Tarpaulins: Duck, Cotton; Fire, Water, Weather and Mildew Resistant Treated; with Carrying Bag. FSC 8340 Tarpaulins: Duck, Cotton, Vinyl Resin Coated Both Sides, 14 Feet Long by 6 Feet Wide. FSC 8340

Tarpaulin: Laminated, Vinyl-Nylon, Flexible. FSC 8340 Tent, Pyramidal, Survival, 3-4 Man, SRU-1/P. FSC 8340 Cover, Polyethylene, Pallet, Cargo HCU-6/E And HCU-12/E (Use A-A-55437). FSC 3990

\section{CLOTHING/INDIVIDUAL EQUIPMENT}

General Information/ Applications

MIL-HDBK-156

MII-STD-284A

MIL-L-35078M SUPP 1

MIL-C-44192A
Glossary of Military Clothing Fabrication Terms. FSC 8430

Visual Inspection Guide for Rubber Footwear. FSC 8430

Loads, Unit: Preparation of Semiperishable Subsistence Items:

Clothing, Personal Equipment and Equipage; General

Specification for. FSC PACK

Container, Shipping and Storage, Coat (Hanger Pack). FSC 8115
Outerwear, Men's

BBB-C-0050

MIL-STD-657A

MIL-C-8131E (1)

MII-STD-901B

BBB-S-1268B Valid Notice 1

BBB-S-1269B (2)

MIL-STD-1391D

MIL-STD-1488G

MII-STD-1492C

MII-STD-1494B

A-A-1626

A-A-1782

A-A-1783

A-A-1784

A-A-1785

A-A-1786

MIIL-C-1911J INT AMD 2

MIIL-S-2036J

MIIL-C-2202H

MII-O-2414H

MIL-T-2423L

MIL-S-3003K (1)

MIIL-S-3007J (1)
Cap, Softball. FSC 8415

Provision for Evaluating Quality of Service Caps. FSC 8405

Cap, Utility: Cotton, Sateen, Green. FSC 8405

Provisions for Evaluating Quality of Caps, Garrison, Men's. FSC 8405

Sweat Shirt. FSC 8415

Sweat Pants. FSC 8415

Provisions for Evaluating Quality of Overcoats, Men's. FSC 8405

Provisions for Evaluating Quality of Coats, Men's Dress. FSC 8405

Provisions for Evaluating Quality of Men's Shirts. FSC 8405

Provisions for Evaluating Quality of Raincoats. FSC 8405

Shirt, Man's and Women's (Long or Short Sleeve). FSC 8405

Cap, Civilian, Uniform. FSC 8405

Shirt, Man's (and Woman's; Long Sleeve). FSC 8415

Trousers, Man's (and Woman's - Summer Weight). FSC 8415

Trousers, Man's and Woman's (Winter Weight). FSC 8405

Shirt, Man's (and Woman's; Short Sleeve). FSC 8415

Cap, Camouflage Pattern. FSC 8415

Scarf, Neckwear, Wool. FSC 8440

Coveralls, Men's Cotton, Sateen. FSC 8405

Overcoat, Man's, Enlisted. FSC 8405

Trousers, Men's (White). FSC 8405

Poncho, Wet Weather, Heavy Duty. FSC 8405

Sweater, Man's, Olive Drab. FSC 8405 
MIL-C-3095G

MIL-C-3261F

MIL-H-3364D

MIL-S-3649F Valid Notice 1

MIL-J-7448K

MIL-S-10858H
Cap, Service, Man's, Air Force. FSC 8405

Cap, Garrison, Man's Air Force, Blue. FSC 8405

Helmet, Sun. FSC 8415

Shirt, Man's; Long Sleeve. FSC 8405

Jacket, Utility L-2B. FSC 8405

Shirt, Cold Weather, Field, Wool/Nylon, Olive Green 108. FSC 8415

MIL-C-13998H Valid Notice 1 Cap, Service, Wool. FSC 8405

MIL-C-15065J

MIL-C-16472H

MIL-C-17614F

MIL-S-17615E

MIL-S-17618H

MIL-H-19448C Valid Notice 1

MIL-C-19519G (1)

MIL-S-19984E

MIL-C-21083C (1)

MIIL-S-21088C Valid Notice 1

MIL-T-21704F

MIL-J-21708G

MIL-C-24918B

MIL-C-24920A

MIL-S-24922

MIL-C-24937A

MII-S-24950

MIIL-H-25754B (1)

MIL-C-27438G

Coat Fronts. FSC 8315

Cap, Knit (Watch). FSC 8405

Cap, Garrison, Man's. FSC 8405

Strap, Chine (Navy and Coast Guard). FSC 8405

Shirt, Man's, (Polyester/Cotton, Tropical, Short Sleeve).

Hat, Service: with Chin Strap. FSC 8405

Coat, Man's: Polyester/Wool, Gabardine; Blue. FSC 8405

Shirt, Man's: Khaki; with Quarter Length Sleeve. FSC 8405

Coat, Man's: Service, Officers, USMC. FSC 8405

Coast, Man's: White; Dress (Officer's). FSC 8405

Trousers, Cold Weather. FSC 8415

Jacket, Cold Weather. FSC 8415

Coat, All-Weather, Man's, W/Removable Liner. FSC 8405

Coat, All-Weather, Man's, with Removable Liner. FSC 8405

Sweater, Man's (Flame Retardant). FSC 8405

Cap, Combination, Man's, (CG). FSC 8405

Shirt, Man's, Dress White, Long Sleeve (CG). FSC 8405

Hood, Winter, Knit, Wool. FSC 8415

Coat, Men's, Service. FSC 8405

Coveralls, Men's CMU-3/P. FSC 8405

Trousers, Men's: Musicians. FSC 8405

MIL-T-28902B

MIL-T-28919 Valid Notice 1

MIL-T-28920A Valid Notice 1

MIL-V-28936 Valid Notice 1

MIL-C-28950A Valid Notice 1

MIL-P-28958

MIL-J-28978A

MII-M-28985

MIL-C-29106B

MIL-C-29107C

MIL-C-29109B

MIL-T-29112C

MIL-S-29130A

MIL-S-29149C

MIIL-C-29366B

MIL-J-29370

MIL-C-29380D

MIL-V-29389

MII-B-29407A

MIL-S-2915A

MIL-C-29424A

MIL-S-29428A
Trousers, Men's: Service, Summer and Winter (Officer's). FSC 8405

Trousers Men's: Dress (Officer's). FSC 8405

Vest, Man's: Dress White (Officer's). FSC 8405

Coat Man's: Dress Blue (Officer's). FSC 8405

Parka and Trousers, Wet Weather: Lightweight. FSC 8405

Jacket, Man's: Evening Dress (Staff, Noncommissioned

Officer's). FSC 8405

Maintenance Kit: Wet Weather Clothing; Parka and Trousers.

FSC 8405

Coat, Man's, Wool, Winter. FSC 8405

Coat, Man's, Polyester/Wool. FSC 8405

Coveralls, Anti-Exposure. FSC 8415

Trunks Swimmers. FSC 8415

Shorts, Men's, Polyester/Cotton. FSC 8405

Shirt, Man's, Polyester and Wool, Long Sleeve. FSC 8405

Cap, Utility: Camouflage. FSC 8405

Jacket, Man's: Lightweight. FSC 8405

Coat, All-Weather, Men's. FSC 8405

Vest, Man's: Scarlet, Dress (General Officer's). FSC 8405

Belt, All Weather, Coat, Men's. FSC 8405

Sweater; Service Wool. FSC 8405

Coat, Man's: Polyester/Wool, Gabardine. Green (with Belt).

FSC 8405

Scarf: Headover, Wool. FSC 8440 
MIL-C-29433

MIL-J-29451A

MIL-T-29542A

MIL-C-29632

MII-J-29634

MII-C-31002

MIL-C-31006 INT AMD 1

MIL-C-38182C

MIL-P-38184C Valid Notice

MIL-R-38213B Valid Notice 1

MIL-T-41828H

MIL-C-41833E

MIL-T-41834G

MIL-B-43172E

MIL-L-0043335F INT AMD 1

MIL-S-43355B Valid Notice 1

MIL-H-43371B

MIL-C-43415C

MIL-C-43419E

MIIL-C-43455J

MIL-T-43497D (1)

MIL-L-43498D

MIL-R-43518C

MIL-L-43536F

MII-O-4357C

8405

MIL-P-0043700D INT AMD 1 Poncho, Wet Weather. FSC 8405

MII-C-43724C Valid Notice 1 Cap - Hot Weather Olive Green 507. FSC 8415

MIL-P-43907D

MIL-J-43924E

MIIL-S-43929B Valid Notice 1

MIL-T-43932C Valid Notice 1

MIL-M-43946A Valid Notice 1

MIL-T-43957D

MIL-S-43959A

MIL-S-43960 Valid Notice 1

MII-B-43965A Valid Notice 1

MIL-J-43967C

MIL-C-44030B

MIL-S-44039C

MIL-S-44041C

MII-T-44047E

MII-P-44087B INT AMD 1

MIL-L-44089A

MII-T-44094B INT AMD 1

MIL-H-44105B

MIL-C-44211A (1)
Caps, Garrison: Men's. FSC 8405

Jacket, Men's: Intermediate Weight, Polyester/Wool. FSC 8405

Trousers, Men's: Polyester/Wool. FSC 8405

Coat, Man's, Polyester/Wool, Serge, Fusible (Coast Guard).

FSC 8405

Jersey, Flight Deck Crewman's (Flame Retardant). FSC 8415

Cap, Garrison, Man's (Coast Guards). FSC 8405

Coat, Man's. FSC 8405

Cover, Service Cap, Man's, Water Repellant. FSC 8405

Parka, Extreme Cold Weather CWU-8/P. FSC 8415

Raincoat, Man's, Lightweight, Blue. FSC 8405

Trousers, Men's, Polyester/Wool. FSC 8405

Coverall, Mechanic's, Cold Weather. FSC 8415

Trousers, Men's, Polyester and Cotton. FSC 8415

Beret, Man's, Wool. FSC 8405

Liner, Wet Weather Poncho. FSC 8405

Strap Chin; and Suspension Assembly, Chinstrap. FSC 8470

Hat, Sun, Reversible. FSC 8415

Cap, Service, Military Police, White. FSC 8405

Cap, Garrison, Men's Polyester/Wool, Army Green 344. FSC 8405

Coat, Cold Weather, Field. FSC 8415

Trousers, Cold Weather, Field, Nylon and Cotton. FSC 8415

Liner, Cold Weather Trousers, Field. FSC 8415

Raincoats, Men's, Quarpel. FSC 8405

Liner, Cold Weather Coat. FSC 8415 Overcoat, Man's, Army Green 44, with Removable Liner. FSC

Parka and Trousers, Wet Weather. FSC 8405

Jackets, Cold Weather, (High Temperature Resistant). FSC 8415

Shirt, Utility, (Durable Press). FSC 8405

Trousers, Utility, (Durable Press). FSC 8405

Maintenance Kit: Wet Weather Clothing. FSC 8405

Trousers, Men's, Dress, Wool and Polyester/Wool. FSC 8405

Sweat Shirt: Zipper Front. FSC 8415

Sweat Pants: Leg Zipper. FSC 8415

Bag, Wet Weather Clothing: (Parka and Trousers). FSC 8465

Jersey, Reversible. FSC 8415

Coat, All-Weather, Man's, Black, with Removable Liner. FSC 8405

Shirt, Man's, Long Sleeve, Polyester/Cotton, Army Green 415, Durable Press. FSC 8405

Shirt, Man's, Short Sleeve, Polyester/Cotton, Army Green 415, Durable Press. FSC 8405

Trousers, Camouflage Pattern, Combat. FSC 8415

Parka, Night Camouflage, Desert. FSC 8415

Liner, Night Camouflage Parka: Desert. FSC 8415

Trousers, Night Camouflage, Desert. FSC 8415

Hats, Sun, Hot Weather. FSC 8415

Coats, Men's, Tropical and Serge, Polyester/Wool, Army Green

344, Fusible. FSC 8405 
MIL-S-44212A

MII-T-44214A

MII-S-44215A

MIL-S-44290

MIL-T-44291

A-A-50358B

A-A-50366

A-A-50367A

A-A-50369

A-A-50526B

A-A-50527

A-A-50528A

A-A-52112B

A-A $-52115 B$

A-A-55085

A-A-55086

A-A-55091

A-A-55095

A-A-55108

A-A-55110A

A-A-55178

A-A-55180

A-A-55184

A-A-55185

A-A-55186

A-A-55219

A-A-55222

A-A-55229

A-A-55239

A-A-55294

MIL-C-82114A

MIL-T-82139A

MIL-C-82145A Valid Notice 1

MIL-C-82149A Valid Notice 1

MIL-S-82155B

MIL-C-82156B

MIL-H-82157A

ML-T-82161A Valid Notice 1

MILL-T-82163B

MIL-C-82168A Valid Notice 1

MIL-C-82172B (1)

MIL-J-82193B

MII-O-82250D

MIL-T-82251E

MIL-J-82293D

MIL-J-83472A Valid Notice 1

MIL-C-87000B (1)
Sweatshirt, Hooded, Physical Fitness Uniform (PFU). FSC 8415

T-Shirt, Physical Fitness Uniform (PFU). FSC 8415

Sweatpants, Physical Fitness Uniform (PFU). FSC 8415

Smock, Man's: Hospital Duty Uniform (HDU). FSC 8405

Trousers, Man's: Hospital Duty Uniform (HDU). FSC 8405

Coveralls, Disposable, General Purpose. FSC 8415

Sweater, Man's Modacrylic/Wool. FSC 8405

Coat, Man's, All Weather, with Removable Liner. FSC 8405

Cap, Knit (Watch). FSC 8405

Hat, Service: with Chin Strap. FSC 8405

Trunks, General Purpose. FSC 8415

Coat, Shooter's Green. FSC 8415

Shirts, Man's. FSC 8405

Sweater, Man's, Olive Drab. FSC 8405

Trousers, Men's, Polyester/Cotton. FSC 8405

Trousers, Men's, Undress, Polyester/Cotton (CG). FSC 8405

Shirt, Man's, Polyester and Wool, Long Sleeve. FSC 8405

Coveralls, Utility. FSC 8405

Hat, Service (White). FSC 8405

Coat, All-Weather, Man's, W/Removable Liner. FSC 8405

Coveralls, Men's, Cotton, Sateen. FSC 8405

Cap, Utility, Cotton, Sateen, Green. FSC 8405

Beret, Man's, Wool. FSC 8405

Trousers, Men's (White). FSC 8405

Poncho, Wet Weather, Heavy Duty. FSC 8405

Trousers, Men's (Enlisted, White). FSC 8405

Belt, Man's Coat. FSC 8405

Raincoat, Man's. FSC 8405

Sweater, Service Wool. FSC 8405

Cap, Camouflage Pattern. FSC 8415

Coat Front: for Coats, Musicians. FSC 8315

Tabard: USMC Band, Embroidered. FSC 8345

Coat, Man's: Special Full Dress Scarlet, U.S. Marine Band, Musicians. FSC 8405

Coat, Man's: Full Dress, Scarlet, U.S. Marine Band, Musician.

FSC 8405

Stripe, Trousers, Dress. FSC 8455

Coat, Man's; Scarlet, Drum and Bugle Corps, Musician. FSC 8405

Hat, Rain: Man's, Cotton, Rubber Coated; Olive Green 107. FSC 8405

Trousers, Men's: Evening Dress (Officer's). FSC 8405

Trousers, Men's: Dress, White and Special Mess, Black. FSC 8405

Coat, Man's, Full Dress, Summer and winter, Scarlet, U.S.

Marine Band, Drum Major. FSC 8405

Coat, Man's: Full Dress, U.S. Marine Band Officer's. FSC 8405

Jacket, Man's: Evening Dress (Officer's). FSC 8405

Overcoat, Man's (Officer's Type). FSC 8405

Trunks, General Purpose. FSC 8415

Jacket, Utility, Man's, Blue. FSC 8405

Jacket, Cold Weather, Security Police CWU-46/P. FSC 8415

Coveralls, Men's. FSC 8405 
MIIL-C-87026A

MIL-J-87035C

MIL-J-87037D

MII-T-87038D

MIL-H-87041B

MIL-S-87046A

MIL-T-87047A

MIL-S-87060B

MIL-T-87062B

MIL-T-87067C

MIL-C-87093B

MIIL-P-97098

MIL-T-87099

MIL-C-87110A

MIL-C-87165 Valid Notice 1 MIL-S-87214B

MIIL-J-87250

Outerwear, Women's

MIL-STD-656C

MII-STD-657A

MIIL-STD-902A

MIL-STD-984 Chg Notice 1

MIL-STD-1608C Notice 1

MII-STD-1609C

A-A-1626

A-A-1782

A-A-1783

A-A-1784

A-A-1785

A-A-1786

MIL-C-15065J

MIL-H-15505K

MIL-C-15507L

MIIL-C-15881C

MIL-S-19665B

MIL-H-19793C

MIL-S-202474A

MIIL-O-21086B (1)

MIL-H-24900A

MIL-R-24919A

MIL-C-24921A

MIL-S-24923

MIL-O-24926A

MIL-S-24948A

MIIL-J-24949

MIL-C-28922 (2)
Coat, Man's, Polyester/Wool, Serge (Coat Guard). FSC 8405

Jumper, Man's (Blue, Dress). FSC 8405

Jumper, Man's, White. FSC 8405

Trousers, Men's (Blue, Enlisted). FSC 8405

Hat, Service (White). FSC 8405

Shirt, Utility, Man's, Polyester/Cotton (CG). FSC 8405

Trousers, Men's Polyester/Wool Serge (CG). FSC 8405

Shirts, Utility, Men's Chambray. FSC 8405

Trousers, Utility, Men's Denim. FSC 8405

Trousers, Men's, (Enlisted, White). FSC 8405

Coveralls, Flame Resistant (Aramid). FSC 84115

Parka, Wet Weather. FSC 8405

Trousers, Wet Weather. FSC 8405

Coat, All-Weather: Man's, Blue, with Removable Liner. FSC

8405

Collar, Jacket, Detachable CWU 63/P. FSC 8315

Shirts, Man's Short and Long Sleeves Polyester/Cotton (Durable

Press) and Long Sleeves, Polyester/Wool. FSC 8405

Jacket, Man's; Lightweight with Removable Liner. FSC 8405

Provisions for Evaluating Quality of Slacks, Women's. FSC 8410

Provisions for Evaluating Quality of Service Caps. FSC 8405

Provisions for Evaluating Quality of Caps, Garrison, Women's.

FSC 8410

Size Labeling for Women's Uniform Clothing, Provisions for.

FSC 8410

Provisions for Evaluating Quality of Coats, Women's, Dress.

FSC 8410

Provisions for Evaluating Quality of Women's Skirts. FSC 8410

Shirt, Man's and Women's (Long or Short Sleeve). FSC 8405

Cap, Civilian, Uniform. FSC 8405

Shirt, Man's (and Woman's; Long Sleeve). FSC 8415

Trousers, Man's (and Woman's - Summer Weight). FSC 8405

Trousers, Man's and Woman's (Winter Weight). FSC 8405

Shirt, Man's (and Woman's; Short Sleeve). FSC 8415

Coat Fronts. FSC 8315

Hat, Service, Woman's. FSC 8410

Cap, Garrison, Woman's (Navy). FSC 8410

Coat, Woman's: Cotton, Sateen; Green; (Utility). FSC 8410

Shirt, Woman's: Cotton, Sateen, Green (Utility). FSC 8410

Havelock, Plastic. FSC 8410

Slacks, Women's: Cotton, Sateen, Green (Utility). FSC 8410

Overcoat, Women's: Wool, Serge, Green. FSC 8410

Hat, Combination, Woman's (Coast Guard). FSC 8410

Raincoat, Women's, w/Removable Liner(CG). FSC 8410

Coat, All-Weather, Woman's, with Removable Liner. FSC 8410

Shirt, Utility, Woman's, Polyester/Cotton (CG). FSC 8410

Overcoat, Woman's, Enlisted. FSC 8410

Slacks, Women's (with Side Pockets). FSC 8410

Jumper, Woman's, White. FSC 8410

Coat, Woman's: Summer, Green and White. FSC 8410 


MIL-U-28946A Valid Notice 1
MIL-S-29122D
MIL-C-29123A
MIL-C-29124D
MIL-S-29138A (1)
MIL-S-29368C
MIL-T-29375A
MIL-S-29376A
MIL-S-29377A
MIL-C-29381C
MIL-H-29382
MIL-V-29384 Valid Notice 1
MLL-C-29386A
MIL-S-29388B (1)
MIL-C-29391A

MIL-C-29393 Valid Notice 1

MIL-S-29394B

MIL-S-29395A

MIL-J-29396 Valid Notice 1

MIL-J-29397 Valid Notice 1

MIL-B-29408A

MII-C-29427A (1)

MIL-S-29429A

MIL-C-29431

MIL-S-29432A

MIL-C-29453A

MII-C-29454

MII-C-29628

MIL-S-29629

MIL-S-29630

MIL-S-29631

MIL-S-29633A

MIL-D-37031

MIL-S-40035C (2)

MIL-S-41825F

MIL-H-43162F

MIL-S-43505D

MIL-C-43972D

MIL-S-44090C

MIL-S-44092B (1)

MIL-S-44093B

MIIL-S-44102B

MII-C-44107C

MIL-S-44110B
Uniform, Women's: White; Dress (Officer's). FSC 8410

Skirt, Woman's, Blue, Dress. FSC 8410

Coat, Women's, Summer (Navy). FSC 8410

Coat, Woman's, Blue, Dress. FSC 8410

Sweater, Woman's, Acrylic. FSC 8410

Shirts, Women's: Long and Short Sleeves. FSC 8410

Tunic: Woman's Maternity. FSC 8410

Skirt: Woman's Maternity. FSC 8410

Slacks: Women's Maternity. FSC 8410

Coat, All-Weather, Women's. FSC 8410

Hood, Woman's: All-Weather Coat, Dress. FSC 8410

Vest, Women's: Scarlet, Dress (General Officer's). FSC 8410

Cap, Dress: Women's. FSC 8410

Shirts, Women's: Maternity, Long and Short Sleeves. FSC 8410

Coat, Women's: Wool Gabardine: Dress Blue Ceremonial. FSC 8410

Coat, Women's: Full Dress, Scarlet, U.S. Marine Band, Musician's. FSC 8410

Skirts, Women's: Evening Dress, U.S. Marine Band

(Musician's). FSC 8410

Slacks, Women's; Musician's. FSC 8410

Jacket, Women's; Special Full Dress Scarlet, U.S. Marine Band, Musician. FSC 8410

Jacket, Woman's, Full Dress Scarlet, U.S. Marine Band, Musician. FSC 8410

Belt, All Weather Coat, Women's. FSC 8410

Coats, Women's. FSC 8410

Skirts, Women's. FSC 8410

Caps, Garrison: Women's. FSC 8410

Slacks, Women's. FSC 8410

Coat, Woman's: Wool/Polyester; Gabardine, Blue. FSC 8410

Caps, Service: Women's, Polyester/Wool, Wool. FSC 8410

Coat, Woman's, Blue, Dress. FSC 8410

Skirt, Woman's (with Welt Pockets). FSC 8410

Slacks, Women's. FSC 8410

Skirt, Woman's, Blue, Dress. FSC 8410

Shirt, Woman's, Dress, Short and Long Sleeve (Coast Guard).

FSC 8410

Dresses, Woman's, Cotton-Polyester, Static Resistant, Pleated

Front. FSC 8410

Shirt, Woman's (Exercise). FSC 8415

Slacks, Women's. FSC 8410

Hat, Service, Woman's, Wool or Polyester and Wool. FSC 8410

Shirt, Woman's, Polyester/Cotton. FSC 8410

Coat, All-Weather, Woman's, Black with Removable Liner. FSC 8410

Shirt, Woman's, Short Sleeve, Polyester/Cotton, Army Green 415, Durable Press. FSC 8410

Slacks, Women's: Classic Design, Polyester/Wool. FSC 8410

Shirt, Woman's, Long Sleeve, Polyester/Cotton, Army Green

415, Durable Press. FSC 8410

Skirt, Woman's, Classic Design, Polyester/Wool. FSC 8410

Cover, Ground Troops-Parachutists Helmet. FSC 8415

Slacks, Maternity, Utility Work Uniform. FSC 8410 
MIL-C-44111B

MIL-C-44130C

MIL-T-44293A

MIIL-C-44379 (1)

A-A-50011 Valid Notice 1

A-A-50072A

A-A-50365A

A-A-50368A

A-A-50527

A-A-55111A

A-A-55122

A-A-55189

A-A-55210

A-A-55212

A-A -55218

A-A-55221

A-A-55230

MII-C-82104 (1)

MIL-C-82114A

MIL-J-82122B

MIL-C-82125A

MIL-S-82126B Valid Notice 1

MIIL-H-82142 (1)

MIL-R-82190A (1)

MII-H-83012C

MIL-S-83234C

MIL-B-83268B Valid Notice 1

MIL-H-83269A Valid Notice 1

MIL-C-83422

MIL-S-83482B

MIL-S-87005D

MIL-S-87006A

MIL-S-87012D

MIL-S-87013D

MIL-C-87014B

MIL-S-87053C

MIL-S-87054C

MIIL-S-87055B (1)

MIIL-S-87056A

MIL-J-87056A

MIL-S-87061C

MIL-S-87063B

MIIL-S-87073

MIL-C-87076A

MIL-S-87091C

MIL-C-87160A

MIL-C-87215

MIL-S-87225A
Coat, Maternity, Utility Work Uniform. FSC 8410

Cap, Garrison, Women's, Polyester/Wool, AG-344. FSC 8410

Tunic, Woman's, Hospital Duty Uniform (HDU). FSC 8410

Coats, Woman's, Classic Design, Polyester/Wool, AG-344. FSC

8410

Uniform, Woman's: Tunic and Slacks, White, Food Handlers.

FSC 8410

Hat, Service, Woman's, Drill Instructor. FSC 8410

Sweater, Woman's, Acrylic. FSC 8410

Coat, Woman's, All-Weather, with Removable Liner. FSC 8410

Trunks, General Purpose. FSC 8415

Coat, All-Weather, Woman's, w/Removable Liner. FSC 8410

Hood, Rain, Woman's. FSC 8410

Skirt, Maternity. FSC 8410

Clacks, Women's, Undress. FSC 8410

Belt, All-Weather Coat, Women's. FSC 8410

Shirt, Women's, Dress, White (Short Sleeve). FSC 8410

Tunic, Maternity. FSC 8410

Slacks, Woman's Hospital Duty Uniform (HDU). FSC 8410

Cap, Garrison, Woman's; Cotton, Polyester, Dark Blue; (Utility).

FSC 8410

Coat Front: for Coats, Musicians. FSC 8315

Jacket, Woman's: Evening Dress (Officer's). FSC 8410

Cape, Woman's: Evening Dress (Officer's). FSC 8410

Skirts, Women's: Evening Dress. FSC 8410

Hood, Rain, Woman's: Nylon, Rubber Coated; Green. FSC

8410

Raincoat, Woman's: Nylon, Rubber Coated; Green. FSC 8410

Hat, Service, Woman's (Frame and Removable Cover). FSC

8410

Skirts, Women's, Blue. FSC 8410

Beret, Woman's. FSC 8410

Hood, Rain, Woman's. FSC 8410

Cap, Woman's, Hot Weather. FSC 8410

Slacks, Women's. FSC 8410

Skirt, Woman's. Dress (CG). FSC 8410

Shirt, Woman's. Dress, (Coast Guard). FSC 8410

Slacks, Women', Dress (CG). FSC 8410

Slacks, Women's, Undress. FSC 8410

Cap, Garrison, Woman's (Coast Guard). FSC 8410

Skirt, Woman's, Belted. FSC 8410

Slacks, Women's, Belted. FSC 8410

Shirt, Woman's, Dress, White (Short Sleeve). FSC 8410

Shirt, Woman's Dress Blue (Long Sleeve). FSC 8410

Jacket, Utility, Woman's. FSC 8410

Shirts, Utility, Women's Chambray. FSC 8410

Slacks, Utility, Women's, Denim. FSC 8410

Shirt, Woman's, Working, Khaki (Long Sleeve). FSC 8410

Cloth, Coated, Aramid, Aluminized. FSC 8305

Shirt, Woman's, Open Notch Collar. FSC 8410

Coat, Woman's, (Pant Suit). FSC 8410

Cap, Garrison, Woman's, Sir Force. FSC 8410

Shirts, Women's: Short and Long Sleeves, Polyester/Cotton

(Durable Press) and Long Sleeves, Polyester/Wool. FSC 8410 
Food Handler's/Processor's

A-A-91B

DOD-A-616G Valid Notice 1

BBB-F-695 (1)

A-A-719

MIL-P-1601E

MIL-S-1820G

MIL-D-3018D Reinst Notice 2

MIL-C-15096H

MIL-C-19479B

MII-C-29136

MIL-L-44042

A-A-50380

A-A-55067

Special Pockets Garments

MiI-V-44323A (1)

MIIL-V-44362 (1)

A-A-55227

A-A-55240

MIL-V-81523A Valid Notice 2 MIL-V-83271B

Surgical Gown/Glove/Mask

DOD-C-48E

A-A-30119A

A-A-30153

A-A-30156

A-A-30188

MIL-M-36168

MIL-G-36565A Valid Notice 1

MIL-S-36573B (2)

MIL-F-36972 Valid Notice 1

MIL-F-36978 (1)

MIL-T-37030 Valid Notice 1

MIL-S-37039 Valid Notice 1

MIL-T-37046 Valid Notice 1

MIL-T-37064 Valid Notice 1

MIL-T-37069 Valid Notice 1
Apron, Food Handlers. FSC 8415

Aprons, Food Handlers'. FSC 8415

Frock, Man's (Butcher's, White). FSC 8415

Food Handler's Paper Caps. FSC 8415

Protectors, Arm, Gasoline Field Range Outfit. FSC 7360

Smock, Food Inspector's. FSC 8415

Dress, Food Handler's, Woman's. FSC 8415

Coat, Food Handler's (Steward). FSC 8415

Coat, Food Handler's: Cotton and Polyester Twill; White; with Pocket. FSC 8405

Cap, Food Handler's. FSC 8415

Liner, Food Inspector's Smock. FSC 8415

Coat, Food Handler's (Steward). FSC 8415

Smock, Food Inspector's, FSC 8415

Vest, Tactical Load Bearing. FSC 8415

Vest, Grenade, Carrier (for 40-mm Grenades). FSC 8415

Pocket, Ammunition Magazine, Enlisted Men's, M-1923. FSC 8465

Apron, Construction Worker's. FSC 8415

Vest, Survival Equipment, Type SV-2A. FSC 8415

Vest, Survival Mesh Set, SRU-21/P. FSC 8415

Cap, Operating, Surgical, Green. FSC 6532

Hood, Operating, Surgical. FSC 6532

Mask, Surgical. FSC 6515

Cap, Operating, Surgical (Woman's). FSC 6532

Mask, Surgical (Sub-Micron). FSC 6515

Mask, Surgical, Nonwoven Fabric, Green, Disposable. FSC 6510

Gowns, Operating, Surgical, Cotton, Vest-Type, Green. FSC 6532

Smock, Dental Operating. FSC 6532

Gown, Operating, Surgical. FSC 6532

Footwear Covers, Disposable, Conductive Plastic Film. FSC 8430.

Trousers, Operating, Surgical Men's, Cotton-Polyester, Static Resistant. FSC 6532

Shirts, Operating, Surgical, Man's Cotton-Polyester Static Resistant. FSC 6532

Trousers, Operating, Surgical, Women's, Cotton-Polyester, Static Resistant. FSC 6532

Tunics, Operating, Surgical, Woman's Cotton-Polyester, Static Resistant, Long Sleeves. FSC 6532

Tunics, Operating, Surgical, Woman's Cotton-Polyester, Static Resistant, Short Sleeves. FSC 6532 
MIL-S-37129 Valid Notice 1

MIL-S-37130 Valid Notice 1

MIL-T-37131 Valid Notice 1

A-A-51070A

A-A-51264B

A-A-51301A

A-A-51343

A-A-51361A

A-A-51373A

A-A-0053134

A-A-53443

A-A-54252

A-A-54372A

A-A-54407

A-A-54433

A-A-54435

A-A-54553

A-A-54791

A-A-54807

A-A-54870
Shirts, Operating, Surgical, Man's, Cotton, Sleeveless, Style A. FSC 6532

Shirts, Operating, Surgical, Man's, Cotton, Quarter Length

Sleeves, Style B. FSC 6532

Trousers, Operating, Surgical, Man's Cotton, Green. FSC 6532 Mask, Surgical. FSC 6515

Surgical Pack, Gown and Towel. FSC 6532

Footwear Covers, Operating Room (Disposable). FSC 6532

Surgical Pack, Lower Extremity. FSC 6532

Surgical Pack, Gown and Towel. FSC 6532

Gown Operating, Surgical. FSC 6532

Gloves, Surgeons' (Powder-Free, Sterile, Disposable). FSC 6515

Gown, Operating, Surgical. FSC 6532

Surgical Pack, Disposable (Arthroscopic). FSC 6530

Mask, Surgical (Pleated). FSC 6532

Gown, Operating, Surgical. FSC 6532

Cap, Operating, Surgical. FSC 6532

Mask, Surgical (Non-Woven Fabric, Pouch). FSC 6515

Mask, Surgical. FSC 6515

Gloves, Surgeons', Latex Rubber, Pre-Powdered, Disposable, Sterile. FSC 6515

Gloves, Surgeons', Brown-Milled Rubber, Pre-Powdered, TalcFree, Disposable, Sterile. FSC 6515

Caps, Operating, Surgical (Woman's). FSC 6532

Nonsurgical Medical \& Veterinary

MIL-S-2021E Reinst Notice 2 Suit, Convalescent (Jack and Trousers). FSC 6532

MIL-C-37186B

MIL-S-37442

Coats, Medical Attendant's, Man's, White, Cotton-Polyester,

Durable Press. FSC 6532

MII-S-0037951

Smocks, Physician's, Man's, White, Cotton-Polyester, Durable

Press. FSC 6532

MIL-D-43732F

Smock, Medical Assistant's, Man's, White, Cotton-Polyester, Durable Press. FSC 6532

A-A-53562

Dress, Woman's, Hospital Duty Uniform (HDU). FSC 8410

Glove, Patient Examining and Treatment (Plastic, Large Size,

Sterile). FSC 6515

A-A-54373

A-A- 54480

Robe, Dressing (Striped Seersucker). FSC 6532

A-A-54916

Mask, Face, Asceptic. FSC 6532

Gown, Hospital Patient. FSC 6532

Underwear \& Nightwear, Men's
A-A-153
MIL-D-2525D
MIL-U-2526D
MIL-D-40099H
MIL-U-43262D
MII-S-43357E
MIL-D-43357E
MIL-U-44096A (1)
MIL-U-44164A

Drawers, Men's. FSC 8420

Drawers, Men's: Cotton, Ankle Length. FSC 8420

Undershirts, Man's: Cotton, Full Length Sleeves. FSC 8420

Drawers, Men's Boxer Style. FSC 8415

Undershirts, Cold Weather, Men's. FSC 8415

Shirt, Sleeping, Heat Retentive and Moisture Resistant,

Nylon/Acetate, Tricot Knit. FSC 8415

Drawers, Men's, Brief Type. FSC 8420

Undershirt, Man's (Quarter-Sleeve). FSC 8420

Undershirt, Cold Weather, Polypropylene. FSC 8415 
A-A-50003B

A-A-50013D

A-A-50353
Drawers, Men's (Brief-Type). FSC 8420

Undershirt, Man's (Quarter-Sleeve). FSC 8420

Undershirt, Man's (Polyester/Cotton) Quarter Sleeve (Use A-A50013). FSC 8420

Underwear \& Nightwear, Women's

MS35839

Underwear and Nightwear, Women's FSC Class 8425. FSC 8425.

Hosiery, Handwear \& Clothing Accessories, Men's

MIL-S-48L

A-A-114

MIL-S-405H

MIL-L-714G

MIL-STD-1612B

MIL-STD-1613B

A-A-1624

A-A-1787

MIL-G-3866G

MIL-S-5365F Valid Notice 1

MIL-S-10926G

MIL-S-11922E Valid Notice 1

MIL-S-14210G

MIL-M-16149G

MIL-C-19677C

MIL-C-19688B

MIL-G-21893C

MIL-P-22295C Valid Notice 1

MIL-G-24909A

A-A-30052B

MS35807

MIL-N-41804E

MIL-G-41817E

MIL-B-43515A Valid Notice 1

MIL-N-43741B

MIL-S-43823A Valid Notice 1

MIL-G-44108A

A-A-50015B

A-A-50016A

A-A-50021A

A-A-50356B

A-A-50386

A-A-52055

A-A-52203

A-A-55079
Socks, Men's, Cushion Sole, Stretch Type. FSC 8440

Socks, Cotton. FSC 8440

Socks, Men's, Winter (Wool and Cotton). FSC 8440

Leggings, Men's. FSC 8440

Provisions for Evaluating Quality of Gloves, Cloth, Dress. FSC 8440

Provisions for Evaluating Quality of Gloves, Leather, Dress.

FSC 84GP

Necktie (Striped). FSC 8440

Necktie. FSC 8440

Gloves, Cloth, Cotton, Knitted, Lightweight. FSC 8415

Scarf, Neckwear, Sage Green, Flying, Tubular, N-18 (Scarf,

Sage Green, Flying, tubular, Type N-18). FSC 8440

Suspenders, Trousers, M-1950. FSC 8440

Scarf, Branch of Service, BIB Type. FSC 8455

Socks, Men's, Nylon and Cotton, Knee Length, Stretch Type.

FSC 8440

Mitten, Welders. FSC 8415

Clasp, Necktie: Metal; Gold Colored. FSC 8455

Cummerbund, Man's: Black. FSC 8440

Gloves, Cloth, Nylon, Knitted (Dress, Men's). FSC 8440

Protector Trousers, Pistol Holster, FSC 8465

Gloves, Men's and Women's. FSC 8440

Socks, Men's. FSC 8440

Hosiery, Handwear, and Clothing Accessories: Men's FSC Class 8440. FSC 8440

Neckties, Men's, Four-in-Hand. FSC 8440.

Gloves, Men's, Cloth, Dress, White. FSC 8440

Belt, Man's, Waist, Blue 334 (Army Band Uniform). FSC 8405

Handkerchief, Ham's, Cotton, Knitted. FSC 8440

Socks, Men's, Nylon, Cushion Sole, Stretch Type, OG 106.

FSC 8440

Gloves, Combat Vehicle Crewman's, Summer. FSC 8415

Socks, Ribbed Knit, Stretch Type. FSC 8440

Gloves, Men's: Cloth, Leather Palm, Knitted Wristlet, Size

Medium. FSC 8415

Gloves, Men's Cloth, Leather Palm with Gauntlet. FSC 8415

Handkerchief, Men's or Women's. FSC 8440

Gloves, Men's and Women's. FSC 8440

Gloves, Men's and Women's, Leather, Light Duty. FSC 8415

Suspenders, Trousers (Flying Suit). FSC 8440

Socks: Men's, Cushion Sole, Stretch Type. FSC 8440 
A-A-55083

A-A-55107

A-A-55199

A-A-55203

A-A-55226

A-A-55236

A-A-55246A

MIL-C-82167 Valid Notice 1

MIL-N-87042C
Scarf, Neckwear, Sage Green, Flying, Tubular N-1B (Scarf, Sage Green, Flying Tubular, Type N-18). FSC 8440

Socks: Men's, Nylon, Cushion Sole, Stretch Type. FSC 8440

Necktie. FSC 8440

Suspenders, Trousers (Flying Suit). FSC 8440

Scarf, Neckwear, Wool. FSC 8440

Gloves, Men's, Cloth, Dress, White. FSC 8440

Gaiter, Neck. FSC 8440

Gloves, Leather: Gauntlet; Drummer's. FSC 8440

Neckerchief (Acetate Black). FSC 8440

Hosiery, Handwear \& Clothing Accessories, Women's

MIL-G-1007H Gloves, Women's. FSC 8445

MIL-STD-1611A Valid Notice 1Provisions for Evaluating Quality of Hoods and Havelocks,

A-A-1787

Woman's. FSC 8410

MII-S-10679E Valid Notice 1 Scarf, Neckwear: Woman's. FSC 8445

MIL-S-17868B (1)

Scarf, Neckwear: Wool, Women's. FSC 8445

MIL-N-19857C Reinst Notice 2Neckties: Women's, Polyester/Wool. FSC 8445

MIL-G-24909A

MIL-N-29113B

MIL-A-29131B

MIL-N-29387A

MII-S-43317C

Gloves, Men's and Women's. FSC 8440

Necktie Woman's (Bow, Black). FSC 8445

Anklets, Woman's, Acrylic and Nylon, Ribbed, Stretch Type.

FSC 8445

Necktie, Women's: General Officer. FSC 8445

MIIL-G-43958 Valid Notice 1

MIL-H-43981D

Scarf, Neckwear, Woman's. Acrylic. FSC 8445

Gloves, Cloth, Black, Lined, Girl's Jr. R.O.T.C.. FSC 8445

Handbag, Women's, Synthetic, Black (Use A-A-55113). FSC 8445

MIL-N-44106B

Necktab, Woman's Shirt. FSC 8445

A-A-50386

Gloves, Men's and Women's. FSC 8440

A-A-52055

A-A-55073

Gloves, Men's and Women's, Leather, Light Duty. FSC 8415

A-A-55113

A-A-55225

A-A-55226

MIL-C-82111A

MIL-O-82112A

Necktie, Woman's (Coast Guard). FSC 8445

Handbag, Women's: Synthetic, Black. FSC 8445

Scarf, Neckwear, Women's Acrylic. FSC 8445

Scarf, Neckwear, Wool. FSC 8440

Cover, Purse: Women's (Officer's). FSC 8410

Ornamentation: for Uniform, Woman's, Evening Dress, Officer's.

FSC 8455

MII-C-82121B

Cummerbund, Woman's: Evening and Mess Dress (Officer's). FSC 8445.

MIL-G-83150A Valid Notice 1 Gloves, Cloth, Nylon Knitted (Women's, Dress). FSC 8445

MII-N-87007B

Necktie, Woman's (CG). FSC 8445

Children's \& Infant's Apparel \& Accessories

A-A-54036

Undershirt, Infant's. FSC 6532

Luggage

KK-S-151C

A-A-584B Valid Notice 1

KK-B-650A INT AMD 2

MII-B-829M
Satchels, Physician's. FSC 6532

Case, General Utility (Artificial Leather). FSC 8460

Briefcase (Leather). FSC 8460

Bag, Duffel. FSC 8465 


A-A-1519A
MIL-B-2378H
A-A-2523B
A-A-2724
MIL-T-10798L
MIL-T-16381B
MS35860
MIL-S-37180 Valid Notice 1
MIL-K-41835D
A-A-50083
A-A-55062A
A-A-55179
A-A-55192
A-A-55205
MIL-C-81808
MIL-K-83782A Valid Notice 1
MUL-S-83791A
MIL-B-87018A

Individual Equipment

MIL-F-411D

MIL-B-833G

MIL-C-1002J

A-A-1040A

MIL-B-1107G

NAF 1197 REV 2

MIL-B-1462F

MIL-P-1474J

MIL-C-1476G

MIL-S-1478F

MIIL-B-1718H

MIL-S-1812C

MIL-P-1814E Valid Notice 1

MIL-C-1933G

MIL-B-2883D

MIL-C-3880E

AN8018 Rev A Valid Notice 1

AN8019 Rev 1 Valid Notice 1

MIL-S-10055D

MIL-P-10941D Valid Notice 1

MIL-H-13102D

MIL-B-17693e (1)

MIL-C-17774A Valid Notice 1

MIL-C-17841B (2)

MIL-P-17863C (1)

MLL-C-17864C

MIL-B-18184B Valid Notice 1

MII-S-19206D
Case, Dispatch (Artificial Leather). FSC 8460

Bag, Barracks. FSC 8465

Case, Dispatch, Molded Plastic. FSC 8460

Portfolio, Plastic. FSC 7510

Trunk Locker, Barracks. FSC 8460

Trunk, Locker, Barracks; and Tray. FSC 8460

Luggage FSC Class 8460 . FSC 8460

Satchel, Physician's, Boston Style, Three Compartments. FSC 6532

Kit Bag, Flyer's. FSC 8460

Bag, Plastic, Folded Garment. FSC 8105

Suitcase, Flyers. FSC 8460

Bag, Money. FSC 8460

Case, Map. FSC 8460

Bag, Personal Effects. FSC 8465

Chest, Collapsible. FSC 8460

Kit Bag, Flyer's. FSC 8460

Suitcase, Flyer's Clothing. B-4B. FSC 8460

Bag, Money. FSC 8460

Fasteners, Belt; Clips, End Strap with Hook; and Keepers, Slide. FSC 8465

Belt, Trousers, Cotton Webbing, with Clip. FSC 8440

Case, Field, First Aid Dressing, Leather (Military Police). FSC 8465

Key Chain, Reel (Door Key and Drill Chuck Keys). FSC 5340

Belt, Individual Equipment, M-1936. FSC 8465

Tube Pilot's Relief. FSC 1680

Belt, General Officer's. FSC 8440

Pitons, Mountain. FSC 8465

Creepers, Ice. FSC 8465

Snap Link, Mountain Piton. FSC 8465

Belt, Military Police, 1-3/4 Inch Wide, Man's. FSC 8465

Shelf, Cargo Support, Packboard, Pressed Steel. FSC 8465

Pad, Shoulder, Packboard. FSC 8465

Carrier, Policeman's Club: and Grommet. FSC 8465

Boatswain's Pipe. FSC 8465

Club, Policeman's. FSC 8465

Horn, Flyer's Relief Tube. FSC 4730

Tee and Flyer's Relief Tube. FSC 4730

Strap, Packboard: Quick Release. FSC 8465

Packboard, Plywood. FSC 8465

Holder, Cartridge, Belt, Cal. .38, Leather, Black, 6-Round. FSC 8465

Belts, Coats, Man's: Polyester/Wool. FSC 8405

Cover, Bayonet; Scabbard; Cotton Duck, White (with Leather

Tip). FSC 8465

Carrier, Club, Policeman's: Cotton Webbing; White. FSC 8465

Pocket, Ammunition Magazine: Military Police. FSC 8465

Carrier, Pistol Holster: Cotton Duck, White (MP). FSC 8465

Belt, Coat, Man's, Webbing, Cotton, White. FSC 8440

Sword and Scabbard (Noncommissioned Officers). FSC 8465 
MIL-C-19734C

MIL-C-20006F

MIL-C-20267C Valid Notice 1

MIIL-K-20277H

MIL-S-21042C

MIL-B-21154C

MII-H-21155D

MII-B-21880D

MS22025 Valid Notice 1

MIL-S-28921A

MII-S-28933B

MIL-B-29378 (1)

MIL-C-36828A Valid Notice 1

MIL-S-40022E

MII_S-40046D Valid Notice 1

MIIL-C-4012E (2)

MIL-C-40126F

MII-40131C

MIL-F-40165C Reinst Notice 2

MII-S-43013C Valid Notice 1

MII-C-43103D

MIL-S-43279D

MIL-P-43304C

MIL-S-43306B

MIL-P-43312C

MIL-R-43323E

MIL-S-43489D

MIL-C-43603B (1)

MIL-F-43673 Valid Notice 1

MIL-C-43689C

MIL-L-43720C (1)

MIL-C-43742B

MIIL-P-43756

MIL-M-43757A

MIL-C-43761C

MIL-R-43826C

MIL-S-43828A INT AMD 2

MIL-S-43829B INT AMD 1

MIL-C-43830B INT AMD 1

MIL-C-43831B INT AMD 1

MIL-F-43832C (1)

MIL-F-43833D

MIL-F-43834E

MIL-S-43835E
Carrier: Sword Scabbard. FSC 8465

Clothes Stop. FSC 8465

Carrier, Club, Policeman's: Cotton Webbing, Olive Drab (with

Double Hook). FSC 8465

Knife, Combat: and Sheath. FSC 1095

Sling, Flagstaff: Leather, White; with Brass Socket. FSC 8345

Belts, Military Police: Cotton Webbing; White. FSC 8465

Hardware: for Belt, Military Police. FSC 8465

Belt, Military Police (White). FSC 8465

Bracket, Flyers Relief Tube, Vertical. FSC 1680

Sword and Scabbard: (Officer's) with Case. FSC 8465

Sling, Sword, Shoulder: Nylon Webbing, White. FSC 8465

Belt, Man's: Ceremonial, Officers. FSC 8440

Case, Spectacle, Envelope Type, Soft Fabric Lining. FSC 6540

Shoulder Strap, Side Arm, Military Police, Leather, Black. FSC 8465

Sling, Flagstaff. FSC 8345

Canteen, Water, Insulated, Corrosion-Resisting Steel, without

Cup and Cover. FSC 8465

Cup, Water Canteen (for Insulated Canteen). FSC 8465

Cover, Water Canteen, Insulated, Cotton Duck. FSC 8465

Field Pack, Canvas, Combat, M-1961. FSC 8465

Sling, Universal, Individual Load Carrying. FSC 8465

Canteen, Water, Plastic, with Screw Cap. FSC 8465

Slings, Bag and Carrying: Communications Equipment. FSC 8465

Pack and Harness Assembly, Parachutist's Weapons and Individual Equipment. FSC 8465

Sling, Bag and Case Carrying, ST-33. FSC 8465

Pocket, Ammunition Magazine. FSC 8465

Rifle Butt Pocket and Strap Assembly. FSC 8465

Sling, Bag and Case Carrying: ST-35. FSC 8465

Canteen, Water, Collapsible, 2-Quart Capacity. FSC 8465

Frame, Rucksack, Steel. FSC 8465

Cover, Water Canteen, 2-Quart, Collapsible (with Pile Lining).

FSC 8465

Liner, Field Pack. FSC 8465

Cover, Water Canteen, LC-2. FSC 8465

Packboard, Metal. FSC 8465

Modification Kit, Packboard, Radio Carrying. FSC 8465

Cup, Water Canteen, w/Wire Handle, Corrosion-Resisting Steel.

FSC 8465

Belt, Individual Equipment. FSC 8465

Strap, Webbing, Cargo Tie Down, Lightweight Pack Frame, M1972. FSC 8465

Suspenders, Individual Equipment Belt, LC-1. FSC 8465

Cover, Field Pack, Camouflage, LC-1. FSC 8465

Carrier, Intrenching Tool, Hand, Folding, Lightweight, Plastic, LC-1. FSC 8465

Field Pack, Combat, Nylon, Large, LC-1. FSC 8465

Field Pack, Combat, Nylon, Medium, LC-2. FSC 8465

Frame, Field Pack, (Riveted), and Shelf, Cargo Support

(Lightweight), LC-1. FSC 8465

Straps, Pack Frame and Field Pack, Ground Troops. FSC 8465 
MII-F-43997A

MIL-C-44083A

MII-W-44126A

MII-P-44153A

MII-C-44216A

MII-C-44217A

MIL-C-44218

MII-C-44219

MIL-S-44220A

MII-S-44221A

MIL-A-44264

MIL-B-44306B

MIL-C-44307B

MIL-H-44308A

MIIL-S-44309B

MIL-F-44324A

MIL-C-44347

MIL-C-44348

MIL-C-44349

MII-S-44377

MIL-H-48671

A-A-50098A

A-A-50106A

A-A-50112A

A-A-50116A

A-A-50117A

A-A-50118A

A-A-50119A

A-A-50121A

A-A-50125B

A-A-50127A

A-A-50374

A-A-50748 Valid Notice 1

MIL-C-51278D (1)

A-A-52113

A-A-55058

A-A-55059

A-A-55064

A-A-55070

A-A-55077

A-A-55084

A-A-55092

A-A-55105

A-A-55106

A-A-55114

A-A -55120

A-A -55124

A-A -55173
Field Pack, Training. FSC 8465

Carrier, AN/PRC-68 or AN/PRC-68A, Radio Set. FSC 8465

Water, Drinking, Emergency, Flexibly Packaged. FSC 8960

Pocket, Ammunition Magazine, 9 mm. FSC 8465

Canteen, Water, Collapsible, 5-Quart Capacity. FSC 8465

Cap, Water Canteen, 5-Quart, Collapsible. FSC 8465

Carrier and Canteen/Collapsible, 5-Quart Capacity. FSC 8465

Carrier, Canteen, Collapsible, 5-Quart Capacity. FSC 8465

Sleeping Bag, Cold Weather Aircraft Survival Kit (Vacuum

Packed). FSC 8465

Stand, Canteen Cup. FSC 8465

Adapter Kit, M-1 Cap; for Canteen, Water, Insulated. FSC 8465

Bag, Stuff, Sleeping System. FSC 8465

Cover, Bivy, Extreme and Intermediate Cold Weather Sleeping

Systems. FSC 8465

Hood and Socks, Extreme Cold Weather Sleeping System

(ECWSS). FSC 8465

Sleeping Bags, for Sleeping systems. FSC 8465

Field Pack, Large, with Internal Frame: and Pack, Patrol,

Combat. FSC 8465

Carrier, Water Canteen, Cold Weather, CRS. FSC 8465

Canteen, Water, Cold Weather, CRS. FSC 8465

Cup, Water Canteen, Cold Weather, CRS. FSC 8465

Snowshoe, Trail, Magnesium, Snow and Ice Traversing

Equipment (SITE). FSC 8465

Holster, Hip, M12. FSC 1005

Stopper, Hexagon, Irregular. FSC 8465

Stopper, Wired, Wedged. FSC 8465

Piton, Mountain, Angle. FSC 8465

Axe, Ice. FSC 5110

Crampons, Hinged. FSC 8465

Straps, Crampon. FSC 8465

Anchor, Snow, Wired. FSC 8465

Protector, Crampon. FSC 8465

Descender, Figure-8. FSC 8465

Ascenders, Cam Action. FSC 8465

Bag, Fireman's, Utility. FSC 8460

Holster, Hip, Pistol, Semi-Automatic 9 mm. FSC 1095

Cap, Water Canteen, Field, 1 Quart and 2 Quart Canteens. FSC 8465

Handcuffs and Leg Irons. FSC 8465

Club, Policeman's. FSC 8465

Carrier, Policeman's Club, with Grommet. FSC 8465

Lanyard, Individual Equipment Carrying. FSC 8465

Bag, Wet Weather Clothing: (Parka and Trousers). FSC 8465

Bag, Duffel. FSC 8465

Pocket, Ammunition Magazine. FSC 8465

Bag, Clothing, Waterproof. FSC 8465

Bag, Barracks. FSC 8465

Whistle, Ball, Plastic. FSC 8465

Bag, Laundry. FSC 8465

Lanyard, Individual Equipment Carrying. FSC 8465

Creepers, Ice. FSC 846

Belt, Military Police, 1-3/4 Inch Wide, Man's. FSC 8465 
A-A-55176

A-A-55177

A-A -55182

A-A-55191

A-A-55193

A-A-55194

A-A-55197

A-A -55207

A-A-55231

A-A-55238

A-A-55245

MIL-B-63992A Valid Notice 1

MIL-C-82141A

MIL-B-83475 Valid Notice 1

MIIL-B-83665B
Bag, Laundry, Nylon. FSC 8465

Suspenders, Individual Equipment Belt, LC-2. FSC 8465

Cover, Personnel Identification Tag. FSC 8465

Shoulder Strap, Side Arm, Military Police, Leather, Black. FSC 8465

Holder, Cartridge, Belt, Cal .38, Leather, Black, 6-Round. FSC 8465

Protector, Trousers, Pistol Holster. FSC 8465

Belt, Man's, Waist, Blue 334 (Army Band Uniform). FSC 8405

Belt, All-Weather, Coat, Men's. FSC 8405

Case, Handcuffs, Leather, Black. FSC 8465

Case, Field, First Aid Dressing, Leather (Military Police). FSC 8465

Necklace, Personnel, Identification Tag. FSC 8465

Bandoleer, 200 Round Magazine (M249 Machine Gun). FSC 1305

Cover, Music Carrying Pouch: Embroidered. FSC 7720

Belt, Security Police, 2-1/4-Inch-Wide. FSC 8465

Bag, Pilot Relief (Male). FSC 8105 


\section{C.6 ISO Standards 46}

The ISO standards have been organized as follows:

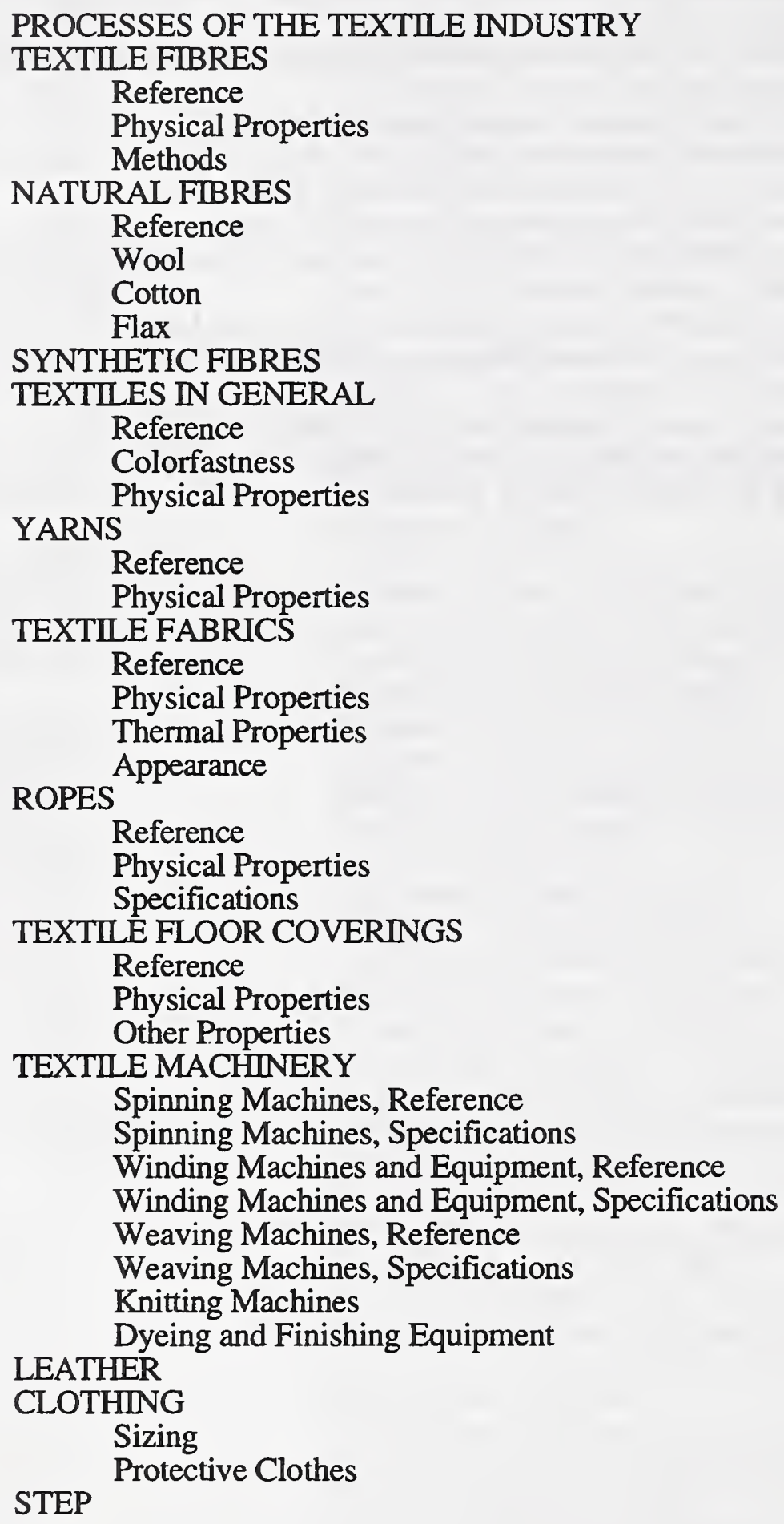

${ }^{46}$ ISO Catalogue 1994. pp. 85, 210-222. 1994. 
PROCESSES OF THE TEXTILE INDUSTRY

ISO 4921:1993

Knitting-basic concepts-Vocabulary. Bilingual Edition. TC 38/SC 20.

\section{TEXTILE FIBERS}

Reference

ISO 8159:1987

Physical Properties

ISO 1973:1976

ISO $6741-1$ to $4: 1989$

ISO 6989:1981

Methods

ISO 1130:1975

ISO 1833:1977

ISO 5088:1976

ISO 5090:1977

Reference

ISO 6938:1984

Wool

ISO 137:1975

ISO 920:1976

ISO 1136:1976

ISO 2646:1974

ISO 2647:1973
Textiles-Morphology of Fibres and Yarns-Vocabulary. Bilingual Edition. TC 38.

Textiles-Determination of Linear Density of Fibres-Gravimetric Method. TC 38/SC 6.

Textiles-Fibres and Yarns-Determination of Commercial Mass of Consignments (4 parts). TC 38.

Part 1: Mass Determination and Calculations.

Part 2: Methods for Obtaining Laboratory Samples.

Part 3: Specimen Cleaning Procedures.

Part 4 : Values Used for the Commercial Allowances and the Commercial Moisture Regains.

Textile Fibres-Determination of length and length distribution of staple fibres (by measurement of single fibres). TC 38/SC 6 .

Textile fibres-Some Methods of Sampling for Testing. TC 38/SC 6.

Textile-Binary Fibre Mixtures-Quantitative Chemical Analysis. TC 38.

Amendment 1:1980 to ISO 1833:1977

Textiles-Ternary Fibre Mixtures-Quantitative Analysis. TC 38/SC 6.

Textiles-Methods for the Removal of Non-Fibrous Matter Prior to Quantitative Analysis of Fibre Mixtures. TC 38.

\section{NATURAL FIBERS}

Textiles-Natural Fibres-Generic Names and Definitions. TC 38.

Wool-Determination of Fibre Diameter-Projection Microscope Method. TC 38/SC 6.

Wool-Determination of Fibre Length (Barbe and Hauter) Using a Comb Sorter. TC 38/SC 6.

Wool-Determination of Mean Diameter of Fibres-Air

Permeability Method. TC 38/SC 6.

Wool-Measurement of the Length of Fibres Processed on the Worsted System, Using a Fibre Diagram Machine. TC 38/SC 6. Wool-Determination of Percentage of Medullated Fibres by the Projection Microscope. TC 38/SC 6. 
ISO 2648:1974

ISO 2913:1975

ISO 2915:1975

ISO 2916:1975

ISO 3072:1975

ISO 3073:1975

Cotton

ISO 2403:1972

ISO $3060: 1974$

ISO 4911:1980

ISO 4912:1981

ISO 4913:1981

ISO $8115: 1986$

ISO 10306:1993

Flax

ISO 2370:1980

ISO 2076:1989

ISO 5079:1977
ISO 3074:1975

Wool-Determination of Fibre Length Distribution ParametersElectronic Method. TC 38/SC 6.

Wool-Colorimetric Determination of Cystine Plus Cystine in Hydrolysates. TC 38.

Wool-Determination of Cysteric Acid Content of Wool

Hydrolysates by Paper Electrophoresis and Colorimetry. TC 38 .

Wool-Determination of Alkalai Content. TC 38.

Wool-Determination of Solubility in Alkalai. TC 38.

Wool-Determination of Acid Content. TC 38.

Wool-Determination of Dichloromethane-Soluble Matter in

Combed Sliver. TC 38.

Textiles-Cotton Fibres-Determination of Micronaire Value. TC 38/SC 6.

Textiles-Cotton Fibres-Determination of Breaking Tenacity of

Flat Bundles. TC 38/SC 6.

Textiles-Cotton Fibres-Equipment and Artificial Lighting for Cotton Classing Rooms. TC 38/SC 6.

Textiles-Cotton Fibres-Evaluation of Maturity-Microscopic Method. TC 38/SC 6.

Textiles-Cotton Fibres-Determination of Length (Span Length) and Uniformity Index. TC 38/SC 6.

Cotton Bales-Dimensions and Density. TC 72/SC 1.

Textiles-Cotton Fibres-Evaluation of Maturity by the Air Flow Method. TC 38/SC 6.

Textiles-Determination of Fineness of Flax FibresPermeametric Methods. TC 38/SC 6.

\section{SYNTHETIC FIBRES}

Textiles-Man-Made Fibres--Generic Names. TC 38.

Textiles-Man-Made Fibres-Determination of Breaking Strength and Elongation of Individual Fibres. TC 38/SC 6.

\section{TEXTILES IN GENERAL}

\section{Reference}

ISO 139:1973

ISO 1144:1973

ISO $2947: 1973$

ISO 3758:1991

ISO 4880:1994
Textiles-Standards Atmospheres for Conditioning and Testing. TC 38.

Textiles-Universal System for Designating Linear Density (Tex Systems). TC 38.

Textiles-Integrated Conversion Table for Replacing Traditional

Yarn Numbers by Rounded Values in the Tex System. TC 38.

Textiles-Care Labeling Code Using Symbols. TC 38/SC 11.

Burning Behavior of Textiles and Textiles Products-Vocabulary. Bilingual Edition. TC 38/SC 19.

Amendment 1:1992 to ISO 4880:1984.

Amendment 2:1993 to ISO 4880:1984. 
ISO 4915:1991

ISO 4916:1991

ISO 5089:1977

ISO 6330:1984

ISO 6348:1990

ISO 7769:1990

ISO 7770:1990

ISO/TR 7248:1985

Colorfastness

ISO 105-A01 to Z02:1978-93
Textiles-Stitch Types-Classification and Terminology.

Bilingual Edition. TC 38.

Textiles-Seam Types-Classification and Terminology.

Bilingual Edition. TC 38.

Textiles-Preparation of Laboratory Test Samples and Text

Specimens for Chemical Testing. TC 38.

Textiles-Domestic Washing and Drying Procedures for Textiles

Testing. TC 38/SC 2.

Textiles-Determination of Mass-Vocabulary. TC 38.

Textiles-Method for Assessing the Appearance of Creases in

Durable Press Products After Domestic Washing and Drying.

(Revision of ISO 7769:1985). TC 38/SC 2.

Textiles-Method for Assessing the Appearance of Seams in

Durable Press Products After Domestic Washing and Drying. TC 38/SC 2.

Fire Data-Collection and Presentation System

Textiles-Tests for Colour Fastness (69 parts). TC 38/SC 1.

Part 1: Low Thermal Resistance.

Part 2: High Thermal Resistance.

Part A01: General Principles of Testing.

Part A02: Grey Scale for Assessing Change in Color.

Part A03: Grey Scale for Assessing Staining.

Part A04: Method for the Instrumental Assessment of the Degree of Staining of Adjacent Fibers.

Part B01: Colour Fastness to Light: Daylight.

Part B02: Colour Fastness to Artificial Light: Xenon Arc Fading.

Part B03: Colour Fastness to Weathering: Outdoor Exposure.

Part B04: Colour Fastness to Weathering: Xenon Arc.

Part B05: Detection and Assessment of Photochromism.

Part B06: Colour Fastness to Artificial Light at High

Temperatures: Xenon Arc Fading Lamp Test.

Part C01: Colour Fastness to Washing: Test 1.

Part C02: Colour Fastness to Washing: Test 2.

Part C03: Colour Fastness to Washing: Test 3.

Part C04: Colour Fastness to Washing: Test 4.

Part C05: Colour Fastness to Washing: Test 5.

Part C06: Colour Fastness to Domestic and Commercial Laundering.

Part D01: Colour Fastness to Dry Cleaning.

Part D02: Colour Fastness to Rubbing: Organic Solvents.

Part E01: Colour Fastness to Water.

Part E02: Colour Fastness to Sea Water.

Part E03: Colour Fastness to Chlorinated Water (SwimmingBath Water).

Part E04: Colour Fastness to Perspiration.

Part E05: Colour Fastness to Spotting: Acid.

Part E06: Colour Fastness to Spotting: Alkali.

Part E07: Colour Fastness to Spotting: Water.

Part E08: Colour Fastness to Water: Hot Water.

Part E09: Colour Fastness to Potting.

Part E11: Colour Fastness to Steaming.

Part E12: Colour Fastness to Milling: Alkaline Milling. 
ISO 105 (con't)

Part E13: Colour Fastness to Acid-Felting: Severe.

Part E14: Colour Fastness to Acid-Felting: Mild.

Part F: Standard Adjacent Fabrics.

Part F10: Specification for Adjacent Fabric: Mulifibre.

Part G: Colour Fastness to Atmospheric Contaminants.

Part G01: Colour Fastness to Nitrogen Oxides.

Part G02: Colour Fastness to Burnt-Gas Fumes.

Part G03: Colour Fastness to Ozone in the Atmosphere.

Part G04: Colour Fastness to Oxides of Nitrogen in the Atmosphere for High Humidities.

Part J01: Measurement of Colour and Colour Differences.

Part J02: Method for the Instrumental Assessment of Whiteness.

Part N: Colour Fastness to Bleaching Agencies.

Part N01: Colour Fastness to Bleaching : Hypochlorite.

Part N02: Colour Fastness to Bleaching : Peroxide.

Part N03: Colour Fastness to Bleaching : Sodium Chlorite (Mild).

Part N04: Colour Fastness to Bleaching : Sodium Chlorite (Severe).

Part N05: Colour Fastness to Stoving.

Part P: Colour Fastness to Heat Treatments.

Part P01: Colour Fastness to Dry Heat (Excluding Pressing).

Part P02: Colour Fastness to Pleating : Steam Pleating.

Past S: Colour Fastness to Vulcanizing.

Part S01: Colour Fastness to Vulcanization : Hot Air.

Part S02: Colour Fastness to Vulcanization : Sulfur Monochloride.

Part S03: Colour Fastness to Vulcanization : Open Steam.

Part X01: Colour Fastness to Carbonizing : Aluminum Chloride.

Part X02: Colour Fastness to Carbonizing : Sulfuric Acid.

Part X04: Colour Fastness to Mercerizing.

Part X05: Colour Fastness to Organic Solvents.

Part X06: Colour Fastness to Soda Boiling.

Part X07: Colour Fastness to Cross-Dyeing : Wool.

Part X08: Colour Fastness to Degumming.

Part X09: Colour Fastness to Formaldehyde.

Part X10: Assessment of Migration of Textile Colours into Polyvinyl Chloride Coating.

Part X11: Colour Fastness to Hot Pressing.

Part X12: Colour Fastness to Rubbing.

Part X13: Colour Fastness of Wool Dyes to Processes Using Chemical Means for Creasing, Pleating and Setting.

Part X14: Colour Fastness to Acid Chlorination of Wool: Sodium Dichloroisocyanurate.

Part Z: Colorant Characteristics.

Part Z01: Colour Fastness to Metals in the Dye-Bath : Chromium Salts.

Part Z02: Colour Fastness to Metals in the Dye-Bath : Iron and Copper.

Physical Properties

ISO 2960:1974

ISO 3071:1980
Textiles-Determination of Bursting Strength and Bursting Distension-Diaphragm Method. TC 38.

Textiles-Determination of $\mathrm{pH}$ of the Aqueous Extract. TC 38 . 
ISO 3175: 1979

ISO 3998: 1977

ISO 5077:1984

ISO $5085-1$ to $2: 1989-90$

ISO/TR 8091:1983

ISO/TR 9240:1992

ISO 11092:1993
Textiles-Determination of Dimensional Change on Dry Cleaning in Perchlorethylene-Machine Method. TC 38/SC 2.

Textiles-Determination of Resistance to Certain Insect Pests. TC 38.

Textiles-Determination of Dimensional Change in Washing and Drying. TC 38/SC 2.

Textiles-Determination of Thermal Resistance (2 Parts). TC 38.

Textiles-Twist Factor Related to the Tex System. TC 38.

Textiles-Design of Apparel for Reduced Fire Hazard. TC 38/SC 19.

Textiles-Physiological Effects-Measurement of Thermal and Water-Vapour Resistance Under Steady-State Conditions (Sweating Guarded-Hotplate Test). TC 38.

\section{YARNS}

Reference
ISO 1139:1973

ISO 2:1973

ISO 8159:1987

ISO 8160:1987

ISO 10132:1993

ISO 10290:1993

Physical Properties

ISO 2061:1973

ISO 2062:1993

ISO 2060:1972

ISO 6939:1988

ISO $6741-1$ to $4: 1987-89$

Textiles-Designations of Yarns. TC 38.

Textiles-Designation of the Direction of Twist in Yarns and Related Products. TC 38/SC 6.

Textiles-Morphology of Fibres and Yarns-Vocabulary. Bilingual Edition. TC 38.

Textiles-Textured Filament Yarns-Vocabulary. Bilingual Edition. TC 38.

Textiles-Textured Filament Yarns-Definitions. Bilingual Edition. TC 38/SC 5.

Textiles-Cotton Yarns-Specifications. TC 38/SC 22.

Textiles-Determination of Twist in Yarns-Skein Method. TC 38/SC 6.

Textiles-Yarns from Packages-Determination of Single-End Breaking Force and Elongation at Break. TC 38/SC 5.

Textiles - Yarns from Packages-Determination of Linear Density (Mass per Unit Length)-Skein Method. TC 38/SC 5.

Textiles-Yarns from Packages-Method of Test for Breaking Strength of Yarn by the Skein Method. TC 38/SC 5.

Textiles-Fibres and Yarns-Determination of Commercial Mass of Consignments (4 parts). TC 38.

Part 1: Mass Determination and Characteristics.

Part 2: Methods for Obtaining Laboratory Samples.

Part 3: Specimen Cleaning Procedures.

Part 4: Values Used for the Commercial Allowances and the Commercial Moisture Regains.

\section{TEXTILE FABRICS}

Reference

ISO 2959:1973

ISO 3572:1976
Textiles-Woven Fabric Descriptions. TC 38/SC 20.

Textiles-Weaves-Definitions of General Terms and Basic Weaves. TC 38/SC 20. 
ISO $3759: 1984$

ISO $7211-1$ to $6: 1984$

ISO 8498: 1990

ISO 8499:1990

ISO 9092:1988

ISO 9354:1989

ISO 11224:1993

Physical Properties

ISO 675:1979

ISO $811: 1981$

ISO 2649:1974

ISO 3005: 1978

ISO 3801:1977

ISO 3932: 1976

ISO 3933:1976

ISO 4920:1981

ISO 5081:1977

ISO 5082:1982

ISO 5084:1977

ISO 7771:1985

ISO 9073-1 to 5:1989

ISO 9290:1990
Textiles-Preparation, Marking and Measuring of Fabric Specimens and Garments in Tests for Determination of Dimensional Change. TC 38/SC 2.

Textiles-Woven Fabric-Construction-Method of Analysis (4 parts). TC 38/SC 20.

Part 1: Methods for the Presentation of a Weave Diagram and Plans for Drafting, Denting, and Lifting.

Part 2: Determination of Number of Threads per Unit Length.

Part 3: Determination of Crimp of Yarn in Fabric.

Part 4: Determination of Twist in Yarn Removed from Fabric.

Woven Fabrics-Description of Defects-Vocabulary. Bilingual Edition. TC 38/SC 20.

Knitted Fabrics-Description of Defects-Vocabulary. Bilingual Edition. TC 38/SC 20.

Textiles-Nonwovens-Definition. Bilingual Edition. TC 38.

Textiles-Weaves-Coding System and Examples. TC 38/SC 20.

Textiles-Nonwovens-Web Formation and Bonding-

Vocabulary. TC 38.

Textiles-Woven Fabrics-Determination of Dimensional Change on Commercial Laundering Near the Boiling Point. TC 38/SC 2.

Technical Corrigendum 1:1980 to ISO 675:1979.

Textile Fabrics-Determination of Resistance to Water

Penetration-Hydrostatic Pressure Test. TC 38/SC 2.

Wool-Determination of Short-Term Irregularity of Linear

Density of Slivers, Rovings and Yarns, by Means of an Electronic Evenness Tester. TC 38/SC 6.

Textiles-Woven Fabrics-Determination of Mass per Unit Length and Mass per Unit Area. TC 38.

Textiles-Woven Fabrics-Measurement of Width of Pieces. TC 38.

Textiles-Woven Fabrics-Measurement of Length of Pieces.

TC 38.

Textiles-Determination of Resistance to Surface Wetting (Spray

Test) of Fabrics. TC 38/SC 2.

Textiles-Woven Fabrics-Determination of Breaking Strength and Elongation (Strip Method). TC 38.

Textiles-Woven Fabrics-Determination of Breaking StrengthGrab Method. TC 38.

Textiles-Determination of Thickness of Woven and Knitted

Fabrics (Other than Textile Floor Coverings). TC 38.

Textiles-Determination of Dimensional Changes of Fabrics Induced by Cold-Water Immersion. TC 38/SC 2.

Textiles-Test Methods for Nonwovens (4 parts). TC 38.

Part 1: Determination of Mass per Unit Area.

Part 2: Determination of Thickness.

Part 3: Determination of Tensile Strength and Elongation.

Part 4: Determination of Tear Resistance.

Textiles-Woven Fabrics-Determination of Tear Resistance by the Falling Pendulum Method. TC 38. 
ISO 9865:1991

Thermal Properties

ISO 6940:1984

ISO 6941:1984

ISO 9866-1 to 2:1991

ISO $10047: 1993$

Appearance

ISO 2313:1972

ISO 7768:1985

ISO 9867:1991

Reference

ISO 1968: 1973

ISO 3505:1975

Physical Properties

ISO 2307:1990

ISO 3090:1974

Specifications

ISO 9554:1991

ISO 1969:1990

ISO 1140:1990

ISO 1141:1990

ISO 1181:1990

ISO 1346: 1990

ISO 4167:1979

ISO 4878:1991
Textiles-Determination of Water Repellancy of Fabrics by the Bundesmann Rain-Shower Test. TC 38/SC 2.

Textiles Fabrics-Burring Behaviour-Determination of Ease of Ignition of Vertically Oriented Specimens. TC 38/SC 19.

Amendment 1:1993 to ISO 6940:1984.

Textile Fabrics-Burning Behaviour-Determination of Flame Spread Properties of Vertically Oriented Specimens. TC 38/SC 19.

Amendment 1:1993 to ISO 6941:1984.

Textiles-Effect of Dry Heat on Fabrics Under Low Pressure (2 parts). TC 38/SC 2.

Part 1: Procedure for Dry-Heat Treatment of Fabrics.

Part 2: Determination of Dimensional Change in Fabrics Exposed to Dry Heat.

Textiles-Determination of Surface Burning Time of Fabrics.

Textiles-Determination of the Recovery from Creasing of a Horizontally Folded Specimen of Fabric by Measuring the Angle Recovery. TC 38.

Textiles-Method for Assessing the Appearance of Durable Fabrics After Domestic Washing and Drying.

Textiles-Evaluation of Wrinkle Recovery of the Wrinkle

Recovery of Fabrics-Appearance Method. TC 38/SC 2.

\section{ROPES}

Ropes and Cordage-Vocabulary. Bilingual Edition. TC 38. Ropes an Cordage-Equivalence Between Natural Fibre Ropes and Man-Made Fibre Ropes for Use in the Mooring of Vessels.

Ropes-Determination of Certain Physical and Mechanical Properties. TC 38.

Ropes and Cordage-Netting Yarns-Determination of Change in Length After Immersion in Water. TC 38.

Fibre Ropes-General Specification. TC 38.

Ropes-Polyethylene-Specification. TC 38.

Ropes-Polyamide-Specification. TC 38.

Ropes-Polyester-Specification. TC 38.

Ropes-Manila and Sisal-Specification. TC 38.

Ropes-Polypropylene-Specification. TC 38.

Ropes and Cordage-Sisal Agricultural Twines. TC 38.

Textiles-Flat Woven Webbing Slings Made of Man-Made

Fibres. TC 38. 


\section{TEXTILE FLOOR COVERINGS}

Reference

ISO 1957:1986

ISO 2424:1992

ISO 5086:1977

ISO/TR 6131:1986

ISO 6347:1989

Physical Properties

ISO 1763:1986

ISO $1765: 1986$

ISO $1766: 1986$

ISO 2094:1986

ISO 2549:1972

ISO 2551:1981

ISO 3018:1974

ISO 3415:1986

ISO 3416:1986

ISO/TR 4918:1990

ISO 4919:1978

ISO 8543:1986

ISO 10833:1992

ISO 10834:1992

ISO 2550:1972

Other Properties

ISO/TR 6356:1982

ISO 6925:1982
Machine-Made Textile Floor Coverings-Sampling and Cutting Specimens for Physical Tests. TC 38/SC 12.

Textile Floor Coverings-Vocabulary. Bilingual Edition. TC 38/SC 12.

Textile Floor Coverings-Hand-Knotted Carpets-Sampling and Selection of Areas of Test. TC 38/SC 12.

Textile Floor Coverings-Tetrapod Walker Apparatus-

Constructional Details and Instructions for Use. TC 38/SC 12.

Textile Floor Coverings-Consumer Information. TC 38/SC 12.

Carpets-Determination of Number of Tufts and/or Loops per Unit Length and per Unit Area. TC 38/SC 12.

Machine-Made Textile Floor Coverings-Determination of Thickness. TC 38/SC 12.

Textile Floor Coverings-Determination of Thickness of Pile above the Substrate. TC 38/SC 12.

Textile Floor Coverings-Determination of Thickness Loss Under Dynamic Loading. TC 38/SC 12.

Textile Floor Coverings-Hand-Knotted Carpets-Determination of Tuft Length Above the Woven Ground.

Technical Corrigendum 1:1990 to ISO 2549:1972.

Machine-Made Textile Floor Coverings-Determination of

Dimensional Changes Due to the Effects of Varied Water and Heat Conditions. TC 38/SC 12.

Textile Floor Coverings-Rectangular Textile Floor CoveringsDetermination of Dimensions. TC 38/SC 12.

Textile Floor Coverings-Determination of Thickness Loss After Brief, Moderate Static Loading. TC 38/SC 12.

Textile Floor Coverings-Determination of Thickness Loss After Prolonged, Heavy Static Loading. TC 38/SC 12.

Textile Floor Coverings-Determination of Wear-Castor Chair Test. TC 38/SC 12.

Textile Floor Coverings-Determination of Tuft Withdrawal

Force. TC 38/SC 12.

Textile Floor Coverings-Methods for Determination of Mass.

TC 38/SC 12.

Textile Floor Coverings-Determination of Mechanical Damage at a Cut Edge-Modified Vetterman Drum Tester Method. TC 38/SC 12 .

Textile Floor Coverings-Non-Destructive Measurement of Pile Thickness Above the Backing-WRONZ Gauge Method. TC 38/SC 12.

Textile Floor Coverings-Hand-Made Carpets-Determination of Types of Knots. TC 38/SC 12.

Textile Floor Coverings-Assessment of Static Electrical Propensity-"Walking" Test. TC 38/SC 12.

Textile Floor Coverings-Burning Behavior-Tablet Test at Ambient Temperature. TC 38/SC 19. 
ISO/TR 9405:1990

ISO/TR 10361:1990

ISO 3799:1976

ISO 5232:1988

ISO 9902:1993

Textile Floor Coverings-Assessment of Changes in Appearance. TC 38/SC 12.

Textile Floor Coverings-Production of Changes in Appearance by Means of a Vetterman Drum and Hexapod Tumbler Testers. TC 38/SC 12.

\section{TEXTILE MACHINERY}

Textile Machinery and Accessories-Hydraulic Lubrication Fittings for Textile Machinery. TC 72/SC 1.

Graphical Symbols for Textile Machinery. Bilingual Edition. TC 72.

Textile Machinery Accessories-Determination of Sound Pressure Levels and Sound Power Levels Emitted by Textile MachinesEngineering and Survey Methods. TC 72.

Spinning Machines, Reference ISO 1809:1977

ISO 2187:1990

ISO 2205:1975

ISO 5234: 1980

ISO 6173:1982

ISO 8114:1990

ISO $9947: 1991$

Spinning Machines, Specifications

ISO 92:1976

ISO $93-1$ to $3: 1978-82$

ISO 94:1982

ISO $96-1$ to $2: 1992$

ISO 98:1977

ISO 341:1976
Textile Machinery and Accessories-Types of Formers for Yarn Packages-Nomenclature. Trilingual Edition. TC 72/SC 2. Spinning Preparatory Machinery, Spinning and Doubling (Twisting) Machinery - List of Equivalent Terms. Trilingual Edition. TC 72/SC 1.

Textile Machinery and Accessories-Drafting Arrangements for Spinning Machines-Terminology. Trilingual Edition. TC 72. Textile Machinery and Accessories-Metallic Card ClothingTerms and Definitions. Trilingual Edition. TC 72. Open-End Spinning Machines-Vocabulary. TC 72/SC 1. Textile Machinery and Accessories-Spindles for Ring-Spinning and Doubling Machines-List of Equivalent Terms. Trilingual Edition. TC 72/SC 1.

Textile Machinery and Accessories-Two-for-One TwistersVocabulary. Bilingual Edition. TC 72/SC 1.
Textile Machinery and Accessories-Spinning MachineryDefinition of Side (Left or Right). TC 72/SC 1.

Textile Machinery and Accessories-Cylindrical Sliver Cans (3 parts). TC 72/SC 1.

Part 1: Main Dimensions.

Part 2: Spring Bottoms.

Part 3: Packaging Slive Cans (Press Cans).

Textile Machinery and Accessories-Spindle Gauges for RingSpinning and Ring-Doubling Frames. TC 72/SC 1.

Textile Machinery and Accessories-Rings and Travellers for Ring Spinning and Ring Doubling Frames (2 parts). TC 72/SC 1.

Part 1: T-Rings and Their Appropriate Travellers.

Part 2: $\mathrm{HZCH}-, \mathrm{HZ}$ - and J-Rings and Their Appropriate Travellers.

Textile Machinery and Accessories-Spinning Preparatory and Spinning Machinery-Covering Characteristics of Top Rollers. TC 72/SC 1.

Textile Machinery and Accessories-Cotton Spinning Machinery-Working Width. TC 72/SC 1. 
ISO $342: 1983$

ISO $2572: 1982$

ISO 3464:1977

ISO 4105:1978

ISO 5233:1978

ISO 5235:1977

ISO 6170:1983

ISO $6171: 1982$

ISO 9903:1991

ISO 9904:1989
Textile Machinery and Accessories-Worsted and Woolen Cards-Width of Cylinder and Width on the Wire. TC 72/SC 1. Textile Machinery and Accessories-Card Gauges. TC 72. Textile Machinery and Accessories-Bearings for Bottom Rollers and Allied Dimensions-Caps with Central Nose and Caps Side Lugs. TC 72/SC 1.

Textile Machinery and Accessories-Wires for Flexible Card Clothing. TC 72/SC 1.

Textile Machinery and Accessories-Bottom Fluted Rollers for Drafting Systems. TC 72/SC 1.

Textile Machinery and Accessories-Ring-Spinning Frames and Speedframes-Top and Bottom Aprons. TC 72/SC 1.

Spinning Machinery-Condenser Rubbers for Cards. TC 72/SC 1 .

Textile Machinery and Accessories-Bead Wires and Corresponding Grooves for Cards-Main Types and Dimensions. TC 72/SC 1.

Textile Machinery and Accessories-Wires for Metallic Card Clothing. TC 72/SC 1.

Textile Machinery and Accessories-Steel Pins for Spinning Preparatory and Spinning Machinery. TC 72/SC 1.

Winding Machines and Equipment, Reference

ISO 141:1976

ISO 476:1982

ISO $477: 1982$

ISO 1037:1982

ISO 5238-1 to 2:1983

ISO 5239:1980

ISO 5240:1978

ISO 8116-1 to 9:1985-91
Textile Machinery and Accessories-Pirn Winders and Cross Winders-Definition of left and right sides. TC 72/SC 2. Textile Machinery and Accessories-Pirn Winding MachinesVocabulary. Trilingual Edition. TC 72/SC 2.

Textile Machinery and Accessories-Cones and Cheese Winding Machines-Vocabulary. Trilingual Edition. TC 72/SC 2.

Textile Machinery and Accessories-Beams for Dyeing Slivers and Yarn-Terminology and Main Dimensions. TC 72/SC 4. Textiles Machinery and Accessories-Packages of yarns and Intermediate Products ( 2 parts). TC 72/SC 2.

Part 1: Terminology. Trilingual Edition.

Part 2: Forms of Winding. Trilingual Edition.

Textile Machinery and Accessories-Winding-Basic Terms. Trilingual Edition. TC 72.

Textile Machinery and Accessories-Warp Creels-Terminology and Main Dimensions. TC 72/SC 2.

Textile Machinery and Accessories-Beams for Winding (7 parts). TC 72/SC 2.

Part 1: Vocabulary. Trilingual Edition.

Part 2: Warper's Beams-Terminology and Main Dimensions.

Part 3: Weaver's Beams-Terminology and Main Dimensions.

Part 4: Quality Classification of Flanges for Weaver's Beams, Warper's Beams and Sectional Beams.

Part 5: Sectional Beams for Warp Knitting-Terminology and Main Dimensions.

Part 6: Beams for Ribbon Weaving and Ribbon KnittingTerminology and Main Dimensions.

Part 9: Dyeing Beams for Textile Fabrics. 
Winding Machines and Equipment. Specifications

ISO 111:1978

ISO 112:1983

ISO 324:1978

ISO $344: 1981$

ISO 368:1991

ISO 574:1979

ISO 575:1978

ISO 1472:1977

ISO 1946:1976

ISO 2013:1983

ISO $3914-1$ to $4: 1981-89$

ISO 5237:1978

ISO 6169:1982

ISO 6175:1983

ISO 8489-1:1985

ISO 10458:1993
Textile Machinery and Accessories-Cones for Yarn Winding (Cross Wound)-Half Angle of the Cone 4 degrees 20'. TC 72/SC 2.

Textile Machinery and Accessories-Cones for Yarn Winding (Cross Wound)-Half Angle of the Cone 3 degrees 30'. TC 72/SC 2.

Textile Machinery and Accessories-Cones for Cross Winding for Dyeing Purposes-Half Angle of the Cone 4 degrees 20'. TC 72/SC 2.

Textile Machinery and Accessories-Spinning Machines-Flyer Bobbins. TC 72.

Spinning Preparatory, Spinning and Doubling (Twisting) Machinery-Tubes for Ring-Spinning, Doubling and Twisting Spindles, Taper 1:38 and 1:64. TC 72/SC 1.

Textile Machinery and Accessories-Perforated Cylindrical Tubes for Cheese Dyeing. TC 72.

Textile Machinery and Accessories-Transfer Cones-Half Angle of the Cone 4 degrees 20. TC 72/SC 2.

Textile Machinery and Accessories-Cylindrical Tubes for DrawTwisters-Dimensions and Permissible Run-Out. TC 72/SC 1. Textile Machinery and Accessories-Condenser Bobbins for Woollen Spinning-Dimensions. TC 72/SC 1.

Textile Machinery and Accessories-Beams-Method of Measuring Variations of Form and Position. TC 72/SC 1. Textile Machinery and Accessories-Cylindrical Tubes (4 parts). TC $72 / \mathrm{SC} 1$.

Part 1: Recommended Values of Inner Diameters and Lengths.

Part 2: Tubes for Open-End Spinning Machines.

Part 3: Tubes for Tape Yarns.

Part 4: Tubes for Textured Yarns.

Textile Machinery and Accessories-Cones for Yarn Winding (Cross Wound)-Half Angle of the Cone 5 degrees 57'. TC 72/SC 2.

Textile Machinery and Accessories-Flanged Bobbins for Doubling and Twisting. TC 72/SC 1.

Textile Machinery and Accessories-Recommended Profile Threads for Weaver's Beams. TC 72/SC 2.

Textile Machinery and Accessories-Cones for Cross-Wound Winding-Part 1: Values of Half-Angles, Lengths and Large Inner Diameters. TC 72/SC 2.

Textile Machinery-Square Bars for Winding Devices Relating to Dyeing and Finishing Machines-Dimensions. TC 72/SC 4.
Weaving Machines, Reference ISO 108:1976

ISO 142:1976

ISO 1586:1977
Textile Machinery and Accessories-Weaving Looms-Definition of Left and Right Sides. TC 72/SC 3.

Textile Machinery-Working Widths of Weaving Machines. TC 72/SC 2.

Textile Machinery and Accessories-Shuttles-Terms and Designation in Relation to the Position of the Shuttle Eye. Trilingual Edition. TC 72/SC 3. 
ISO $1865: 1977$

ISO 2544:1975

ISO $7506: 1984$

ISO $5247-1$ to $3: 1983$

ISO 6177:1986
Textile Machinery and Accessories-Serrated Bars for Mechanical Warp Stop Motions-Designations of Dimensions, and

Dimensions of Cross-Section. Trilingual Edition. TC 72/SC 3.

Textile Machinery and Accessories-Warping Machinery-

Preparation of Warp for Weaving-Vocabulary. Trilingual Edition. TC 72/SC.

Textile Machinery and Accessories-Numbering of Harnesses for Drawing-in on Jacquard Machines. TC 72/SC 3.

Textile Machinery and Accessories-Weaving Machines-

Classification and Vocabulary ( 3 parts). Trilingual Edition. TC 72/SC 3.

Part 2: Accessories-Vocabulary.

Part 3: Parts of the Machine-Vocabulary. Bilingual Edition.

Textile Machinery-Cloth Rollers-Terminology and Main

Dimensions. TC 72/SC 3.

Weaving Machines, Specifications

ISO 109:1982

ISO $143: 1977$

ISO $227: 1978$

ISO 363:1992

ISO $364: 1983$

ISO $365: 1982$

ISO $366-1$ to $4: 1988-92$

ISO 441:1978

ISO 568:1976

ISO 569:1982

ISO $570: 1982$

ISO 572:1976

ISO 573:1976

ISO 576:1976
Textile Machinery-Working Widths of Weaving Machines. TC 72/SC 3.

Textile Machinery and Accessories-Weft Pirns for Automatic Looms. TC 72/SC 3.

Textile Machinery and Accessories-Single Box Pickers for Centre Tip Shuttles for Automatic Looms and Related Picking Stick Dimensions. TC 72/SC 3.

Textile Machinery and Accessories-Flat Steel Healds with Closed End Loops-Dimensions. TC 72/SC 3.

Textile Machinery and Accessories-Twin Wire Healds with Inset Mail for Jacquard Weaving. TC 72/SC 3.

Textile Machinery and Accessories-Twin Wire Healds with Inset Mail for Jacquard Weaving. TC 72/SC 3.

Textile Machinery and Accessories-Reeds (4 parts). TC 72/SC 3.

Part 1: Pitch Bound Reeds-Dimensions.

Part 2: Metal Reeds with Plate Baulk-Dimensions and Designation.

Part 3: Metal Reeds with Double-Spinning Baulk-Dimensions and Designation.

Part 4: Plastic Bound Metal Reeds-Dimensions and Designation..

Textile Machinery and Accessories-Drop Wires for Mechanical and Electrical Warp Stop Motions. TC 72/SC 3.

Textile Machinery and Accessories-Heald Frames for Single or Double Row Healds-Designation of Dimensions. TC 72/SC 3. Textile Machinery and Accessories-Heald Frames-Coordinated Dimensions in Relation to the Pitch of the Harness. TC 72/SC 3. Textile Machinery and Accessories-Heald Carrying Rods for Healds with Closed "O"-shaped End Loops. TC 72/SC 3. Textile Machinery and Accessories-Shuttle for Pirn Changing Automatic Looms-Dimensions. TC 72/SC 3.

Textile Machinery and Accessories-Dobby Lags and Pegs in Wood, Metal or Other Suitable Material-Dimensions. TC 72/SC 3.

Textile Machinery and Accessories-Paper Patterns for DobbiesDimensions. TC 72/SC 3. 
ISO 1131:1976

ISO 1150:1978

ISO 2012:1976

ISO 2748:1983

ISO 5243:1977

ISO 5245:1977

ISO 5246:1977

ISO 6176:1981

ISO 6457:1982

ISO 8118:1986

ISO 9473:1986

Knitting Machines ISO 7839:1984

ISO $8117: 1986$

ISO $8119-1$ to $3: 1989$

ISO $8121: 1986$

ISO 8122:1988

ISO 8188: 1986

ISO $8640-1$ to $2: 1990$

ISO 10223:1992
Textile Machinery and Accessories-Weft Pirns for Box-Loaders for Automatic Looms-Dimensions of Pirn Tip. TC 72/SC 3. Textile Machinery and Accessories-Closed-End Drop Wires for Mechanical Warp Stop Motions-Designations of Dimensions, and Dimensions of Cross-Section. Trilingual Edition. TC 72/SC 3 .

Textile Machinery and Accessories-Cone Sectional Warping Machines-Maximum Usable Width. TC 72/SC 3.

Textile Machinery and Accessories-Lingoes for Jacquard Weaving. TC 72/SC 3.

Textile Machinery and Accessories-Numbering of Heald Frames in a Loom. TC 72/SC 3.

Textile Machinery and Accessories-Weft Pirns with Rings $(27 \mathrm{~mm}$ and $30 \mathrm{~mm}$ ) for Automatic Winding at the Loom. TC 72/SC 3.

Textile Machinery and Accessories-Ringless Weft Pirns (24mm and $27 \mathrm{~mm}$ ) for Automatic Winding at the Loom. TC 72/SC 3. Textile Machinery-Warp Sizing Machines-Maximum Usable Width. TC 72.

Textile Machinery and Accessories-Heald Carrying Rod for "C" shaped End Loop of Flat Steel Healds-Dimensions. TC 72/SC 3.

Weaving Machines-Temple Cylinders. Trilingual Edition. TC 72/SC 3.

Textile Machinery and Accessories-Strip Steel for Dents of Reed. TC 72/SC 3.

Textile Machinery and Accessories-Knitting MachinesClassification and Vocabulary. Trilingual Edition. TC 72/SC 3. Textile Machinery-Knitting Machines-Nominal Diameters of Circular Machines. TC 72/SC 3.

Textile Machinery and Accessories-Needles for Knitting Machines-Terminology (3 parts). Trilingual Edition. TC 72/SC 3.

Part 1: Latch-Type Needles.

Part 2: Bearded Needles.

Part 3: Compound Needles.

Textile Machinery-Knitting Machines-Nameplate Information. TC 72/SC 3.

Technical Corrigendum 1:1992 to ISO 8121:1986.

Textile Machinery-Knitting Machines-Number of Needles for Circular Knitting Machines of Large Nominal Diameter. TC 72/SC 3.

Textile Machinery and Accessories-Pitches of Knitting Machine Needles. Bilingual Edition. TC 72/SC 3.

Textile Machinery and Accessories-Flat Warp Knitting Machines-Vocabulary (2 parts). TC 72/SC 3. Trilingual Edition Part 1: Driving Mechanisms, Supports and Knitting Elements. Part 2: Warp Let-off, Fabric Take-up and Batching. Textile Machinery-Flat Warp Knitting Machines-Numbering of Guide Bars. TC 72/SC 3. 
Dyeing and Finishing Equipment

ISO 1036:1984

ISO 1505:1993

ISO $1506: 1982$

ISO 5248:1982

ISO 5249:1988

ISO 5250: 1982

ISO $6178: 1983$

ISO 10457:1991

ISO 10459:1992

ISO 1164:1993

ISO 2417:1972

ISO 2418:1972

ISO 2419:1972

ISO 2420:1972

ISO 2588:1985

ISO 2589:1972

ISO 2820:1974

ISO $2821: 1974$

ISO $3376: 1976$

ISO 3377:1975

ISO $3378: 1975$

ISO $3379: 1976$

ISO $3380: 1975$

ISO 4044:1977

ISO 4045: 1977

ISO 4047:1977
Textile Machinery-Dyeing and Finishing Machines-Definition of Left and Right Sides. TC 72/SC 4.

Textile Machinery-Widths Relating to Dyeing and Finishing Machines-Definition and Range of Nominal Widths. TC 72/SC 4.

Textile Machinery-Dyeing, Finishing and Allied MachineryClassification and Nomenclature. Trilingual Edition. TC 72/SC 4.

Textile Machinery and Accessories-Dyeing and Finishing Machinery - Vocabulary for Ancillary Devices. Trilingual Edition. TC 72/SC 4.

Textile Machinery and Accessories-Guide Rollers for Dyeing and Finishing Machinery-Main Dimensions. TC 72/SC 4. Textile Machinery and Accessories-Dyeing and Finishing Machinery - Vocabulary for Stenters. TC 72/SC 4.

Centrifuges-Construction and Safety Rules-Method for the Calculation of the Tangential Stress in the Shell of a Cylindrical Centrifuge Rotor. TC 72/SC 4.

Textile Machinery-Dyeing and Finishing Machines-Nominal Speeds. TC 72/SC 4.

Textile Machinery-Dyeing and Finishing Machines-Designation of Operating Ranges of Component Parts. TC 72/SC 4.

\section{LEATHER}

Leather-Tests for Adhesion of Finish. IULTCS.

Leather-Determination of Absorption of Water. IULTCS.

Leather-Laboratory Samples-Location and Identification.

IULTCS.

Leather - Condition of Test Pieces for Physical Tests. IULTCS.

Leather-Determination of Apparent Density. IULTCS.

Leather-Sampling-Number of Items for a Gross Sample.

IULTCS.

Leather-Physical Testing-Measurement of Thickness.

IULTCS.

Leather-Raw Hides of Cattle and Horses-Method of Trim. TC 120.

Leather-Raw Hides of Cattle and Horses-Preservation by Stack

Salting. TC 120.

Leather-Determination of Tensile Strength and Elongation.

IULTCS.

Leather-Determination of Tearing Load. IULTCS.

Leather-Determination of Resistance to Grain Cracking, and of Crack Index. IULTCS.

Leather-Determination of Distension and Strength of Grain-

Ball Burst Test. IULTCS.

Leather-Determination of Shrinkage Temperature. IULTCS.

Leather-Preparation of Chemical Test Sample. IULTCS.

Leather-Determination of $\mathrm{pH}$. IULTCS.

Leather-Determination of Sulphated Total Ash and Sulphated

Water-Insoluble Ash. IULTCS. 
ISO 4048:1977

ISO $5397: 1984$

ISO 5399:1984

ISO 11640:1993

ISO 11641:1993

ISO 11642:1993

ISO 11645:1993

ISO 11646:1993
ISO 5400:1984

ISO 11643:1993

Leather-Determination of Matter Soluble in Dichloromethane. IULTCS.

Leather-Determination of Nitrogen Content and "Hide

Substance"-Titrimetric Method. IULTCS.

Leather-Determination of Water-Soluble Magnesium SaltsEDTA Titrimetric Method. IULTCS.

Leather-Determination of Total Silicon Content-Reduced

Molybdosilcate Spectrometric Method. IULTCS.

Leather-Tests for Colour Fastness-Colour Fastness to Cycles of to-and-fro Rubbing. IULTCS.

Leather-Tests for Colour Fastness - Colour Fastness to

Perspiration. IULTCS.

Leather-Tests for Colour Fastness-Colour Fastness to Water. IULTCS.

Leather-Tests for Colour Fastness-Colour Fastness of Small Samples to Dry-Cleaning Solutions. IULTCS.

Leather-Heat Stability of Industrial-Glove Leather. IULTCS.

Leather-Measurement of Area. IULTCS.

\section{CLOTHING}

Sizing

ISO 3635:1981

ISO 3638:1977

ISO 3636:1977

ISO $3637: 1977$

ISO 4416:1981

ISO/TR 10652:1991

ISO 4118:1978

ISO 4417:1977

ISO 7070:1982

ISO 5971:1981

ISO $8559: 1989$

Protective Clothing

ISO 2801:1973

ISO 6529:1990

ISO 6530:1990
Size Designation of Clothes-Definitions and Body Measurement Procedure. TC 133.

Size Designation of Clothes-Infants' Garments. TC 133.

Size Designation of Clothes-Men's and Boy's Outerwear Garments.

Technical Corrigendum 1:1990 to ISO 3636:1977.

Size Designation of Clothes-Women's and Girl's Outerwear Garments. TC 133.

Technical Corrigendum 1:1990 to ISO 3637:1977.

Size Designation of Clothes-Women's and Girls' Underwear,

Nightwear, Foundation Garments and Shirts. TC 133.

Technical Corrigendum 1:1990 to ISO 4416:1981.

Standard Sizing Systems for Clothes. TC 133.

Size Designation of Clothes-Gloves. TC 133.

Size Designation of Clothes-Headwear. TC 133.

Size Designation of Clothes-Hosiery. TC 133.

Size Designation of Clothes-Pantyhose. TC 133.

Garment Construction and Anthropometric Surveys-Body

Dimensions. TC 133.

Clothing for Protection Against Heat and Fire-General

Recommendations for Users and for Those in Charge of Such Users. TC 94/SC 13.

Protective Clothing-Protection Against Liquid ChemicalsDetermination of Resistance of Air-Impermeable Materials to Permeation by Liquids. TC 94/SC 13.

Protective Clothing-Protection Against Liquid ChemicalsDetermination of Resistance of Air-Impermeable Materials to Permeation by Liquids. TC 94/SC 13. 
ISO 6942:1993

ISO $8096-1$ to $3: 1988-89$

ISO 8194:1987

ISO 9150:1990

ISO 9185:1988

ISO/TR 11079:1993

ISO $10303-1$ to 203:1994-95
Clothing for Protection Against Heat and Fire-Evaluation of Thermal Behaviour of Materials and Material Assemblies When Exposed to a Source of Radiant Heat. TC 94/SC 13.

Rubber- or Plastics-Coated Fabrics for Water-Resistant Clothing Specification (3 parts). TC 45.

Part 1: PVC-Coated Fabrics.

Technical Corrigendum 1:1991 to ISO 8096-1:1989.

Part 2: Polyurethane- and Silicone Elastomer-Coated Fabrics.

Part 3: Natural Rubber- and Synthetic Rubber-Coated Fabrics.

Radiation Protection-Clothing for Protection Against Radioactive Contamination-Design, Selection, Testing and Use. TC 85/SC 2.

Protective Clothing-Determination of Behaviour of Materials on Impact of Small Splashes of Molten Metal. TC 94/SC 13.

Protective Clothing-Assessment of Resistance of Materials to Molten Metal Splash. TC 94/SC 13.

Evaluation of Cold Environments-Determination of Requisite Clothing Insulation (IREC). TC 159/SC 5.

\section{STEP47}

Part 1: Overview and Fundamental Principles.

Part 11: EXPRESS Language and Reference Manual.

Part 21: Physical File, Exchange Structure Working Format, Active Transfer.

Part 41: Fundamentals of Product Description and Support.

Part 42: Geometry and Topology Representations.

Part 43: Representation Specialization.

Part 44: Product Structure Configuration.

Part 46: Visual Presentation.

Part 101: Draughting.

Part 201: Explicit Draughting.

Part 203: Configuration-Controlled Design.

47 This listing includes only the STEP initial release. There are many other parts in some stage of the development and approval process. 


\section{C.7 NFPA Apparel Standards 48}

The following are performance specifications for clothing to protect against hazardous environments. Most of them relate to fire fighting. The standards are listed in numerical order.

NFPA 1971

NFPA 1972

NFPA 1973

NFPA 1974

NFPA 1975

NFPA 1976

NFPA 1977

NFPA 1983

NFPA 1991

NFPA 1992

NFPA 1993
Protective Clothing for Structural Fire Fighting

Helmets for Structural Fire Fighting

Gloves for Structural Fire Fighting

Protective Footwear for Structural Fire Fighting

Station/Work Uniforms for Fire Fighters

Protective Clothing for Proximity Fire Fighting

Protective Clothing and Equipment for Wildland Fire Fighting

Fire Service Life Safety Rope, Harness, and Hardware

Vapor-Protective Suits for Hazardous Chemical Emergencies

Liquid Splash-Protective Suits for Hazardous Chemical Emergencies

Support Function Protective Clothing for Hazardous Chemicals Operations

48 National Fire Protection Association. pp. 31, 32. 1995. 


\section{C.8 SAE AMS Textile Specifications ${ }^{49}$}

These specifications for the most part relate to high performance aramid and para-aramid textile materials. They are listed in numerical order.

$3901 \mathrm{~B} \#$

3901/1B \#

3901/2B \#

3901/3B \#

3901/4B \#

3901/5B \#

3901/6B \#

3901/7A \#

3901/8A \#

3901/9A \#

3901/10A \#

3901/11A \#

3901/12A \#

3902B \#
Organic Fiber (Para-Aramid), Yarn and Roving, High Modulus (Oct 92)

Yarn, Organic Fiber (Para-Aramid), High Modulus, OY 390 (2689)/23.5 Tensile Strength, 18 (125)/982 Tensile Modulus, 195 Denier, 0.6\% Finish (Oct 92)

Yarn, Organic Fiber (Para-Aramid), High Modulus, OY 390 (2689)/24.5 Tensile Strength, 17.5 (121)/934 Tensile Modulus, 380 Denier, 0.6\% Finish (Oct 92)

Yarn, Organic Fiber (Para-Aramid), High Modulus, OY 390 (2689)/25.5 Tensile Strength, 16.5 (114)/900 Tensile Modulus, 1140 Denier, 0.6\% Finish (Oct 92)

Yarn, Organic Fiber (Para-Aramid), High Modulus, OY 390 (2689)/24.3 Tensile Strength, 18 (125)/982 Tensile Modulus, 1420 Denier, 0.6\% Finish (Oct 92)

Yarn, Organic Fiber (Para-Aramid), High Modulus, OY 450 (3103)/23.0 Tensile Strength, 17.5 (121)/780 Tensile Modulus, 7100 Denier, 0.6\% Finish (Oct 92)

Yarn, Organic Fiber (Para-Aramid), High Modulus, OY 500 (3447)/23.5 Tensile Strength, 7.5 (121)/800 Tensile Modulus, 4560 Denier, 0.6\% Finish (Oct 92)

Yarn, Organic Fiber (Para-Aramid), High Modulus, OY 390 (2689)/21.5 Tensile Strength, 16.5 (114)/825 Tensile Modulus, 2160 Denier, 0.6\% Finish (Oct 92)

Yarn, Organic Fiber (Para-Aramid), High Modulus, OY 390 (2689)/21.5 Tensile Strength, 18 (124)/982 Tensile Modulus, 195 Denier, 1.2\% Finish (Oct 92)

Yarn, Organic Fiber (Para-Aramid), High Modulus, OY 390 (2689)/24.5 Tensile Strength, 17.5 (121)/934 Tensile Modulus, 380 Denier, $1.2 \%$ Finish (Oct 92)

Yarn, Organic Fiber (Para-Aramid), High Modulus, OY 390 (2689)/23.6 Tensile Strength, 16.5 (114)/885 Tensile Modulus, 1140 Denier, 1.2\% Finish (Oct 92)

Yarn, Organic Fiber (Para-Aramid), High Modulus, OY 390 (2689)/22.2 Tensile Strength, 16.5 (114)/870 Terisile Modulus, 1420 Denier, 1.2\% Finish (Oct 92)

Yarn, Organic Fiber (Para-Aramid), High Modulus, OY 390 (2689)/21.5 Tensile Strength, 16.5 (114)/870 Tensile Modulus, 1420 Denier, 1.2\% Finish (Oct 92)

Cloth, Organic Fiber (Para-Aramid), High Modulus, for Structural Composites (Oct 89)

49 Society of Automotive Engineers, Inc. pp. 101-103. 1994.

\# A previous issue of this document has DODISS acceptance. DODISS adoption means that the document has been coordinated by the tri-services and is approved for military use. 


\section{$3903 \mathrm{~A}+$ \\ $3903 / 1 \mathrm{~A}+$ \\ $3903 / 2 \mathrm{~A}+$ \\ $3903 / 3 \mathrm{~A}+$ \\ $3903 / 4 \mathrm{~A}+$ \\ $3903 / 5 \mathrm{~A}+$ \\ $3903 / 6 \mathrm{~A}+$ \\ $3903 / 7 \mathrm{~A}+$ \\ $3903 / 8 \mathrm{~A}+$}

$3904 \mathrm{~A}$

3904/1A

$3904 / 2 \mathrm{~A}$

3904/3A

3904/4A

3904/5A

$3904 / 6$

$3904 / 7$

$3904 / 8$

$3904 / 9$

$3904 / 10$

$3904 / 11$

$3904 / 12$

3907

$3907 / 1$
Cloth, Organic Fiber (Para-Aramid), High Modulus, Epoxy Resin Impregnated (Oct 85)

Cloth, Organic Fiber, High Modulus, Epoxy Resin Impregnated, OC Style 120, 175 (350) (Jan 88)

Cloth, Organic Fiber, High Modulus, Epoxy Resin Impregnated, OC Style 181, 175 (350) (Jan 88)

Cloth, Organic Fiber, High Modulus, Epoxy Resin Impregnated, OC Style 281, 175 (350) (Jan 88)

Cloth, Organic Fiber, High Modulus, Epoxy Resin Impregnated, OC Style 328, 175 (350) (Jan 88)

Cloth, Organic Fiber, High Modulus, Epoxy Resin Impregnated, OC Style 120, 80 (180) (Jan 88)

Cloth, Organic Fiber, High Modulus, Epoxy Resin Impregnated, OC Style 181, 80 (180) (Jan 88)

Cloth, Organic Fiber, High Modulus, Epoxy Resin Impregnated, OC Style 281, 80 (180) (Jan 88)

Cloth, Organic Fiber, High Modulus, Epoxy Resin Impregnated, OC Style 328, 80 (180) (Jan 88)

Fiber, Organic (Para-Aramid), Yarn and Roving, Intermediate

Modulus, for Cables, Cordage, and Woven Goods (Apr 89)

Yarn, Organic Fiber (Para-Aramid), Intermediate Modulus, 200

Denier, 1.75\% Finish (Apr 89)

Yarn, Organic Fiber (Para-Aramid), Intermediate Modulus, 400

Denier, 1.75\% Finish (Apr 89)

Yarn, Organic Fiber (Para-Aramid), Intermediate Modulus, 1000 Denier, 1.75\% Finish (Apr 89)

Yarn, Organic Fiber (Para-Aramid), Intermediate Modulus, 1000 Denier, 1.5\% Finish, for Weaving (Apr 89)

Yarn, Organic Fiber (Para-Aramid), Intermediate Modulus, 1500 Denier, 1\% Finish, for Cable and Cordage (Apr 89)

Yarn, Organic Fiber (Para-Aramid), Intermediate Modulus, 1500 Denier, 7.0\% Finish, for Cable and Cordage (Apr 89)

Yarn, Organic Fiber (Para-Aramid), Intermediate Modulus, 1500 Denier, Zero Finish, for Cable and Cordage (Apr 89)

Yarn, Organic Fiber (Para-Aramid), Intermediate Modulus, 1500 Denier, 1.0\% Finish, for Weaving (Apr 89)

Yarn, Organic Fiber (Para-Aramid), Intermediate Modulus, 3000 Denier, 0.9\% Finish, for Non-Apparel Ballistic Applications (Apr 89)

Roving, Organic Fiber (Para-Aramid), Intermediate Modulus, 15,000 Denier, 7.0\% Finish, for Cable and Cordage (Apr 89) Roving, Organic Fiber (Para-Aramid), Intermediate Modulus, 15,000 Denier, 1.0\% Finish, for Cable and Cordage (Apr 89) Roving, Organic Fiber (Para-Aramid), Intermediate Modulus, 9000 Denier, 7.0\% Finish, for Cable and Cordage

Cloth, Aramid, Plain and Basket Weave (Oct 85)

Cloth, Aramid, 5 oz per sq. yd $\left(170 \mathrm{~g} / \mathrm{m}^{2}\right)$, Basket Weave (Oct 85)

+ DODISS adpotion means that the document has been coordinated by the tri-services and is approved for military use. 
$3907 / 2$

$3908 \mathrm{~A}$

3909

$3909 / 1$

$3909 / 2$

$3909 / 3$
Cloth, Aramid, 4.3 oz per sq. yd $\left(145 \mathrm{~g} / \mathrm{m}^{2}\right)$, Plain Weave (Oct 85)

Cloth, Aramid (Para), Plain Weave, Thermally Stable (Jan 92) Cloth, Parachute, Aramid, Intermediate Modulus (Jul 85)

Cloth, Parachute, Aramid, $3.0 \mathrm{oz}$ per sq. yd $\left(100 \mathrm{~g} / \mathrm{m}^{2}\right), 350 \mathrm{lb}$ per in. $(61,300 \mathrm{~N} / \mathrm{m})(\mathrm{Jul} \mathrm{85})$

Cloth, Parachute, Aramid, $2.25 \mathrm{oz}$ per sq. yd $\left(75 \mathrm{~g} / \mathrm{m}^{2}\right), 250 \mathrm{lb}$ per in. $(43,800 \mathrm{~N} / \mathrm{m})(\mathrm{Jul} 85)$

Cloth, Parachute, Aramid, $2.0 \mathrm{oz}$ per sq. yd $\left(68 \mathrm{~g} / \mathrm{m}^{2}\right), 230 \mathrm{lb}$ per in. $(40,275 \mathrm{~N} / \mathrm{m})$ and $220 \mathrm{lb}$ per in. $(38,525 \mathrm{~N} / \mathrm{m})(\mathbf{J u l ~ 8 5})$ 
This glossary contains terms common to the fiber, textile, and apparel sectors of the FTA industry, and some terms appearing in the titles of standards listed in Appendix C : FTA Standards Listing. They are listed alphabetically.

\section{alpaca/alpacea}

anthropometry

barre

bast/bass

beam

chambray

chelation

CID

CIM

colorfastness/fastness
1. Animal belonging to the species of Llama; it produces a short textile fiber of 4 inches in one years growth.

2. A thin cloth made of the woolly hair of the alpaca often with dyed silk, cotton, or another fiber in the weft.

The study and technique of human body measurement.

1. A crossover striped cloth with stripes formed by weft from selvage; either woven or printed.

2. A defect due to variation in the number of picks per inch.

Strong woody fibers obtained from the stem, leaves, or fruit of various trees and plants, and known as bast or hard fibers. They are used especially in the manufacture of ropes, cordage, matting, etc.

A cylinder of wood or metal on which the warp from the warping machine is wound before weaving; it is called the yarn beam or weaver's beam, backbeam or section beam.

1. A plain woven cotton or linen fabric with colored warp and white filling that gives a mottled colored surface; used for shirts, children's clothes, and dresses.

2. A similar but heavier carded yarn fabric used for work-shirts and children's play clothes.

The chemical process of forming a ring compound by joining a chelating agent to a metal ion.

Commercial Item Description: The new format for specification of military items, including clothing. It will replace the traditional "MIL-specs." The CID gives the manufacturer more freedom in determining processes and in some cases materials to meet performance criteria.

Computer Integrated Manufacturing: The process of monitoring and controlling manufacturing processes on the shop floor electronically. This requires that machines of different types made by various manufacturers communicate with one another.

Retentive quality of firmness of dyes; such as fastness to light, perspiration, salt water, washing, etc. Fast colors are durable or lasting. (Note that the term, "colorfastness," is referred to in that

\footnotetext{
50 The entries in this glossary were obtained from the following references (some entries have been modified): Link, 1954.

The Riverside Publishing Company, 1984.
} 


\section{colorimeter}

cotton

crocking

crockmeter

degumming/boiling off form and also in the form, "colour fastness," in the standards listings.)

1. An instrument for measuring the depth of color in a liquid by comparison with a standards liquid of the same tint.

2. An instrument or device for determining and specifying colors by reference either to other colors or to certain complex stimuli.

A soft white fibrous substance covering the seeds of various malvaceuous plants. Careful selection has greatly improved the quality and increased the length of the fiber knows as staplelength. On account of its cheapness, cotton is the most important of textile products. The most important property of cotton $s$ the spiral-like appearance or convolution of its fiber which gives it a natural twist, causing the fibers to adhere together while the yarn is being formed. The cotton staple falls into one or more categories in each group:

Table 1 : Cotton Staple Qualities

\begin{tabular}{|c|c|c|c|}
\hline Quality & Color & Feel, Handle & Defects \\
\hline Even & Fair & Soft & Sandy \\
Irregular & Good & Firm & Dusty \\
Good & Spotted & Hard & Leafy \\
Very Good & Stained & Rough & Husky/Howly \\
Strong & Tinged & Towy & Neppy \\
Weak & Highly Colored & & \\
Silky & & & \\
Long Stapled & & & \\
Short Stapled & & & \\
Damaged & & & \\
Coarse & & & \\
& & & \\
\hline
\end{tabular}

In the United States, the cotton receives one of the following overall grades (with "1" being of the highest quality):
1) Middling Fair
2) Strict Good Middling
3) Good Middling
4) Strict Middling
5) Middling
6) Strict Low Middling
7) Low Middling
9) Good Ordinary
8) Strict Good Ordinary

The tendency of excess dye to rub off.

A laboratory device for measuring the fastness of dyes to rubbing.

A process by which the natural gum of silk is dissolved and the released fibers are freed to be drawn. 
desizing

drawing

fiber

\section{filament}

\section{findings}

flax

gabardine
The process of eliminating sizing (stiffening materials) from grey goods preparatory to bleaching, dyeing etc. The sizing substance is first made soluble by an acid or enzyme, then washed out.

1. The process of pulling out or elongating the sliver of the carding machine.

2. Various processes, including giling, reducing, and roving, by which slivers are converted into rovings of the required thickness for spinning.

Any tough substance composed of threadlike tissues and capable of being spun and woven; the minimum length for fibers to be spun into yarn is one-fifth of an inch. Vegetable fibers are yielded by the bast of plants, excepting cotton, which is the hairy tuft of the seed. The following table gives a list of fibers by origin:

Table 2 : Fibers by Origin

\begin{tabular}{|c|c|c|c|}
\hline Animal & Vegetable & Mineral & Synthetic \\
\hline Wool & Cotton & Asbestos & Rayon \\
Hair & Hemp & Metals & Nylon \\
Silk & Flax & & Vinyon \\
Sinew & Jute & & Aralac \\
& Ramio & & Glass \\
& Phormium & & Paper \\
\hline
\end{tabular}

1. A thread or threadlike object, an appendage or a separate fiber; the extreme length of filaments permits their being used in a yarn without twist or with very low twist, and they are usually made into yarn without the spinning operation required for fibers.

2. The single individual unit which is extracted by the silkworm or by the spinneret.

3. Continuous filaments are synthetic and regenerated fibers which have a short staple.

4. Monofilament is a simple filament of sufficient size to function as a yarn in normal textile operation.

5. Multifilament is a rayon yarn with a very large number of fine filaments.

Small, miscellaneous materials used in the apparel manufacture process; not of textile origin; these would include buttons and zippers.

A plant cultivated for its fibers; the long silky bast fiber freed from the stem by retting and various mechanical processes is used in the manufacture of a thread which is woven into a cloth generally known as linen.

A twilled fabric in which warp threads predominate; used as material of clothing for both sexes. 
hand/handle (fabric)

havelock

heald/heddle

hemp

huck/huckabauk

integration

kemp

knitting

medullated (wool)

mercerization

modulus

nep
The reaction to the sense of touch, when raw material or goods are grasped in the hand to judge their quality, taking into account especially their fineness and softness.

A cloth covering for a cap, with a flap to cover and protect the back of the neck.

1. One of the sets of parallel double cords or wires on the loom, which with their mounting compose the harness used to guide the warp.

2. To draw the warp threads with a heddle hook through the heald-eyes or comb, which is a loop formed in each heald.

A plant cultivated for its touch bast fibers, which is obtained similarly to flax; it is used for making cloth and cordage.

A cotton cloth with a rough surface obtained by short floats of warp and weft threads on a plain weave ground texture; employed for towels.

The process of brining all parts of a system or process together and making them compatible.

Thick opaque and wavy fibers with a pointed tip and root, which are shed periodically into the fleece; they develop in nearly all breeds of sheep but principally in mountainous and carpet wool types. They greatly reduce the value of the wool because of the inferior spinning properties; they do not show up dyes.

1. The process of making a fabric by interlacing one or more yarns in a series of connected loops by means of needles, either by hand or by machines; there are rectilinear and circular knitting machines employed to make jerseys, stocking, and the like.

2. Gauge: a standard measure of the fineness of a knitted fabric obtained by counting the number of needles in a given unit of space.

This differs from true kemp because it is not shed but grows with the wool; it is distinguished by the coarser diameter of the medullated cells.

A process to which cotton yarn is subjected to produce luster and shrinkage; the material is treated in a caustic soda solution for one minute and in tension, then neutralized and washed off.

A constant or coefficient that expresses the degree to which a substance possesses some property.

1. Lumps or rolled up and tangled wool fibers which curl up in carding and sometimes also in combing by inefficient setting of the cylinders or rollers; they should be cleared out of the sliver in combing.

2. A cluster of fibers in the wool staple. 
nonwovens

pack

pirn (weft)

roving/roving-frame

saponification

sisal

size/sizing
3. Little knots formed in cotton by immature fibers in the wool staple.

Materials, such as felts, which undergo neither the weaving nor the knitting process. Such fibers may be forced together and the cohesion produced by that process is enough for the intended applications.

1. A bundle or a bale of raw material or of goods; to bale, to load.

2. A measure of scouring wool or wool top weighing $240 \mathrm{lbs}$.

1. A single-headed bobbin or spool in which head and barrel are shaped conically.

2. Yarn wound on the weaver's shuttle.

1. Final product of the drawing process obtained on the roving frames called also dandles, resulting in a strand of wool of the desired thickness for spinning it into a worsted yarn.

2. Drawing process before spinning in worsted yarn manufacture.

1. Chemical process of soap-making.

2. The decomposition of any ester into the corresponding alcohol and fatty acid; also, the similar production of an acid from some other derivative.

3. Saponification number: milligrams of potassium hydride needed to saponify 1.0000 milligrams of the oil, fat, etc., that is being tested.

Approximately 300 species of plants which grow in desert and subtropical regions and supply very strong, smooth, yellowish bast fibers; they are used for upholstery and as substitutes of flax and hemp in the manufacture of sackcloths and carpets.

1. Stiffening or finishing threads, yarn, or fabrics by the use of sizes and glutinous materials; it can be done by means of a sizing apparatus attached to the loom (as in the slasher-sizer) or as a finishing process. There are three types of sizing as follows:

Table 3 : Sizing Types

\begin{tabular}{|l|l|l|}
\hline \multicolumn{1}{|c|}{ Light Sizing } & \multicolumn{1}{|c|}{ Medium Sizing } & \multicolumn{1}{|c|}{ Heavy Sizing } \\
\hline $\begin{array}{l}\text { Up to } 10 \% \text { of size. } \\
\text { Gives a better } \\
\text { handle to the cloth. }\end{array}$ & $\begin{array}{l}\text { From 10\% to 40\% } \\
\text { of size. } \\
\text { Makes the cloth } \\
\text { heavier for jeans } \\
\text { and other clothing. }\end{array}$ & $\begin{array}{l}\text { Up to } 100 \% \text { of } \\
\text { size. } \\
\text { Used for cheap } \\
\text { cotton shirting. } \\
\text { Also starching. }\end{array}$ \\
\hline
\end{tabular}

2. Determination of the count of rovings or yarns.

3. The process of mapping sets of dimensions for a garment to one numerical value. For instance, a size 7 dress denotes particular circumferential and linear measurements. Accurate anthropometric data is crucial to effective sizing. 
slashing

sliver

spinning

stoving

sundries

tannin

tanning

textile

vulcanization

warp/warping

weaving
A process in which sizing is applied to warp threads in their full width; it is used to size the warp yarn with a starch or like substance that will lay all the fibers parallel, and add strength to the yarn; this enables to go through weaving without damage.

A continuous strand of cotton, wool, or other fiber, in a loose untwisted state, produced by a carding, drawing or combing machine.

1. Final drawing of a carded or combed sliver or roving into a yarn inserting the required degree of twists, and winding it upon a cone; this is usually done by ring spinning, the air-jet system, or the open-end centrifugal process.

2. Wool spinning is done by either the woolen or worsted method. 3. Cotton Spinning joins and twists together a series of short fibers to make a thread of desired fineness and length; it consists of the following operations: opening and cleaning the bales, carding or loosening and parallellizing the fibers, drawing the slivers to a uniform length, spinning the slivers into yarn, and winding the yarn from the cones onto spools.

4. Dry spinning is the method for flax, hemp, jute, etc., as well as rayon.

1. The submitting of dampened wool, yarn or cloth to an agent, such as sulfur dioxide fumes, for bleaching.

2. Treating of the silk cocoon by hearing to kill the chrysalis.

Miscellaneous articles used in sewing garments; not of textile origin; these would includes buttons, zippers, etc.

1. Tannic acid.

2. A chemical substance capable of promoting tanning.

The art or process of making leather from rawhides.

Material capable of being spun or woven, knitted, felted, bonded, or crocheted.

A process that increases the strength, resiliency, and freedom from stickiness of a material by combining it with sulfur or other additives in the presence of heat and pressure.

1. A series of threads which are extended lengthwise in the loom and crossed by the weft; it is usually longer and harder twisted than the weft.

2. Warping is the arranging of the chain or series of warp threads according to quality and color, winding them off the bobbins and on to a special beam attached to the loom.

1. The process of interlacing a series of longitudinal yarns with another yarn running crosswise and known as the weft or filling, on machines called looms. 
weaving (con't)

weft
2. A particular pattern or design of weaving such as plain, twill, satin, herringbone, hopsack, etc.

3. Cross weaving is a style of weaving which produces open work effects such as seen in gauze and lenos; it is produced by crossing one warp thread with another, first to one side and then to the other in some definite order.

The thread which is thrown through the warp at right angles by means of a shuttle; it is, as a rule, softer spun and weaker than warp yarn. 


\section{E LIST OF ACRONYMS}

The following is a list of key acronyms used in this paper. Many of them refer to organizations. A brief description and contact information for these organizations can be found in Appendix B: FTA Standards Organizations.

\section{AAMA}

AATCC

ALCA

AMTEX

ANSI

APDES

ARC

ASTM

ATMI

CIM

DAMA

EC

EDI

EDIFACT

FASLINC

FTA

ISO

ITC

NBS

NFPA

NIST

SAE

SAFLINC
American Apparel Manufacturers Association

American Association of Textile Chemists and Colorists

American Leather Chemists Association

American Textile Partnership

American National Standards Institute

Apparel Product Data Exchange Standard

(AAMA) Apparel Research Committee

American Society for Testing and Materials

Apparel Textile Manufacturers Association

Computer-Integrated Manufacturing

(AMTEX) Demand-Activated Manufacturing Architecture

European Community

Electronic Data Interchange

Electronic Data Interchange for Administration, Commerce, and Transport

Fabric and Supplier Linkage Council

Fiber/Textile/Apparel (Industry)

International Organization for Standardization

Integrated Textile Complex

National Bureau of Standards (name changed to NIST in 1988)

National Fire Protection Association

National Institute of Standards and Technology

Society of Automotive Engineers

Sundries and Finding Linkage Council 
SRD

SRM

STEP

TALC

TDI

U CS

VICS

WINS
Standard Reference Data

Standard Reference Material

Standard for the Exchange of Product Model Data

Textile Apparel Linkage Council

Trade Data Interchange

Uniform Communication Standards

Voluntary Interindustry Communication Standard

Warehouse Information Network Standard 


